Computer Science \& Information Technology 

David C. Wyld,

Dhinaharan Nagamalai (Eds)

\section{Computer Science \& Information Technology}

$10^{\text {th }}$ International Conference on Software Engineering and Applications (SEAS 2021), February 20 21, 2021, Dubai, UAE.

AIRCC Publishing Corporation 


\section{Volume Editors}

David C. Wyld,

Southeastern Louisiana University, USA

E-mail: David.Wyld@selu.edu

Dhinaharan Nagamalai (Eds),

Wireilla Net Solutions, Australia

E-mail: dhinthia@yahoo.com

ISSN: $2231-5403$

ISBN: 978-1-925953-36-7

DOI: $\quad$ 10.5121/csit.2021.110201- 10.5121/csit.2021.110205

This work is subject to copyright. All rights are reserved, whether whole or part of the material is concerned, specifically the rights of translation, reprinting, re-use of illustrations, recitation, broadcasting, reproduction on microfilms or in any other way, and storage in data banks. Duplication of this publication or parts thereof is permitted only under the provisions of the International Copyright Law and permission for use must always be obtained from Academy \& Industry Research Collaboration Center. Violations a reliable to prosecution under the International Copyright Law.

Typesetting: Camera-ready by author, data conversion by NnN Net Solutions Private Ltd., Chennai, India 


\section{Preface}

The $10^{\text {th }}$ International Conference on Software Engineering and Applications (SEAS 2021), February 20 21, 2021, Dubai, UAE and $10^{\text {th }}$ International Conference on Control, Modelling, Computing and Applications (CMCA 2021) and 8th International Conference on Advanced Computing (ADCO 2021) was collocated with $10^{\text {th }}$ International Conference on Software Engineering and Applications (SEAS 2021). The conferences attracted many local and international delegates, presenting a balanced mixture of intellect from the East and from the West.

The goal of this conference series is to bring together researchers and practitioners from academia and industry to focus on understanding computer science and information technology and to establish new collaborations in these areas. Authors are invited to contribute to the conference by submitting articles that illustrate research results, projects, survey work and industrial experiences describing significant advances in all areas of computer science and information technology.

The SEAS 2021, CMCA 2021 and ADCO 2021 Committees rigorously invited submissions for many months from researchers, scientists, engineers, students and practitioners related to the relevant themes and tracks of the workshop. This effort guaranteed submissions from an unparalleled number of internationally recognized top-level researchers. All the submissions underwent a strenuous peer review process which comprised expert reviewers. These reviewers were selected from a talented pool of Technical Committee members and external reviewers on the basis of their expertise. The papers were then reviewed based on their contributions, technical content, originality and clarity. The entire process, which includes the submission, review and acceptance processes, was done electronically.

In closing, SEAS 2021, CMCA 2021 and ADCO 2021 brought together researchers, scientists, engineers, students and practitioners to exchange and share their experiences, new ideas and research results in all aspects of the main workshop themes and tracks, and to discuss the practical challenges encountered and the solutions adopted. The book is organized as a collection of papers from the SEAS 2021, CMCA 2021 and ADCO 2021.

We would like to thank the General and Program Chairs, organization staff, the members of the Technical Program Committees and external reviewers for their excellent and tireless work. We sincerely wish that all attendees benefited scientifically from the conference and wish them every success in their research. It is the humble wish of the conference organizers that the professional dialogue among the researchers, scientists, engineers, students and educators continues beyond the event and that the friendships and collaborations forged will linger and prosper for many years to come.

David C. Wyld, Dhinaharan Nagamalai (Eds) 


\section{General Chair}

David C. Wyld,

Dhinaharan Nagamalai(Eds)

\section{Program Committee Members}

Abdessamad Belangour, Abdolreza Hatamlou, Adriana Carla, Afaq Ahmad, Ahmad Fakharian, Ahmad Khasawneh, Ahmed A. Elngar, Ahmed Korichi, Ajay Anil Gurjar, Akhil Gupta,

Ali Asghar Rahmani Hosseinabadi, Amal Azeroual,

Amel Ourici,

Anas M.R. Al Sobeh, Anirban Banik, Ansar Daghouri, Arianit Maraj, Asif Irshad Khan, Aubrun, Ayad Salhieh, Benaddy Mohamed, Berenguel,

Blasco,

Bogdan,

Bokor,

Bouhorma Mohammed,

Boukari Nassim,

Bucher,

Camacho,

Celia Ghedini Ralha,

Chien-Cheng $\mathrm{Yu}$,

Ching-Nung Yang,

Claudio Schifanella,

Dariusz Jakóbczak,

Dhanya Jothimani,

Dimitris Kanellopoulos,

Ding Wang,

Djamel Eddine,

Elhabil Brahim,

El-Sayed M. El-Horbaty,

Emad Awada,

Emma Moupojou,

\section{Organization}

Jackson State University, USA

Wireilla Net Solutions, Australia
University Hassan Ii Casablanca, Morocco

Islamic Azad University, Iran

Federal University of Campina Grande (UFCG), Brazil

Sultan Qaboos University, Oman

Islamic Azad University, Iran

Irbid National University, Jordan

Beni-Suef University, Egypt

University Of Ouargla, Algeria

Sipna College of Engineering \& Technology, India

Lovely Professional University, India

Islamic Azad University, Iran

Mohammed V University, Morocco

University Badji Mokhtar Annaba, Algeria

Yarmouk University, Jordan

National Institute of Technology Agartala, India

University Hassan Ii of Casablanca, Morocco

Aab College, Republic Of Kosovo

King Abdulaziz University, Saudi Arabia

Christophe University of Lorraine, France

Australian College of Kuwait (Ack), Kuwait

Ibn Zohr University, Morocco

Manuel Universidad de Almeria, Spain

Xavier Universitat Politécnica de Valencia, Spain

Stjepan Univ. of Zagreb, Croatia

Jozsef Hungarian Academic of Sciences, Hungary

Fstt, Morocco

Skikda University, Algeria

Roberto Scuola Univ. Professionale, Switzerland

Eduardo F. Universidad de Sevilla, Spain

University of Brasalia (Unb), Brazil

Hsiuping University of Science and Technology, Taiwan

National Dong Hwa University, Taiwan

University Of Turin, Italy

Koszalin University of Technology, Poland

Ryerson University, Canada

University of Patras, Greece

Nankai University, China

University Of Biskra, Algeria

Ibn Zohr University, Morocco

Ain Shams University, Egypt

Applied Science University, Jordan

University Of Yaounde, Cameroon 
Erdal Ozdogan,

Esmaiel Nojavani,

Faeq A.A.Radwan,

Fazlollah Abbasi,

Fei Hui,

Felix J. Garcia Clemente,

Froilan Mobo,

Gajendra Sharma,

Gurkana,

Gururaj H L,

Haider M. Alsabbagh,

Haitham J.Taha,

Hamid Alasadi,

Hamid Ali Abed AL-Asadi,

Hayet Mouss,

Hongzhi,

Iyad Alazzam,

Jalel Akaichi,

Kamel Hussein Rahouma,

Kenjiro T. Miura,

Mahdi Abbasi,

Mallikharjuna Rao K,

Merniz Salah,

Metasebia Alemante,

Mohammad Al_Selam,

Mohammed Bouhorma,

Muhammad Sarfraz,

Nalini Chidambaram,

Narasimham Challa,

Neda Darvish,

Osama Rababah,

P.V.Siva Kumar,

Picky Butani,

Rafael Valencia Garcia,

Rami Raba,

Reza Ebrahimi Atani,

S. M. Saniul Islam Sani,

S.Sridhar,

Saad Aljanabi,

Said Nouh,

Saif aldeen Saad Obayes,

Seema Verma,

Sikandar Ali,

Smain Femmam,

Sreenivasa Reddy E,

Sudarshan Patel,

Tranos Zuva,

Tripathy B K,

Venkateswara Rao,

Wenyuan Zhang,

Xiaodong Liu,

Yu-Sheng Lu,
Gazi University, Turkey

University Of Isfahan, Iran

Near East University, Turkey

Islamic Azad University, Iran

Chang'an University, P.R.China

University Of Murcia, Spain

Merchant Marine Academy, Philippines

Kathmandu University, Nepal

Yildiz Technical University, Turkey

Vidyavardhaka College of Engineering, India

University Of Basrah, Iraq

University Of Technology, Iraq

Basra University, Iraq

Iraq University College, Iraq

Batna Univeristy, Algeria

Harbin Institute of Technology, China

Yarmouk University, Jordan

University of Tunis, Tunisia

Minia University, Egypt

Shizuoka University, Japan

Bu-Ali Sina University, Iran

VIT-AP University, India

University of Constantine 2, Algeria

ZTE University, China

University of Technology, Iraq

FST of Tangier, Morocco

Kuwait University, Kuwait

Bharath University, India

SR Engineering College, India

Islamic Azad University, Iran

University of Jordan, Jordan

VNR VJIET, India

Savannah River National Laboratory, US

University of Murcia, Spain

Al Azhar University, Palestine

University of Guilan, Iran

System \& Network, Bangladesh

Easwari Engineering College, India

Computer Technology Engineering, Iraq

Hassan II university of Casablanca, Morocco

Shiite Endowment Office, Iraq

Banasthali University, India

China University of Petroleum-Beijing, China

UHA University, France

Acharya nagarjuna university, India

Gujarat Technological University, India

Tshwane University of Technology, India

VIT University, India

CVR College of Engineering, India

Tianjin University, China

Edinburgh Napier University, UK

National Taiwan Normal University, Taiwan 


\section{Technically Sponsored by}

Computer Science \& Information Technology Community (CSITC)

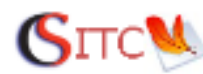

Artificial Intelligence Community (AIC)

Soft Computing Community (SCC)

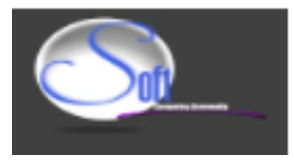

Digital Signal \& Image Processing Community (DSIPC)

Organized By

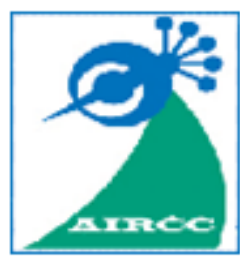

Academy \& Industry Research Collaboration Center (AIRCC) 


\section{TABLE OF CONTENTS}

\section{$10^{\text {th }}$ International Conference on Software Engineering and Applications (SEAS 2021)}

Validation Method to Improve Behavioral Flows on UML Requirements Analysis Model by Cross-Checking with State Transition Model $01-15$ Hikaru Morita and Saeko Matsuura

Intelligent System for Solving Problems of Veterinary Medicine on the Example of Dairy Farms.

Shopagulov Olzhas, Tretyakov Igor and Ismailova Aisulu

\section{$10^{\text {th }}$ International Conference on Control, Modelling, Computing and Applications (CMCA 2021)}

Lossless Steganography on Orthogonal Vector for 3D H.264 with Limited Distortion Diffusion. $35-52$

Juan Zhao and Zhitang Li

Reversible Data Hiding based on Two-dimensional Histogram Shifting. $.53-69$

Juan Zhao and Zhitang Li

\section{$8^{\text {th }}$ International Conference on Advanced Computing (ADCO 2021)}

Cell Switches Model Applying Markov Chain Stochastic Model Check on Between Two Population with Regards to MRNA and Proteins and Neurons both Classically and Quantum Computationally. $.71-95$

Qin He, Rubin Wang and Xiaochuan Pan 


\title{
VALIDATION METHOD TO IMPROVE BEHAVIORAL FLOWS ON UML REQUIREMENTS ANALYSIS MODEL BY CROSS- CHECKING WITH STATE TRANSITION MODEL
}

\author{
Hikaru Morita and Saeko Matsuura \\ Graduate School of Engineering and Science, Shibaura Institute of Technology, \\ Minuma-ku 307, Saitama, Japan
}

\begin{abstract}
We propose a method to evaluate and improve the validity of required specifications by comparing models from different viewpoints. Inconsistencies are automatically extracted from the model in which the analyst defines the service procedure based on the initial requirement; thereafter, the analyst automatically compares it with a state transition model from the same initial requirement that has been created by an evaluator who is different from the analyst. The identified inconsistencies are reported to the analyst to enable the improvement of the required specifications. We develop a tool for extraction and comparison and then discuss its effectiveness by applying the method to a requirements specification example.
\end{abstract}

\section{KEYWORDS}

Requirements Specification, UML Modeling, Validation, Behavior Model.

\section{INTRODUCTION}

In recent years, a system that provides services is often complex, and it is linked with various hardware and other systems. To build a system that satisfies the final service goal, it is important to verify that the requirement specifications satisfy the goal after considering the characteristics of the system components in the requirements analysis phase. We studied model driven development, which defines requirements specifications using unified modeling language (UML) models based on use case analysis [1]. Further, we verified the inconsistencies between the models using the model-verification technique [2,3] and converted the investigated specifications into products at the design and implementation phases [4]. Similar to our approach [2], Tariq et al. [5], and Rafe et al. [6] transformed the activity diagram created in the requirements analysis stage into a finite state model suitable for model verification tools, i.e., a formal verification technique. Hence, exhaustive verification was performed to guarantee reachability and safety. However, although these verification methods can confirm the validity of the service procedure described, any excess or deficiency in the user request cannot be verified. To determine the excess or deficiency in a requirements specification, the requirements specification must be interpreted from multiple perspectives; in addition, requirements that are necessitated must be identified based on their differences. 
Herein, we compare a requirements analysis model by defining the service procedure based on the consensus of multiple developers and the state transition model from the viewpoint of the state that the system should assume to perform the service. The purpose is to cross-validate the requirements analysis model and determine the excess or deficiency of the behavior.

The remainder of this paper is organized as follows. Section 2 describes the method to define a UML requirements analysis model and the role of the state transition model for evaluating the behavioral model. Section 3 describes the comparison method for the extracted and evaluation models. Section 4 discusses the effectiveness of our method using a case study. Finally, Section 5 discusses the conclusions and directions for future research.

\section{REQuirements ANAlysis Model AND Evaluation Model}

The service can be realized by linking the use cases provided by the system. In recent complex systems, the boundaries of each use case and the method to link the use cases at the early stage of development must be identified. We focused on exchanging information at each boundary in a system workflow and defined the cooperation and behavior of subsystems based on the procedure by the action flow using an activity diagram.

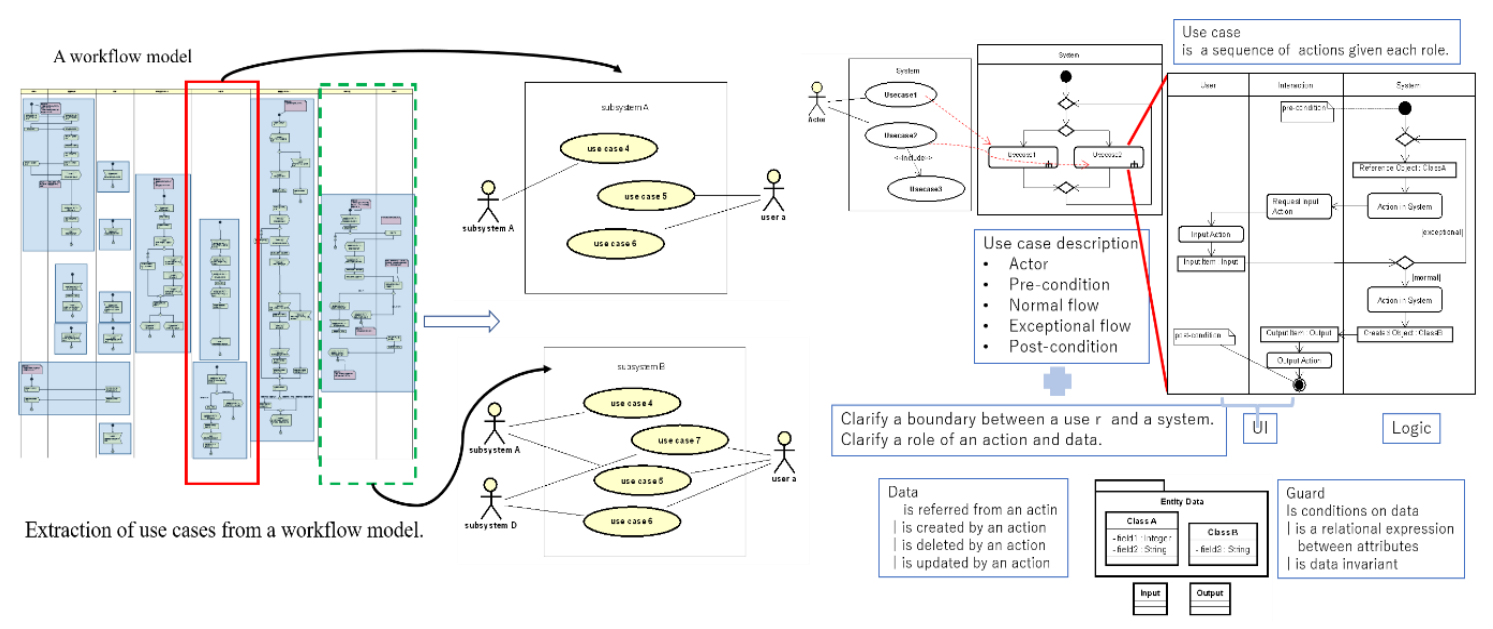

Figure 1: UML Requirements Analysis Model

A workflow is beneficial for realizing a service that utilizes the use cases of each subsystem. To achieve the system goals, the data exchange that is required to satisfy the service goals at each subsystem boundary must be clarified. Each partition in the activity diagram represents each subsystem and the users, and a set of use cases of each subsystem is described within the partition. Consequently, the cooperation between all users and subsystems is clarified, and the service procedure of the system is correctly defined.

Figure 1 shows the requirements analysis process after validating the workflow model. The use case diagram of each subsystem is described based on the partition of each subsystem of the workflow. At this point, the subsystem and the user who are exchanging data with the subsystem become actors in the use case diagram. Each use case not only defines the behavior by the activity diagram, but it also clarifies the behavior related to the data defined in the class diagram in Figure 1. We name this model the UML requirements analysis model. In such behavioral modeling, the procedure of the required function can be easily understood from the control structure. However, as the change in the state of the system due to the action is unclear, it is difficult to determine all the states that the system should assume. 
Meanwhile, the state transition model defines the behavior by changing the state of the system by external or internal events. It is easy to understand whether the system requirements are comprehensively analyzed by specifying the state name; however, it is difficult to confirm the execution procedure of the system function and its relationship with other subsystems. Therefore, activity diagrams are suitable for defining the entire system workflow, which includes coordination between subsystems. On the contrary, the state transition model organizes the requirements that the subsystem should satisfy based on the state that the system should assume. Therefore, it can be a test case of requirements specification defined by the former; as such, we propose using it as the evaluation model of the requirements analysis model, as shown in Figure 2.

\section{Requirements Analysis Model}

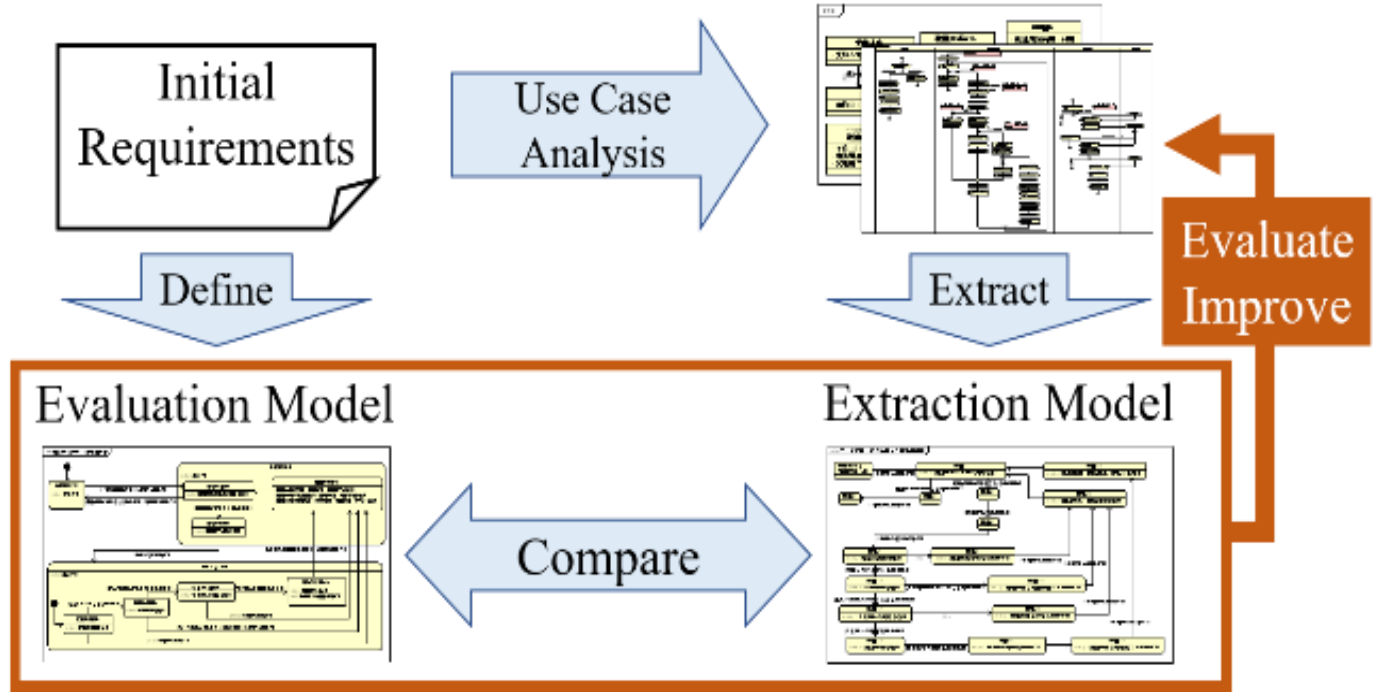

Figure 2: Validation Method by Comparing Extracted Model with Evaluation Model.

\section{CONFIRMation Method of ReQuirements Specification s Using State Transition MOdel}

\subsection{Extraction of State Transition Model from Workflow}

The state was extracted from the requirements analysis model by focusing on the workflow control structure and actions. The extracted state transition model is named as the extraction model.

Table 1: Extraction Rules

\begin{tabular}{|c|c|c|c|}
\hline $\begin{array}{l}\text { Activity model in } \\
\text { workflow }\end{array}$ & State transition model & $\begin{array}{l}\text { Activity model in } \\
\text { workflow }\end{array}$ & $\begin{array}{l}\text { State transition } \\
\text { model }\end{array}$ \\
\hline action & stateo & artiono & stateon \\
\hline [guardo] \& [auard] & [and of & $\frac{1}{\text { acciont }}$ & taction \\
\hline$\frac{v}{\text { actor } 17}$ & state- & $\frac{1}{\text { action } 2}$ & state \\
\hline
\end{tabular}




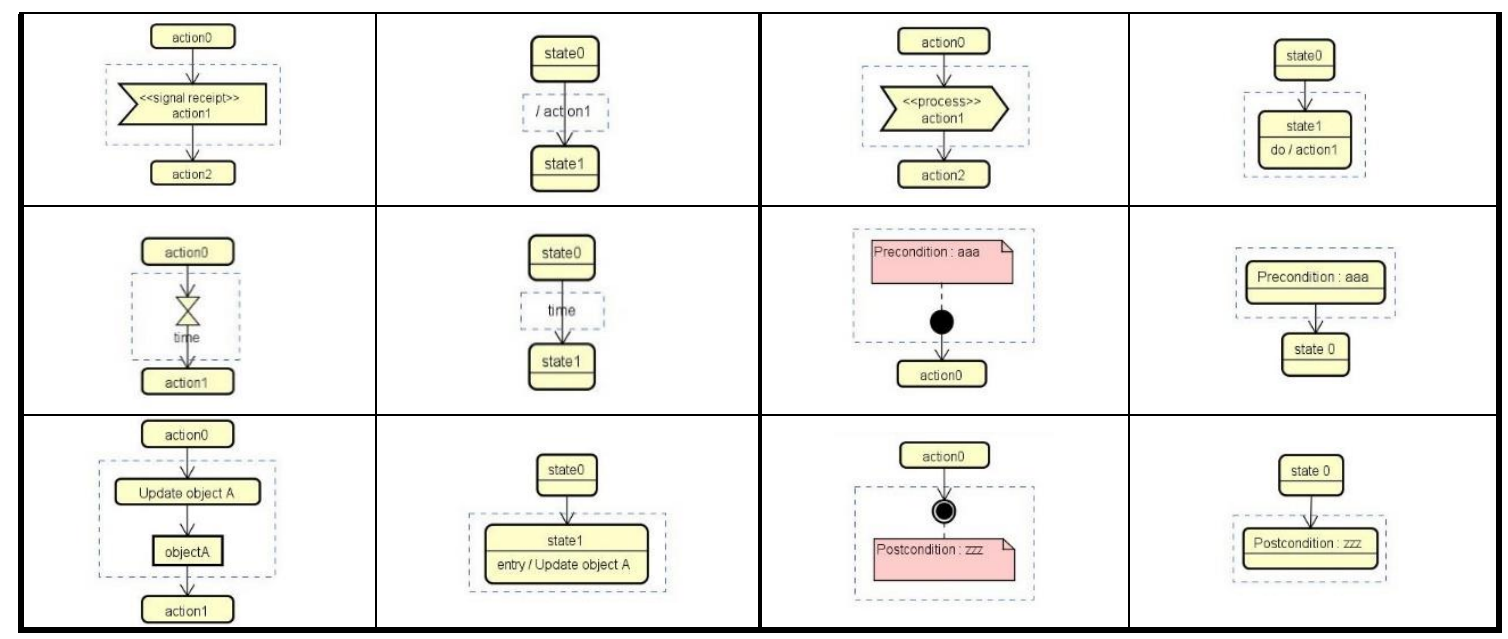

Table 1 shows the conversion rule from the requirements analysis model to the state transition model based on the following reason. First, the decision merge node is a transition control node and the transition differs depending on the branch condition; therefore, the separation of the states before and after that is identifiable.

Next, we focus on the action nodes. This is because the control structure divides the state by its guard, and it is assumed that the state changes by executing the action. Such actions comprise actions that receive data from other systems, actions that indicate the passage of time, actions that change the attributes of the system, and actions that are unique to the subsystems. Actions that receive data from other systems are described as signal receiving nodes, whereas actions that indicate the passage of time are described as timers. Signal-receiving nodes and timers are converted into events in the state machine diagram, signal sending nodes, and update actions, wherein the attributes of an object are converted into entry actions in a state; meanwhile, other actions are converted into actions on a transition arrow. Because the pre- and post-conditions directly represent the state of the system, they are used as the state of the state transition model. Figure 4 shows an example of the extraction model, which is expressed as a one-layer state transition model under the extraction rules. This extraction model is generated based on the rules in Table 1 from the activity diagram shown in Figure 3. 


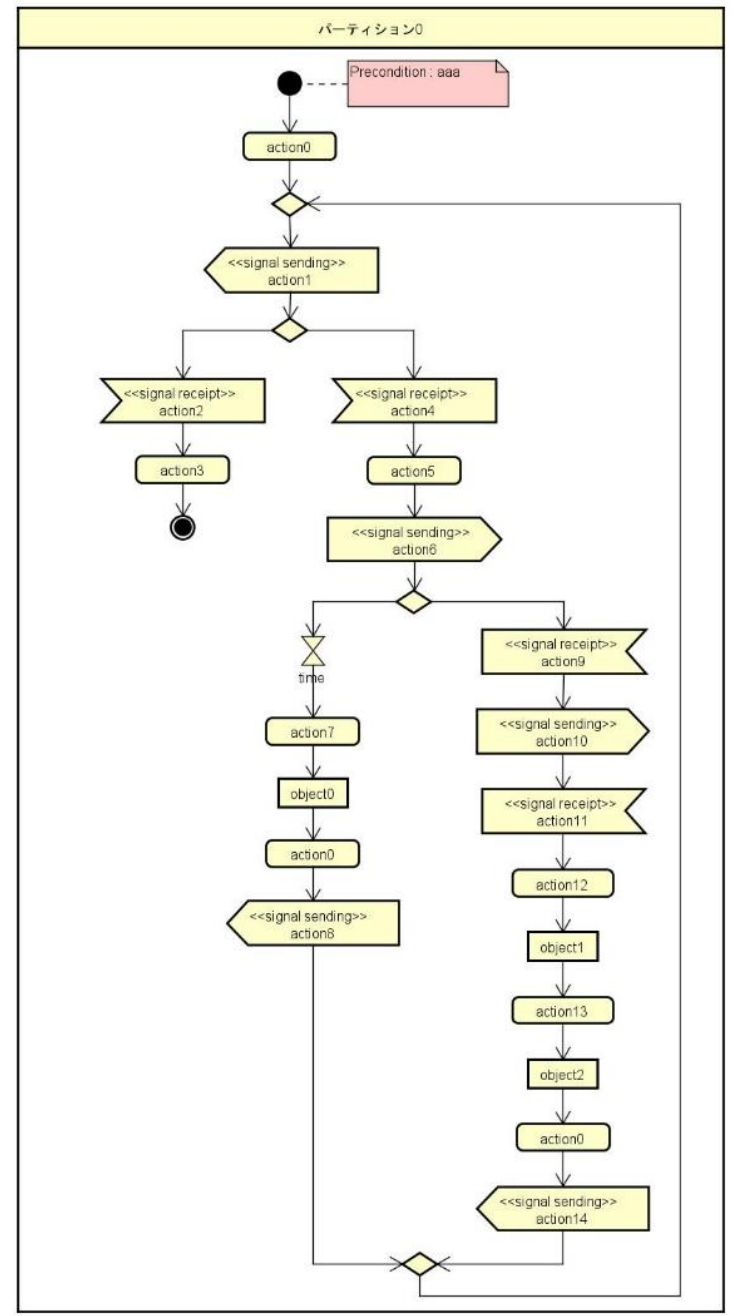

Figure 3: Example of Activity Model

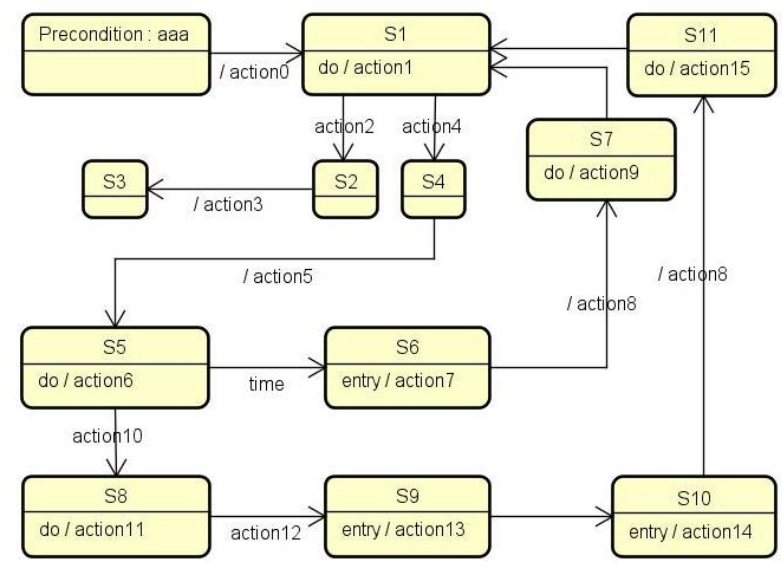

Figure 4: Example of Extraction Model

To confirm whether the extraction model includes the states to be assumed by the system indicated by the evaluation model, the states to be assumed by the system must be described 
appropriately based on the characteristics of the target system in the evaluation model. Because the workflow defines the cooperation scenario between subsystems, the state to be assumed by the subsystem should first be determined based on the state of each partner to be linked, as well as the type of work scenario to be executed within that state.

Consequently, the state of the evaluation model is defined by dividing it into layers for each viewpoint, as shown in Figure 5. In addition, if the words and phrases described in the evaluation model are freely described by the evaluator, it will be difficult to compare the contents of the state transition model. Therefore, we herein provide a list of actions of the defined workflow to unify words and phrases.

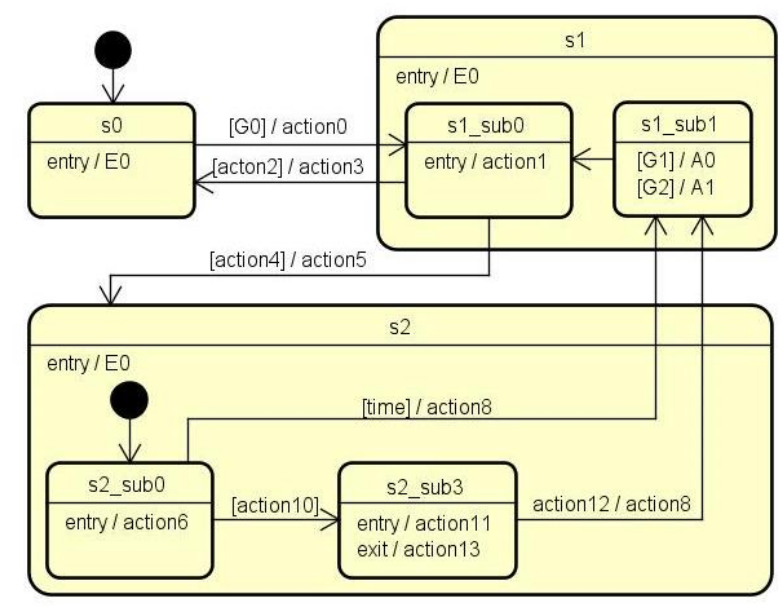

Figure 5: Example of Evaluation Model

\subsection{Validation Process by Comparing Extraction and Evaluation Models}

In the extraction model, the states are subdivided into one hierarchy based on the action and branch structure; therefore, each state is divided into the hierarchical states of the evaluation model based on a list of common actions prepared in advance. Table 2 provides a judgment list of behavior of the evaluation model shown in Figure 5.

Table 2: Judgment List of Behavior of Evaluation Model Shown in Figure 5

\begin{tabular}{|c|c|c|}
\hline s0 & s1 & s2 \\
\hline E0 & E0 & E0 \\
\hline G0 & action1 & action6 \\
\hline & action2 & time \\
& action3 & action0 \\
& action4 & action9 \\
\hline & action5 & action10 \\
& G1 & action12 \\
& A0 & action11 \\
\hline & G2 & action0 \\
\hline
\end{tabular}

This list was generated for each hierarchy to be compared. All states, actions, and state transition contents, such as events, guards, and actions described in the state to be classified were acquired. Because the actions included in each state $s_{i}$ and the event guard actions described in the state 
transitions whose transition source $\mathrm{s}_{\mathrm{i}}$ occur in state $\mathrm{s}_{\mathrm{i}}$, they are classified into $\mathrm{s}_{\mathrm{i}}$. Duplicated labels listed in Table I are in bold font.

State transitions that transition between classification targets $\left(s_{i}, i=1, \ldots n\right)$ are beneficial for classifying hierarchies. We name these transitions "external transitions."

Subsequently, the difference in interpretation between the two models is identified stepwise from the following viewpoints.

Step 0: The name of the state in the extraction model is compared with the name of each state in the evaluation model.

As shown by the rules listed in Table 1, the pre- and post-conditions in the workflow directly represent the states; therefore, they are compared with the state names of the extraction model obtained. Furthermore, by comparing between Figures 4 and 5, whether aaa matches any of the states can be determined.

Step 1: The state of the extraction model is classified based on the operation described in the state.

For each state in the extraction model, some behaviors included in it are verified if they can be classified into the state in the evaluation model using the judgment list. The category of the evaluation model that includes the state in which the behavior is described can be identified among the states of the extracted model. If the behavior is not described and the description element of the extraction model is not unique in the judgment list, then, the classification cannot be specified; consequently, it is set as unknown.

\section{Step 2: Classify by the event, guard, and action described in the state transition}

For an unknown state that cannot be classified by its own behavior, the state transition that exits from that state is acquired. The unknown state is classified by the event, guard, and action described in the acquired state transition using the judgment list. If no transition occurs, then, the description element is not unique in the judgment list, or no description element (we name it an unconditional transition) exists; consequently, the classification cannot be specified and hence, it is set as unknown. Table 3 lists the categories following the above mentioned three steps, and states divided by the first step are expressed in bold font.

Table 3: Classification by Steps 0,1, and 2

\begin{tabular}{|c|c|c|c|}
\hline s0 & s1 & s2 & unknown \\
\hline Precondition : aaa & s1 & s5 & s3 \\
\hline & s2 & s8 & s6 \\
& s4 & s9 & s7 \\
& & & $s 10$ \\
\hline & & & s11 \\
\hline
\end{tabular}

Step 3: Classify using an external transition based on the state before and after the state transition

For the unknown state that has not yet been classified after the classification in the state transition, the previous state is acquired from the connected state transition. If the acquired state has already been classified into the state of the evaluation model, it is then classified into the 
same state. If it cannot be classified, the previous state is acquired from the state transition associated with the previous state, and the same judgment is performed. The acquisition of the previous state continues unless the behaviors on the connected state transition are the last in the list of behaviors of the abovementioned external transition. This is because the last behavior of the external transition divides the states in the same layer.

Table 4: Result of Step 3

\begin{tabular}{|c|c|c|c|}
\hline s0 & s1 & s2 & unknown \\
\hline Precondition : aaa & s1 & s5 & \\
\hline$\underline{s 3}$ & s2 & s8 & \\
& s4 & s9 & \\
& s7 & s6 & \\
& s11 & s10 & \\
\hline
\end{tabular}

Table 4 lists the results of the extraction model divided into the first layer of the evaluation model.

After this step, the extraction model is divided into the first layer in the evaluation model such that the number of states in this layer can be compared. Considering this difference, we can identify the following problems:

A) Among the states of the extraction model, a state exists that cannot be classified.

Therefore, the corresponding action of the workflow is not described in the evaluation model; hence, it may be in an unnecessary state that is derived from some excess behaviors. As the action flow causing the workflow defect is identified by tracing back the extraction rule, the flow can be rectified.

B) The extraction model does not contain a state that corresponds to the state of the evaluation model.

Therefore, the state considered in the evaluation model is not described as an action that can identify the state, such as a workflow signal reception action or an action for data. Some new actions must be added to identify the state of the workflow as the required state or flow may be missing.

C) The extracted model does not contain a state name that includes the state name of the evaluation model.

Therefore, the pre- and post-conditions may not be described in the workflow or the appropriate condition may not be defined. Hence, a note is added to the initial and final nodes of the workflow to add the pre- or post-condition.

After modifying the workflow or the evaluation model such that the number of states is equivalent, the number of state transitions of the modified extraction and evaluation models are compared.

The difference in the number of transitions is compared by generating a state transition table for both models. If the numbers differ, then the behaviors of the transitions are compared to identify the transitions that can be considered the same. The remaining transitions in the target state including some internal state transitions must be compared to determine whether their combinations are the same. In this case, the workflow transition condition may be ambiguous; 
therefore, some actions must be added. Moreover, if an additional transition exists, whether the behavior is necessary must be considered.

For all layers of the evaluation model, the previous steps are repeated to improve the workflow based on the observed difference. The improved extraction and evaluation models become a state transition model in which both the states and transitions are equal.

Figure 6 shows an extraction model after the abovementioned classification for all layers.

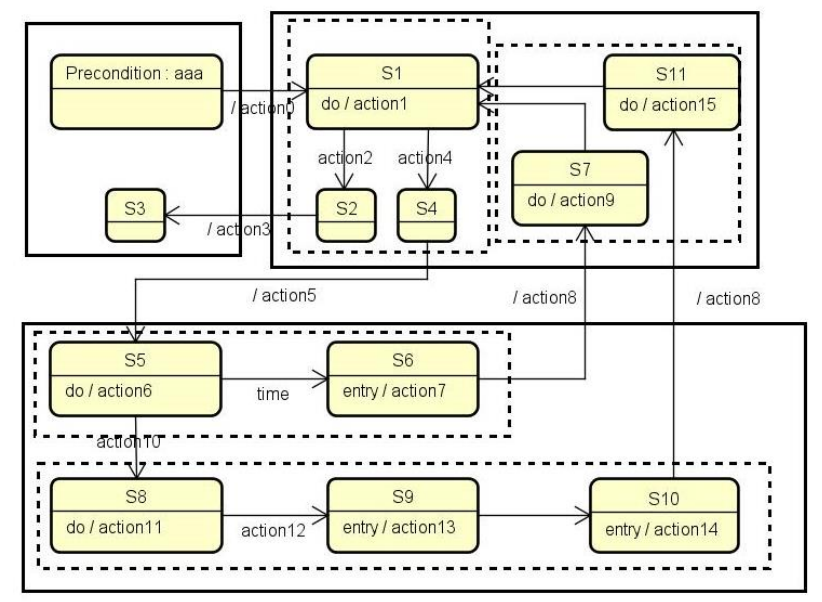

Figure 6: Classified Extraction Model

Finally, the correspondence of each behavior is verified, and the excess or deficiency of the behavior is determined. The difference between the two types of behavior models is noteworthy. In the behavior model based on the activity diagram, when the state changes by the change in the attribute of an object, it is defined by an action that changes the state. However, in the state transition model, it is typically expressed directly by the state name. Therefore, the meanings of the different expressions must be confirmed when comparing the descriptive elements in both models. In the correspondence between the evaluation model shown in Figure 5 and the classified extraction model shown in Figure 6, action7 and action14 in the extraction model can be interpreted as corresponding to G1 and G2 of the evaluation model, respectively.

By comparing the description elements, we can perform the following modifications:

- Rectify the behavioral expressions of action9 and action 15 to A0 and A1, respectively.

- Because G0 is omitted in the extraction model, it is added to the workflow preconditions.

- Because behavior E0 is required in all states in the evaluation model, it should be a precondition in the workflow, or the behavior at the start of each state as an action should be added.

\section{Case Study and Discussion}

This method was applied to the automatic baggage transport system, which is a PBL task in our department. This system is a parcel delivery system that links two autonomous vehicle-type robots with six subsystems: a relay station, headquarters, a reception desk, and the recipient's house. Figure 7 shows the circumstances and the relation between all subsystems. 


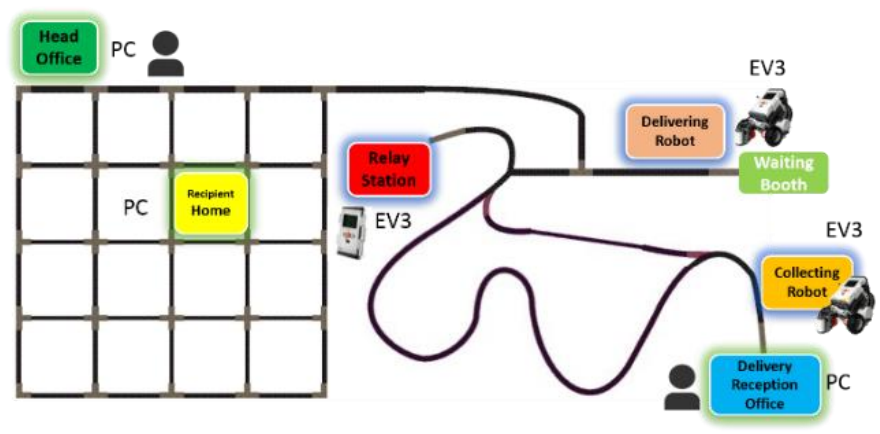

Figure 7: Circumstances and Relation between Sub systems.

First, the workflow of this system is defined as a cooperation model of six subsystems. Figure 8 shows the extraction model generated from the workflow of the relay station based on the extraction rules. Figure 9 is an evaluation model that defines the requirements of the relay station as a state transition model. The orange box in Figure 8 shows the classification results based on the process outlined in Section 3 for the evaluation model. In this case, as a result of the state classification, the number of states in the first layer is equivalent to that in the evaluation model. However, as shown in Figures 7 and 8, the number of transitions between "waiting" to "working with delivery robot" differs. Comparing the state transitions inside both states, it was discovered that the difference in the transition branching is caused by the part surrounded by the dotted line in Figure 9.

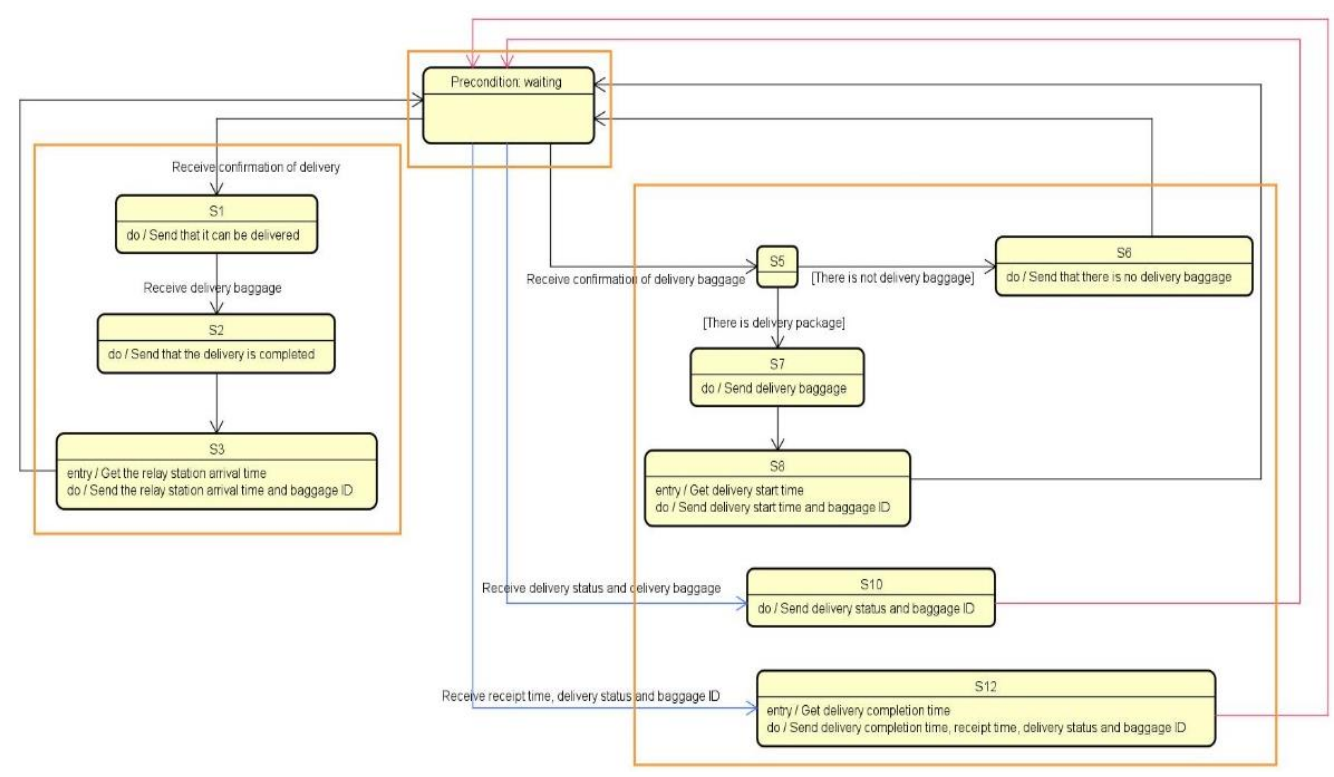

Figure 8: Extraction Model after Steps 0, 1, and 2 


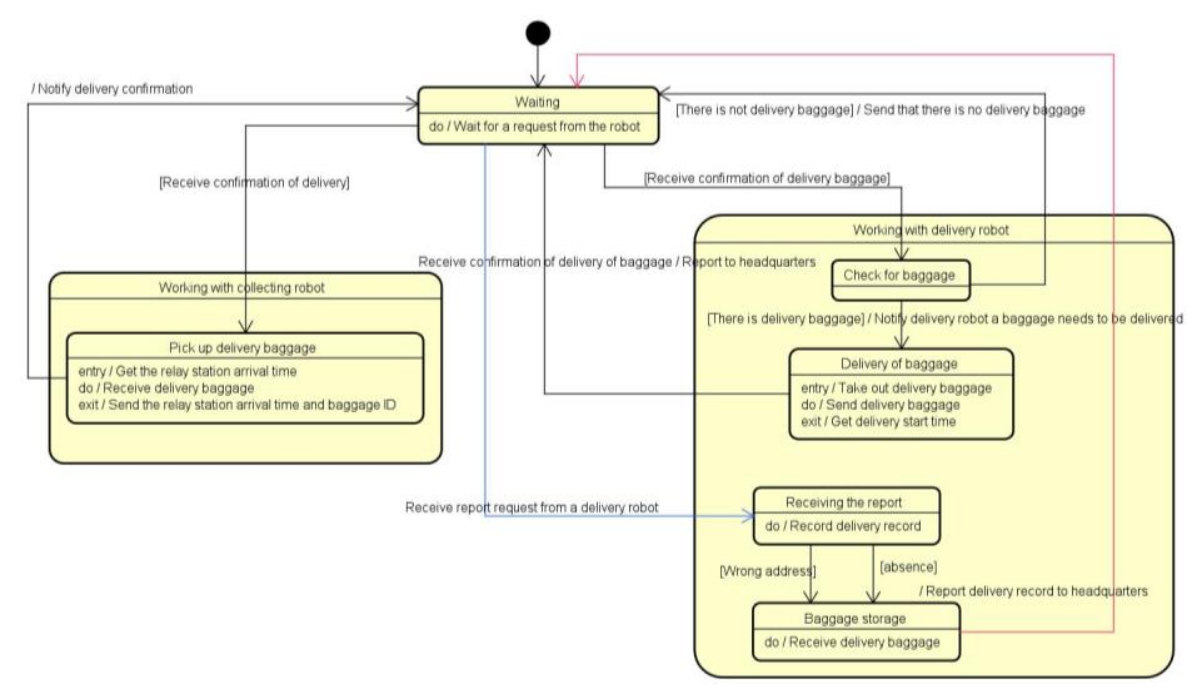

Figure 9: Evaluation Model

Comparing the state transitions inside both states, it is clear that the black transitions have the same meaning. However, because the remaining two transitions (see two sets of red and blue lines in Figure 8) have different transition branches depending on the part surrounded by the dotted line in Figure 9, a difference in meaning is generated.

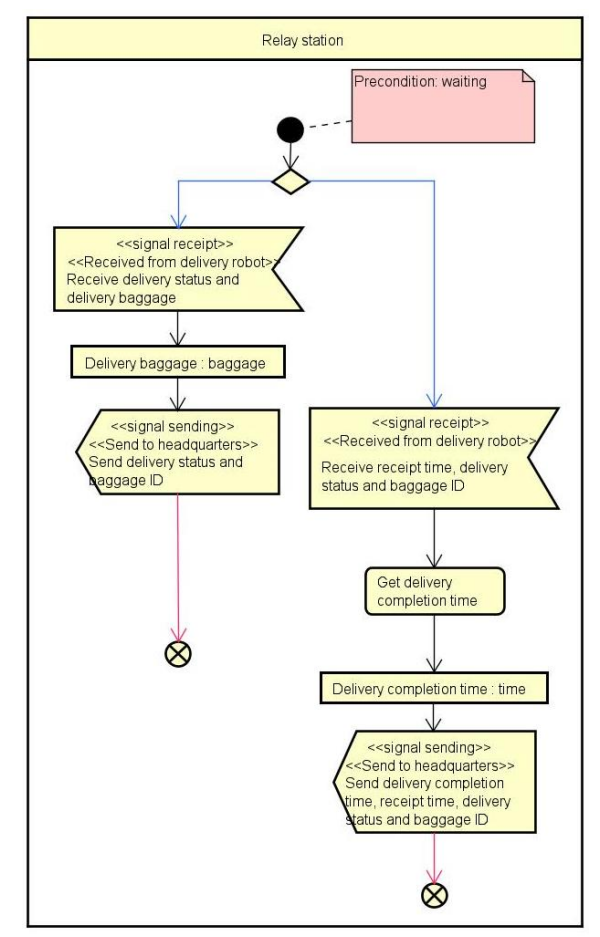

Figure 10: Corresponding Part of Workflow

Figure 10 shows a part of the workflow corresponding to the two blue lines in the extraction model. This can be automatically identified from states S10 and S12 based on the conversion rules. 
Reviewing the workflow, it was discovered that the branching condition can be read from the branching processing flow, but the branching condition based on the delivery status value specified in the evaluation model was not specified in the workflow. Because the design model for the final program will be derived from the workflow, the processing procedure should be described in clear terms at this stage. Therefore, the workflow was modified as shown in Figure 11. Figure 12 shows the extraction model regenerated from the modified workflow.

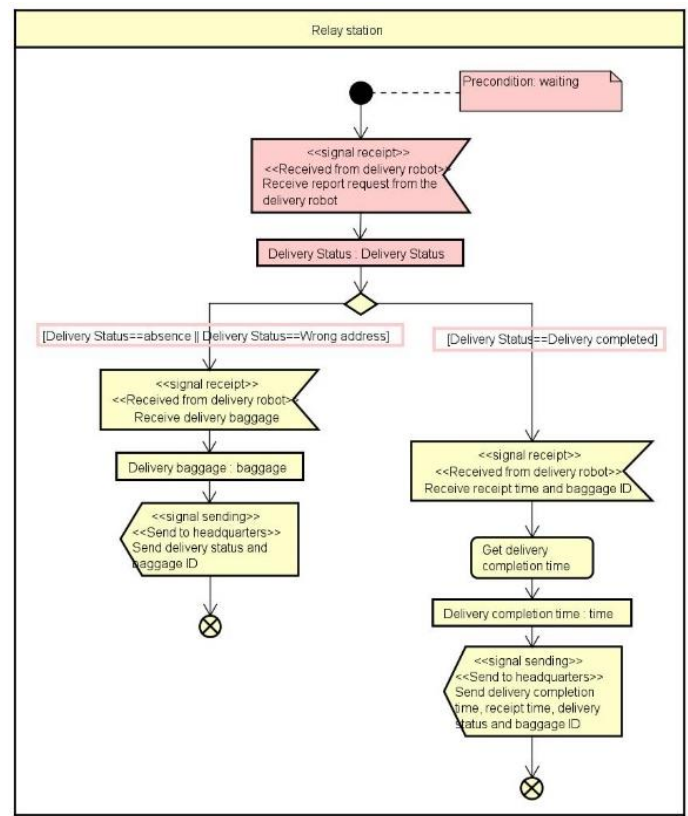

Figure 11: Improved Workflow

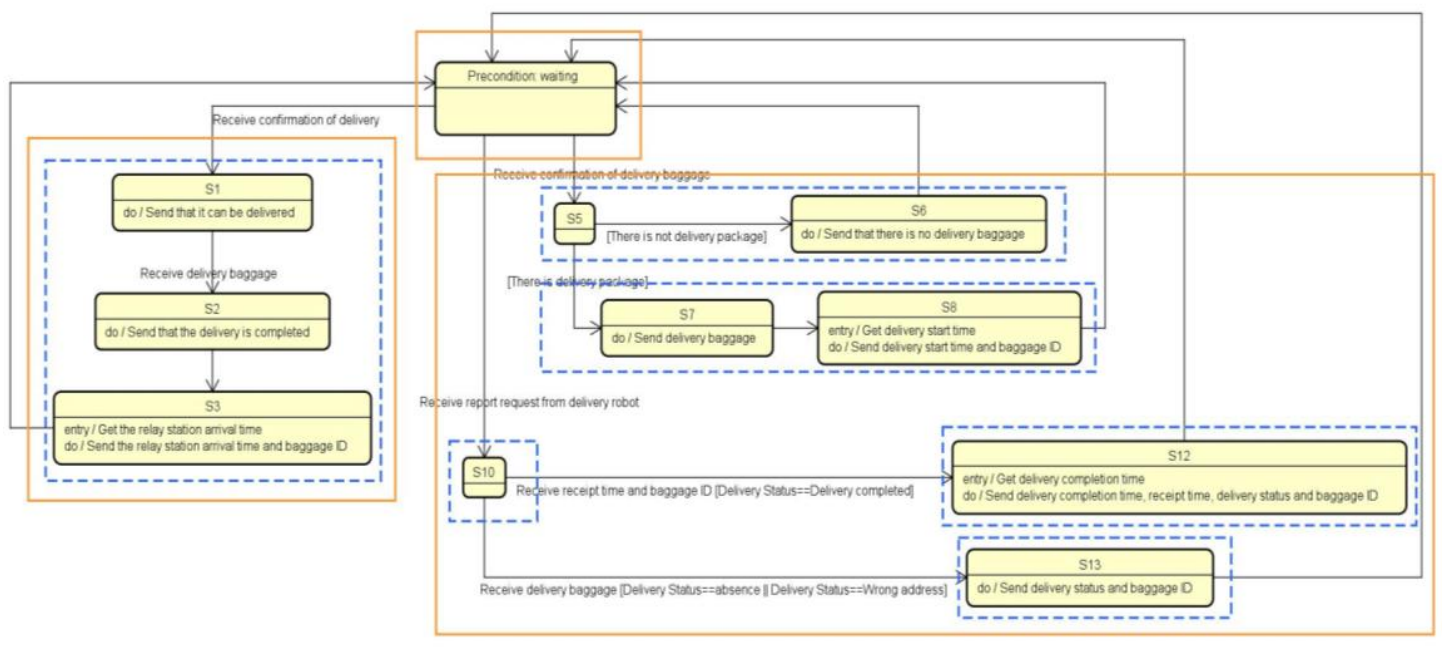

Figure 12: Regenerated Extraction Model

Next, we consider the transitions marked in red with states S10 and S12 as transition sources. The workflow defines the behavior of delivery completion, but the evaluation model does not include a behavior equivalent to delivery completion. As the initial requirements include the behavior at the time of delivery completion, it is clear that the evaluation model is insufficient. Figure 13 
shows the modified evaluation model. The part surrounded by the red dotted line in Figure 13 denotes the added state and state transition.

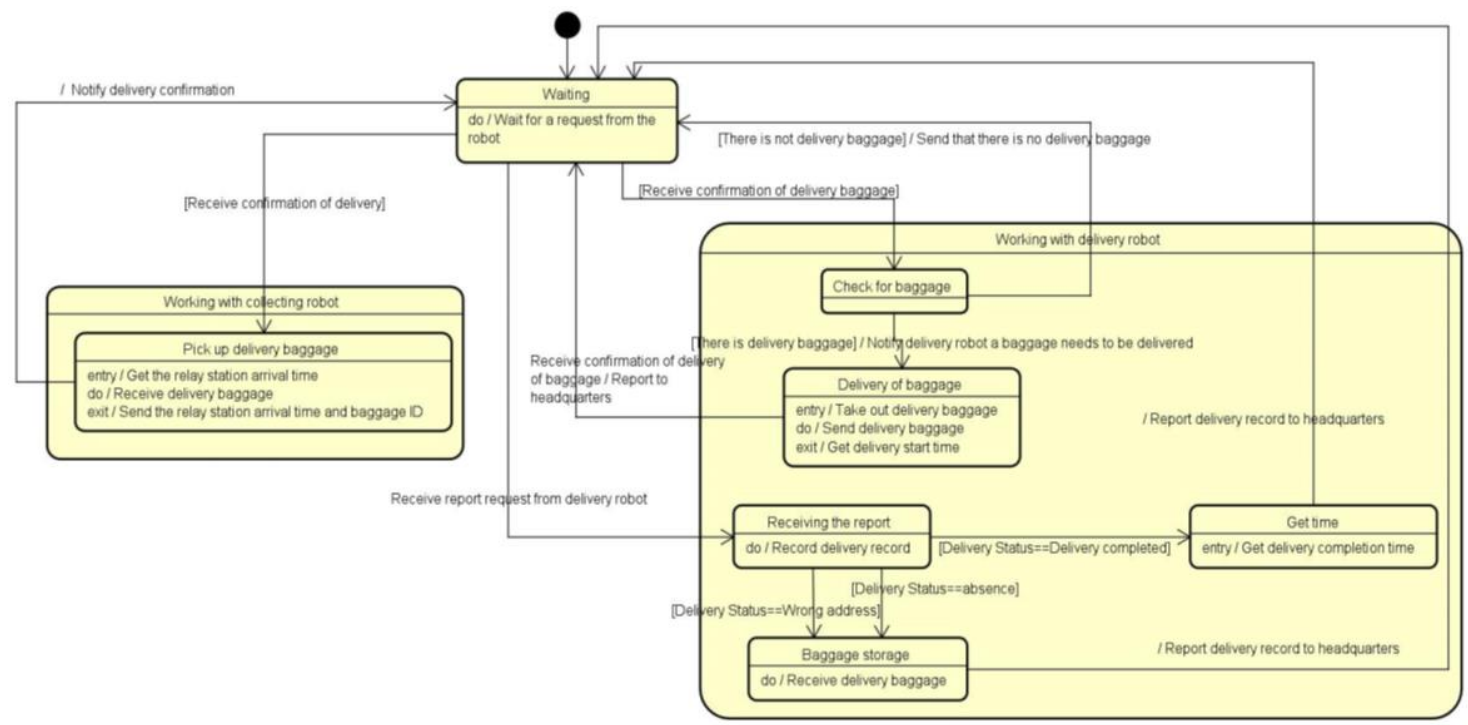

Figure 13: Improved Evaluation Model

After the first layer was modified, the extraction model was regenerated. It was observed that the number of states and transitions in the first layer was equivalent in both models.

Subsequently, the same classification was performed in the second layer. The results of steps 0,1 , and 2 are shown in Table 5, and the results of step 3 are shown in Table 6. Similarly, the number of states and transitions matched in the second layer.

Table 5: Classification by Steps 0,1 , and 2

\begin{tabular}{|l|l|l|l|l|l|}
\hline Check for baggage & Dlivery of baggage & Receiving the report & Baggage Storage & Get time & unknown \\
\hline S6 & S7 & S10 & & & S12 \\
\hline S5 & S8 & & & & \\
\hline
\end{tabular}

Table 6: Result of Step 3

\begin{tabular}{|l|l|l|l|l|l|}
\hline Check for baggage & Dlivery of baggage & Receiving the report & Baggage Storage & Get time & unknown \\
\hline S6 & S7 & S10 & S11 & S12 & \\
S5 & S8 & & & & \\
\hline
\end{tabular}

As the workflow and evaluation model were improved by the difference for all layers of the evaluation model, the improved extraction and evaluation models became a state transition model, in which the states and transitions were equal. Finally, we compared the words of the event, guard, and action, which were the behavior of the transition or the state and determined the excess or deficiency of each behavior.

In reference to the "working with collecting robot" state shown in Figures 11 and 12, the four behaviors can be regarded as having the same content, but the behaviors circled in red showed different expressions. As the expression in the evaluation model was considered to be better, the expression of the action in the workflow was modified. Furthermore, it was discovered that the behavior order was different. The order in the evaluation model appeared to be more appropriate; therefore, the order of actions in the workflow was altered. 


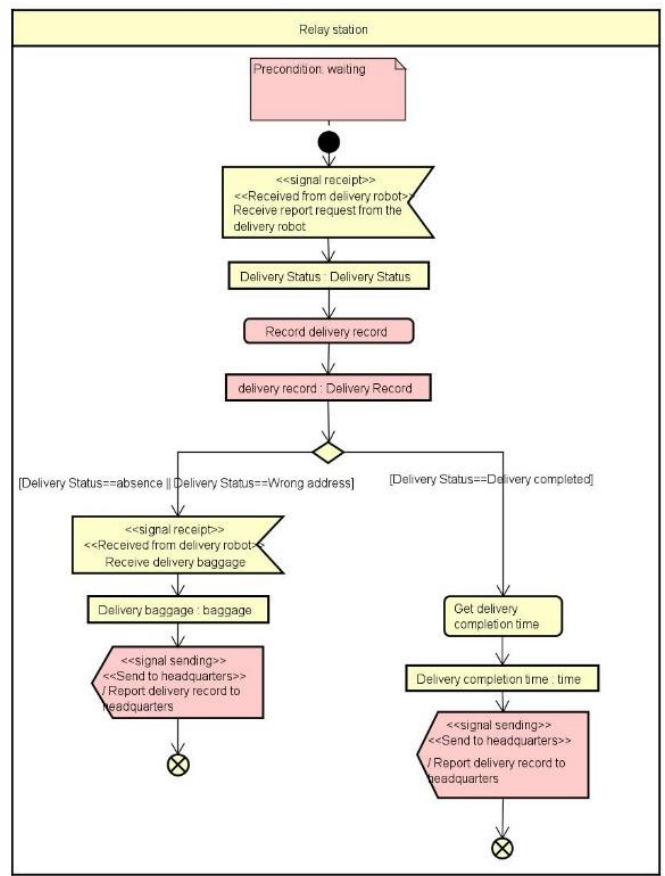

Figure 14: Modified Workflow

Next, we focus on the "working with delivery robot" state shown in Figures 11 and 12. The extraction model did not exhibit the behavior corresponding to the behavior circled in blue in the evaluation model. Because the behavior was insufficient, an action at the part corresponding to the relevant state of the workflow was added. Moreover, in the extraction model of Figure 12, the blue underlined behavior aims to update the "delivery record" such that the representation is modified more clearly. Figure 14 shows a workflow that reflects these modifications. The actions shown in pink in Figure 14 represent the added and modified actions.

\section{CONClusions}

To realize model-driven development in which behaviors are assigned to classes from the requirements analysis models and the final programs are generated from the designed class diagrams, the requirements analysis model must be of high quality. We proposed a validation method to improve the quality of requirements analysis models and developed a support tool.

The support tool was implemented as a plug-in in astah * Professional [7], which is a UML modeling tool. It offers the following two functions:

- A function to generate an extraction model from the workflow of the selected subsystem.

- A function to compare the extraction and evaluation models and present the information regarding the difference.

According to this information, the developer improves the behavior described in the workflow. However, a deficiency might be discovered in the interpretation of requirements in the evaluation model.

In the case study, we discovered differences in interpretation as well as the lack of descriptions such as guard conditions or some states that must be specified in the requirements analysis model. Because it is unclear whether the description of the evaluation model is always 
appropriate, it is possible that truly necessary and unnecessary requirements should be determined by comparing the two different view models and discuss the differences. We plan to apply this approach to various cases and verify its effectiveness.

\section{REFERENCES}

[1] Ogata, S \& Matsuura, S, 2008, A UML-Based Requirements Analysis with Automatic Prototype System Generation, Communication of SIWN, 3, pp. 166-172.

[2] Ogata, S \& Matsuura, S, 2010, A Method of Automatic Integration Test Case Generation from UMLBased Scenario WSEAS Transactions on Information Science and Applications, Vol. 7, No. 4, pp. 598-607.

[3] Saeko Matsuura, Sae Ikeda, Kasumi Yokota, 2020, Automatic Verification of Behavior of UML Requirements Specifications Using Model Checking, MODELSWARD2020, pp. 158-166.

[4] Kaito Yoshino, Saeko Matsuura, 2020, Requirements Traceability Management Support Tool for UML Models, ICSCA2020, A31.

[5] Omar Tariq, Jun Sang, Kanza Gulzar, Hong Xiang, 2017, Automated Analysis of UML Activity Diagram Using CPNs, 2017 8th IEEE International Conference on Software Engineering and Service Science (ICSESS), pp. 134-138.

[6] Vahid Rafe, Reza Rafeh, Somayeh Azizi, Mohamad Reza Zand Miralvand, 2009, Verification and Validation of Activity Diagrams Using Graph Transformation, International Conference on Computer Technology and Development, pp. 201-205.

[7] Astah, http://astah.net/.

\section{AUTHORS}

Hikaru Morita In 2020, enrolled in the master's program at the Department of Systems Science and Engineering, Graduate School of Science and Engineering, the same university. Currently engaged in research in the field of software engineering.

Saeko Matsuura Shibaura Institute of Technology system Professor, Faculty of Science and Technology of Electronics and Information Systems Department in April 2013. Engaged in research on software development environments, design methodologies, and object-oriented development technologies.
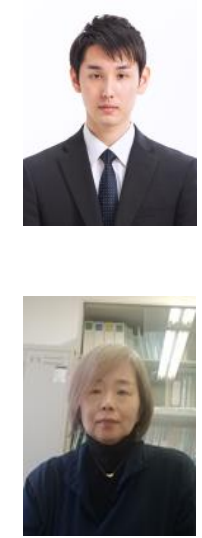

(C) 2021 By AIRCC Publishing Corporation. This article is published under the Creative Commons Attribution (CC BY) license. 


\title{
INTELLIGENT SYSTEM FOR SOLVING Problems of VeTERINARY MEDicine ON THE EXAMPLE OF DAIRY FARMS
}

\author{
Shopagulov Olzhas, Tretyakov Igor and Ismailova Aisulu \\ Dept. of Information Systems, Kazakh Agro Technical University named \\ after S. Seifullin, Nur-Sultan, Kazakhstan
}

\begin{abstract}
This article describes an automated expert system developed to diagnose cow diseases and assist veterinarians in treatment. We set before a diagnostic method based on the analysis of observed symptoms and experience of veterinarians. The system represents a web interface for maintaining a database of diseases, their symptoms and treatment methods, as well as a smartphone application for the diagnostics in offline mode. The developed intelligent system will allow agricultural producers to make specific decisions based on automated data analysis. Also presented in the article the information on the developed expert system, and the results of tests and testing during its use. The economic efficiency and importance of the work is determined by the possibility of automated recording of data on the livestock of animals, zoo technical and veterinary operations.
\end{abstract}

\section{KEYWORDS}

Intelligent system, diagnosis of diseases, application evaluation, milk yield, herd management

\section{INTRODUCTION}

Modern animal husbandry is a transition to new production approaches. The basis of this transformation is agricultural machinery and equipment, supplemented by telemetry systems. Telemetry and monitoring systems for agricultural production are considered one of the most innovative technologies for precision animal husbandry and are automatic systems for collecting and transmitting information, as well as data analysis and remote decision making.

Nowadays, the use of digital technologies in intensive dairy farming is playing a key role in the proper management of the herd to improve animal welfare and increase the milk production of cows. Italian scientists E. Tullo, I. Fontana, D. Gottardo, K. H. Sloth, M. Guarino [1] conducted research on the application of the GEA CowView system (Gea Farm Technologies, Benen, Germany) on dairy cows.

Similar studies were carried out by Turkish scientists on the use of the SCADA application in dairy farming, which allows you to evaluate all records of animal production in a digital environment (behavior, production, health, feeding, and other events). With the introduction of automation systems into industrial production, it creates new work areas in the livestock sector for various applications of emerging technologies [2].

A huge role in the productivity of cattle is played by various diseases. These factors are a significant obstacle to the healthy and sustainable development of livestock breeding. Cattle, in 
particular cows, have a number of specific features in the diagnostics and establishing diagnosis. Taking into account the world experience in developing intelligent systems, the conclusion on their creation has been made. The uniqueness of this system is determined by the development methods used and the knowledge base of veterinary experts. The study objective was to develop an automated tool for diagnostics of cattle diseases and, and study its impact on the diagnostics of cattle diseases in Kazakhstan [3].

Contrary to humans, animals are unable to describe their feelings, and diagnosis of diseases is based on externally observed symptoms and laboratory tests. For example, if a lacrimation is detected in a cow, it may indicate conjunctivitis. But practice shows that in most cases there are other factors that affect the development of disease. Animals do not exhibit obvious clinical symptoms when they suffer from a disease, the former are usually misdiagnosed as a result of the disease. In spite of the improved diagnostics of cow diseases in recent years, most veterinarians do not have much experience, which leads to serious losses due to delayed disease control, as well as serious problems in accounting diseases on paper. Health of cows is a key factor in dairy herd productivity. Mastitis, ketoses, fattening problems and other diseases significantly reduce dairy production and treatment will be expensive if diagnosed and detected late. Thus, the financial component and disease prevention are interlinked phenomena on any farm. Improving diagnostic accuracy and reducing losses caused by disease are the most serious problems at present (3).

Input data needed to diagnose cattle diseases should be classified, i.e. we need to know what information a farmer should have in order to make a decision [3]. Several groups of input data can be identified:

1. External characteristics:

- Sex, breed, age (date of birth), live weight of the animal;

- Genealogical tree of the animal (breeding record);

- Animal data: individual number, body type, live weight, age, color, sex, photo of the animal,

- Place of birth, date of birth and location of the animal;

- Method of birth of the animal (natural / artificial breeding);

- Date of slaughter (and disposal actions following the slaughter).

2. Animal productivity:

- Volume of dairy products produced and milking schedule;

- Live weight at present and graphs of live weight changes;

- Date and method of last animal cover (natural or artificial breeding), date of pregnancy test and its result, date of start in the dry;

- Date of last calving and number of calves (live, stillborn);

3. Animal's medical information:

- Full medical history of the animal (dates of diagnosis, treatment);

- Immunization and vaccination of the animal with the dates and type of medication injected;

- Graph of body temperature changes, mobility, recent PH in the stomach.

4. Observed symptoms and organ and body system lesions: skin cover, musculoskeletal system, nervous system, cardiovascular system and others.

By processing the received input and output data, we should obtain a set of expert conclusions (output data) accepted by the system:

1. A diagnosis of the animal's disease obtained by analyzing the input data and comparing it with the symptom data library;

2. Recommendations for further actions on treatment, prevention, use of medications, etc.; 


\section{General System Design CONCEPT}

The expert system architecture was developed according to the method of structured systems development. It consists of a knowledge base, a knowledge subsystem, a decision-making subsystem, an administration subsystem and a user interface (Figure 1) [3].

The system uses N-tier web architecture (Figure 1), the structure has been developed according to the method of development of structured systems, consisting of:

1. Knowledge databases and knowledge generation subsystems (interface for working with veterinary experts);

2. System administration block and the administrator work interface correspondingly;

3. Database and data management block are the core of the system;

4. Work with input and output data as well as decision making and work with the user interface is located in the disease diagnostic subsystem.

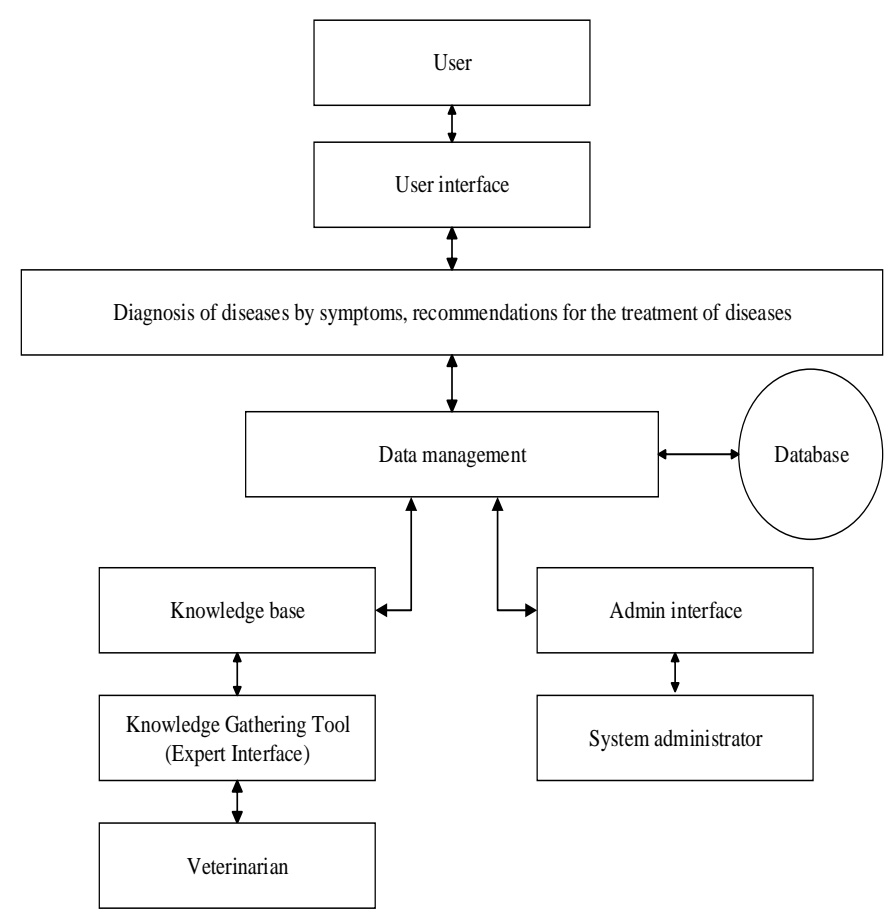

Figure 1: Structure of a cow disease diagnosis system

Decision making diagram of the system is used for interpretation of user interface functions. Diagram of variants of use of expert system of diagnostics of diseases of the cows consists of six compound blocks shown in Fig. 2. 


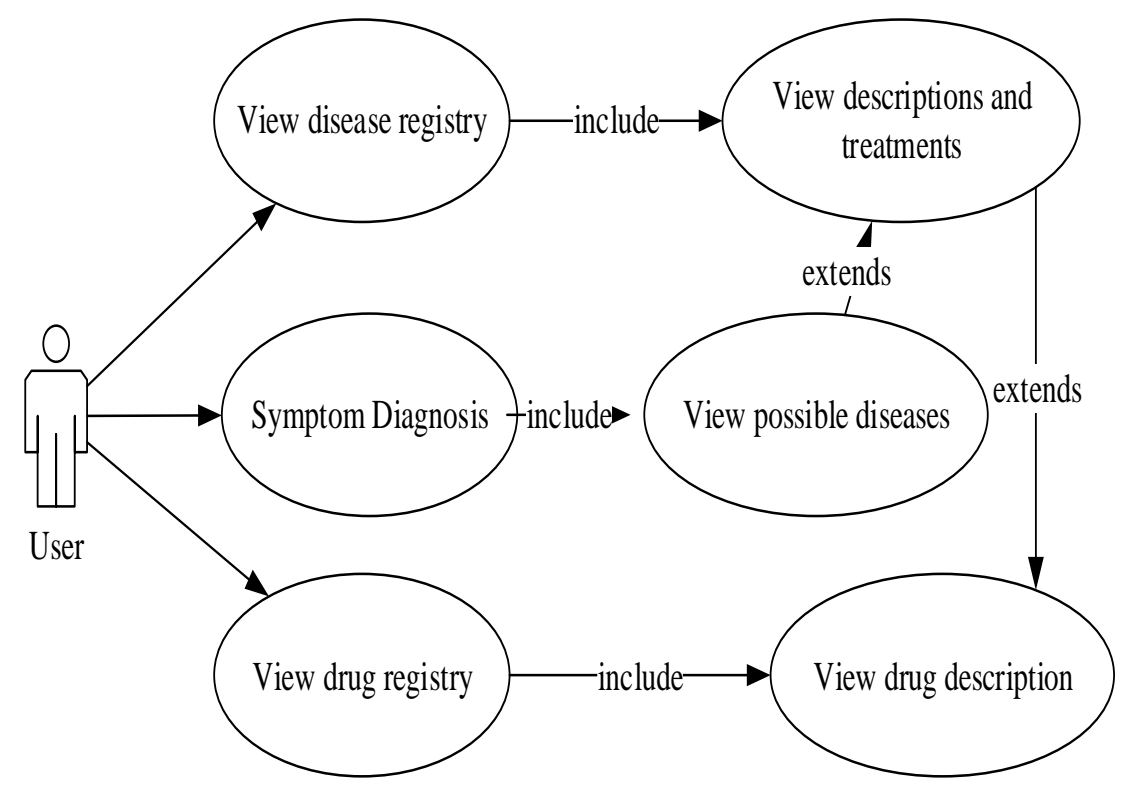

Figure 2: The main scenarios for using the expert system

Figure two shows that a user entity in the system can perform a number of actions, such as viewing the disease registry, performing diagnostics of diseases by symptoms and viewing the medication registry. On this basis, having defined a diagnosis by initial symptoms, the system will offer a description, methods of treatment and prevention of this disease. After selecting a treatment method, it is possible to view the descriptions of the medications used [3].

\section{ACQuisition And Presentation of KnOWledge}

As expert system, it should contain knowledge obtained from experts in the subject area. Acquisition and presentation of knowledge is the most important stage in building expert systems. The main task is to create a knowledge database to meet the requirements of the expert system for the solution of set tasks. Many methods were developed for obtaining knowledge from experts in the subject area.

In this study, we have analyzed and summarized the aggregate of knowledge by conducting literature reviews and interviewing experts using a questionnaire for disease analysis. It consisted of questions about symptoms, diagnoses and treatments. Based on their experience and requirements to develop an expert system, experts modified the questionnaire and provided information in tabular form [8].

In this study the diagnosis of 16 most dangerous infectious diseases of cattle is considered: Anthrax (D1), FMD (Foot-and-Mouth Disease) (D2), Tuberculosis (D3), Brucellosis (D4), Rabies (D5), Pasteurellosis (D6), Trichophytosis (D7), Leukosis (D8), Infectious Rhinotracheitis (D9), Viral Diarrhea (D10), Lumpy Skin Disease of Cattle (D11), Emphysematous Carbuncle in Cattle (D12), Salmonellosis (D13), Colibacillosis (D14), Rotavirus (D15), Coronavirus (D16). 


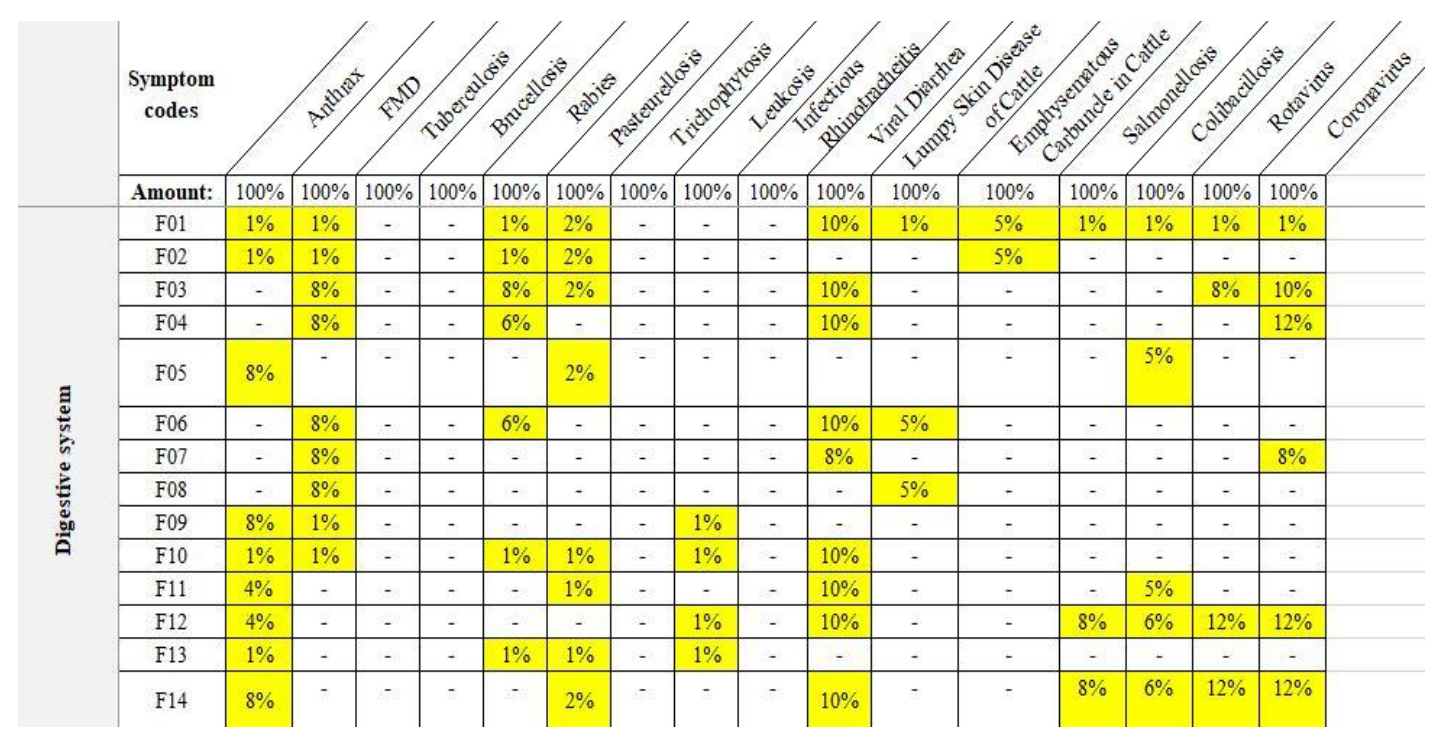

Figure 3: Table of symptoms and their weighting values

Symptoms of disease are grouped according to the affected organs and systems, and each symptom is assigned a code for ease of work:

Skin: Skin Lesions (S01), Rumpleness of Hair (S02), Dermatitis (S03), Lumpy Skin, Extuberances (S04), Papules (S05), Vesicles (S06), Pustules (S07), Sloughs (S08), Scaliness (S09), Scratch (S10), Dark Red Skin Stains (S11).

Musculoskeletal system: Lesion of Limbs (M01), Arthritis (M02), Bursitis (M03), Limp (M04), Bone Deformity (M05), Suppurative Discharge (M06), Edema of Limbs (M07), Edema in Groats, Lungs, Neck, Chest, Lower Jaw (M08), Edema of Joints (M09), Joint Deformity (M10).

Digestive system: Loss of Appetite (F01), Loss of Chewing Cud (F02), Salivation (F03), Stomatitis (F04), Oral Mucosa Hemorrhages (F05), Oral Lesions (F06), Aphta, Oral Ulcers (F07), Vesicles, Tubercules in Mouth (F08), Gastric Timpany (F09), Gastric Atony (F10), Abdomen Wall Disease (F11), Diarrhea (F12), Constipation (F13), Excrements admixed with blood, mucus, gas bubbles (F14).

Respiratory system: Respiratory System Injury (B01), Rapid Pulse (B02), Short Wind (B03), Nasal Cavity Excretion (B04), Injury of Nasal Cavity and Tapetum Lucidum Cellulosum (hemorrhages, wounds, etc.), (B05), Coughing (B06), Lung Rale (B07), Lung Inflammation (B08), Edema in Larynx, Chin, Abdomen (B09).

Central nervous system (CNS): Injury of the CNS (N01), Excitation (N02), Inhibition (N03), Muscular Tremor (N04), Eclampsia (N05), Paresis (N06), Paralysis (N07), Ataxia (N08), Scratch, Pruritus (N09).

Cardiovascular system (CVS): CVS Lesion (H01), Asphyxiation of Mucous Membranes (H02), Mucous Membrane Anemia (H03), Mucous Membrane Hemorrhage (H04), Mucous Membrane Hyperaemia (H05), Tachycardia (H06), Arrhythmia (H07), Myocarditis (H08).

Urogenital system (G-U System): Lesions of G-U System (U01), Nebulous Urine (U02), Urine Erythrocytes (U03), Frequent and Painful Urination (U04), Abortions (U05), Orchids and Epididymitis (U06), Swelling of External Genitals (U07), Hyperemia of External Genitals (U08), 
Viral Shedding of External Genitals (U09), Retention of Placenta (U10), Endometritis (U11), Lesion of Ovaries and Fallopian Tubes (U12), Vulvovaginitis (U13), Balanopostitis (U14).

Visual organs: Lesions of Visual Organs (E01), Mucous membrane bleeding (E02), Eyeball Retraction (E03), Photophobia (E04), Serous or Suppurative Discharge (E05), Conjunctival Hyperemia (E06), Swelling and Edema of Conjunctival (E07), Corneal Ulceration and Clouding (E08).

Lacteous Gland: Lacteous Gland Lesion (J01), Decreased or Stopped Secretion (J02), Afta, Dug Skin Erosions (J03), Dug Inflammation (J04), Udder Edema (J05), Udder Pain (J06), Mastitis (J07), Enlarged Supramammary Glands (J08), Watery Milk admixed with Blood or Curds (J09).

Lymphoid system: Lesion of Lymphoid System (L01), Inflammation of Submandibular and retropharyngeal Lymphnodes (L02), Inflammation of Prescapular Lymph Glands (L03), Inflammation of Precrural Nodes (L04), Inflammation of Parotid Lymphnodes (L05), Inflammation of Supramural Nodes (L06), Inflammation of Internal Lymphnodes (L07), Tumour Proliferation (L08), Enlarged Spleen (L09).

Based on their own experience, the experts attached a weighting factor (w) to each symptom. The concept of "symptom complex" has also been introduced to reflect a group of symptoms combined into a single value argument, which is also given a weighting value (w). Figure 3 shows some symptom values and weighting values [3].

In order to determine the most probable diseases, the system calculates the sums of the weighting values for the observed symptom complexes and individual symptoms, after which the calculated values and the corresponding diseases are ranked (sorted) in descending order.

\section{Method of Calculation}

According to the method of weighting calculation, we can analyze a given case on a concrete example. Viral diarrhea disease, with a certain number of symptoms and symptom complexes. According to the knowledge database and the questionnaire received from veterinary experts, the following symptoms appear in case of viral diarrhea:

$\mathrm{C} 01$ (Fever) $\mathrm{W}(\mathrm{d}, \mathrm{s})=1 \%$;

M01 (Lesion of Limbs) W(d, s)=1\%;

M04 (Limp) W(d, s)=1\%;

F01 (Loss of Appetite) W(d, s)=10\%;

F03 (Salivation) W(d, s) $=10 \%$;

F04 (Stomatitis) W(d, s) $=10 \%$;

F06 (Oral Lesions) W(d, s) $=10 \%$;

F07 (Aphta, Oral Ulcers) W(d, s)=8\%;

F10 (Gastric Atony) W(d, s)=10\%;

F11 (Abdomen Wall Disease) W(d, s) $=10 \%$;

F14 (Excrements admixed with blood, mucus, gas bubbles) $\mathrm{W}(\mathrm{d}, \mathrm{s})=10 \%$;

N01 (Injury of the CNS) W(d, s)=6\%;

N03 (Inhibition) $\mathrm{W}(\mathrm{d}, \mathrm{s})=1 \%$;

N08 (Ataxia) W(d, s) $=1 \%$,

where C01 is the symptom code, W (d, s) is the weight value of symptom s for disease $\mathrm{d}$. 
Thus, it is possible to calculate the sum of weighting coefficients of symptoms by disease. A simple sum of weights of symptoms for the disease is calculated using a formula:

$$
W\left(d, S^{0}\right)=\Sigma_{s \in S^{\infty}} W(d, s)
$$

where $\mathrm{d}$ is the disease, So is the observed set of symptoms, W (d, s) is the weighting value of symptom $\mathrm{s}$ for disease $\mathrm{d}$.

For the above example with the disease "viral diarrhea», the values of the arguments will be equal to:

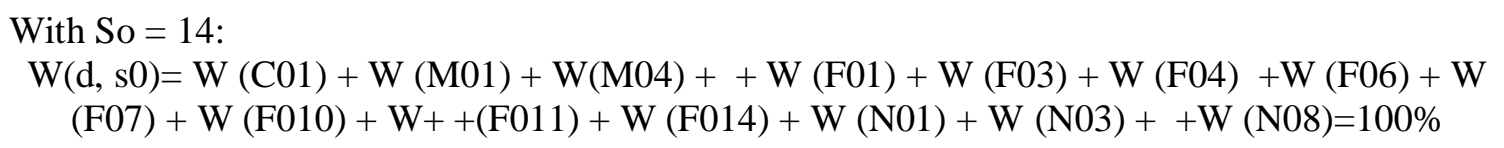

Due to the fact that several symptom complexes $\mathrm{k}$ with different weights $\mathrm{w}$ can be defined for disease $\mathrm{d}$, the symptom complex with the highest weighting is taken into account, each symptom of which includes the many symptoms observed:

$$
\begin{aligned}
& k_{\max } \in K(d) \text {, где } S(k) \in S^{\circ}{ }_{s} \text { и } W(d, k) x \\
& \text { i.e. } W(d, k) \geq W\left(d, k_{j}\right) \text { for } \forall k_{j} \in K(d)
\end{aligned}
$$

where: $\mathrm{K}(\mathrm{d})$ - symptom complexes of the disease $\mathrm{d}, \mathrm{S}(\mathrm{k})$ is the set of symptoms of symptom complex $\mathrm{k}, \mathrm{W}(\mathrm{d}, \mathrm{k})$ - weight coefficient of symptom complex $\mathrm{k}$, for disease $\mathrm{d}$.

Thus, for viral diarrhea, the symptom complex with the highest weighting factor is $\mathrm{k}_{\max }$, which includes symptoms F01, F03, F04, F06, F07, i.e. $\mathrm{S}\left(\mathrm{k}_{\max }\right)=5$.

In total for the disease "viral diarrhoea" the symptom complex $\mathrm{k}_{\max }$ will give $\mathrm{W}\left(\mathrm{d}, \mathrm{k}_{\max }\right)=65 \%$, according to the knowledge base provided by veterinary experts.

Taking into account the above mentioned number of symptoms not included in the symptom complex will be calculated as $\mathrm{Sx}=\mathrm{S} 0-\mathrm{S}\left(\mathrm{k}_{\max }\right)$ difference, i.e. for viral diarrhea $\mathrm{Sx}=9$. Thus, it is easy to calculate $\mathrm{W}(\mathrm{d}, \mathrm{Sx})=52 \%$.

The total sum of weights $\mathrm{R}$ for observed symptoms So and symptom complexes $\mathrm{S}\left(\mathrm{k}_{\max }\right)$ for disease $\mathrm{d}$ is calculated by formula [12] :

$$
W_{r}(d, S)=W\left(d, k_{\max }\right)+W\left(d, S^{y}\right)
$$

For viral diarrhoea, the total sum of $\mathrm{R}$ weights for the observed symptom group Sy (F11, F14) and symptom complexes $\mathrm{S}\left(\mathrm{k}_{\max }\right)$ at the same time will be $\mathrm{Wr}(\mathrm{d}, \mathrm{S})=85 \%$.

After calculating the total sums, the obtained data are sorted in descending order.

Thus, we can conclude that the introduction of such a parameter as a symptom complex leads to a more accurate definition of this or that disease. The results obtained as a percentage may show that the observed symptoms belong to a certain diagnosis [3]. 


\section{DATABASE, THEIR IMPLEMENTATION}

For the central database as well as for its local version, relational databases (MS SQL Server 2019 and SQLite) are used on the user device. Figure 4 presents the database structure in terms of knowledge storage about diseases, symptoms and symptom complexes [3].

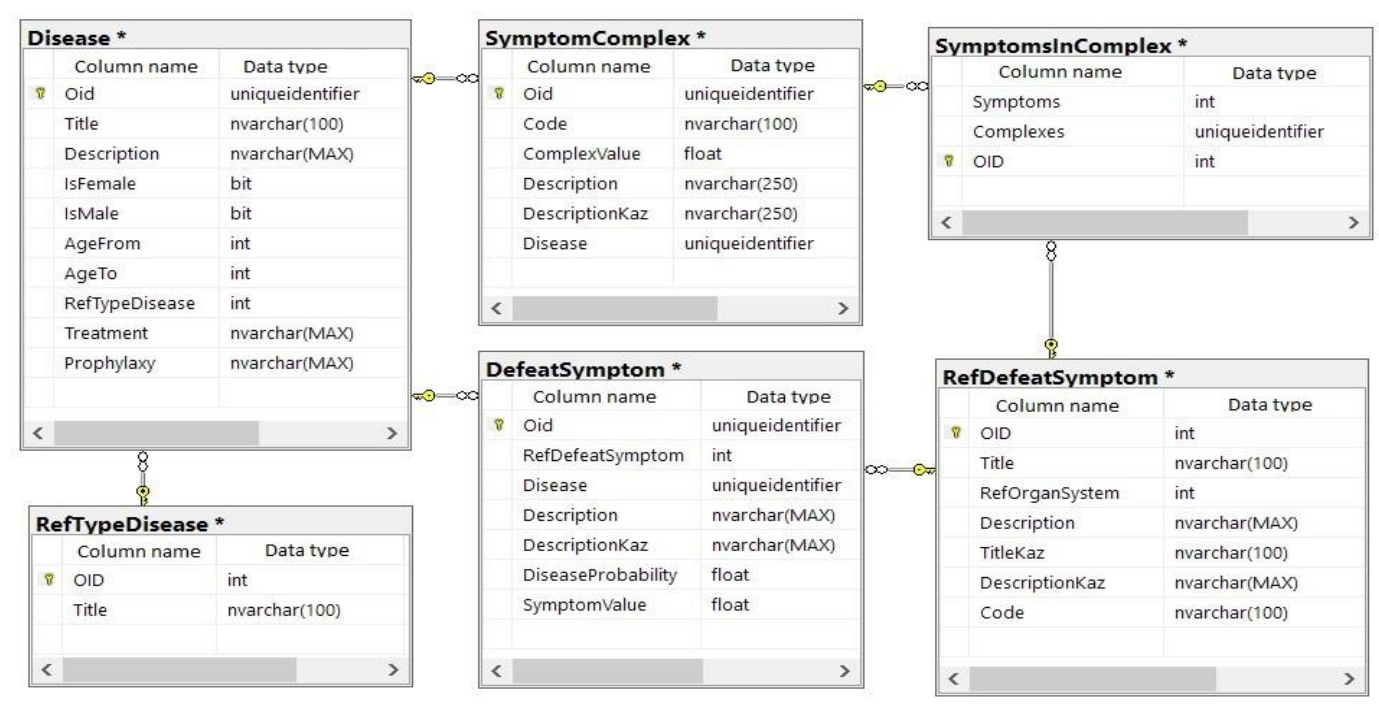

Figure 4: Database structure

Knowledge database contains information about 16 major infectious diseases and 103 symptoms of diseases. The database stores the subject area knowledge needed to solve problems, including age, cow breed, symptoms, photographs, and other relevant information. The database is developed on:

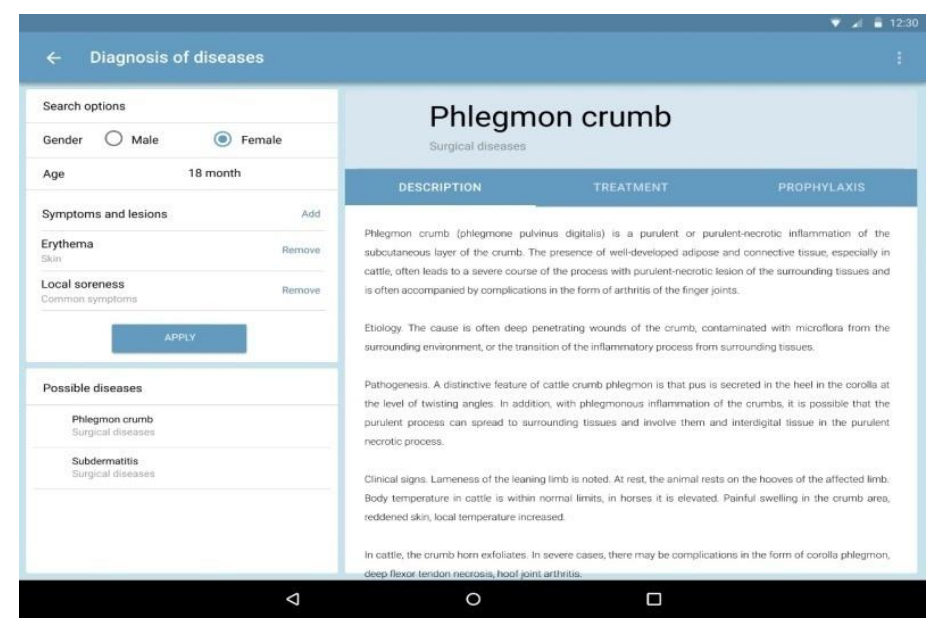

Figure 5: Veterinarian tablet web interface

- Operating system: Windows Server 2019 Standard;

- Web server: Internet Information Services;

- DBMS: SQL Server 2017 Standard;

- Platform: NET 4.5.2, language C \#; 
- Framework: DevExpress XAF 18.2 - a set of libraries to help the developed program with modern high-quality functionality [3].

\section{EXPERT System Evaluation - TeSt ANd Results}

The evaluation process was carried out due to the user-friendliness of the user interface and system utilization efficiency testing. The reliability of the system diagnosis was evaluated with the participation of two groups of senior veterinary students of KATU named after S. Seifullin.

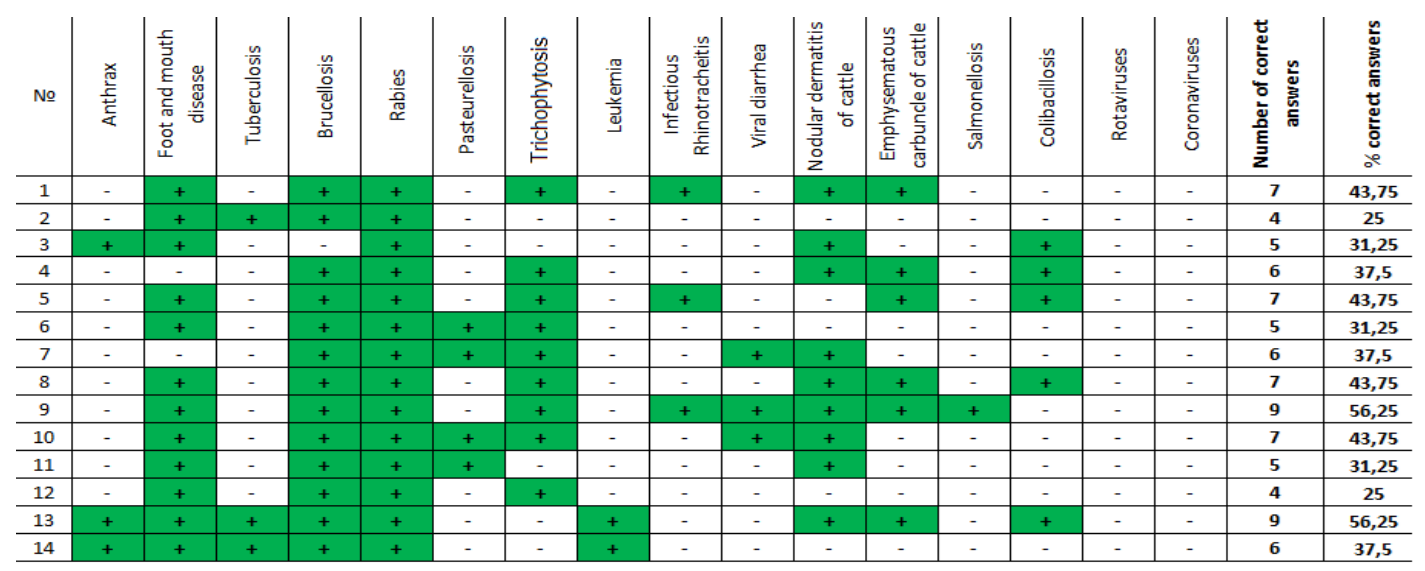

Figure 6: Questionnaire of Veterinary Students

Table 1: Changes Characteristic in the Disease Diagnosis Using Tablet versus Diagnosis without Tablet

\begin{tabular}{|c|c|c|}
\hline $\begin{array}{l}\text { Job } \\
\text { Number }\end{array}$ & $\begin{array}{l}\text { Name of the } \\
\text { disease }\end{array}$ & $\begin{array}{l}\text { Characterization of changes in the diagnosis of the disease when using a } \\
\text { tablet, compared with diagnosis without a tablet }\end{array}$ \\
\hline 001 & Anthrax & $\begin{array}{l}\text { A significant increase in the probability of diagnosing anthrax - from } 20 \% \\
\text { to } 69 \% \text { ( } 3.43 \text { times) }\end{array}$ \\
\hline 002 & $\begin{array}{l}\text { Foot and } \\
\text { mouth disease }\end{array}$ & $\begin{array}{l}\text { The increase in the probability of diagnosis of foot and mouth disease - from } \\
70 \% \text { to } 93 \% \text { ( } 1.33 \text { times). }\end{array}$ \\
\hline 003 & Tuberculosis & $\begin{array}{l}\text { A significant increase in the probability of diagnosis of tuberculosis - from } \\
12 \% \text { to } 89 \% \text { (7.41 times) }\end{array}$ \\
\hline 004 & Brucellosis & $\begin{array}{l}\text { slight decrease in the probability of diagnosis of brucellosis - from } 82 \% \text { to } \\
1 \%\end{array}$ \\
\hline 005 & Rabies & $\begin{array}{l}\text { slight decrease in the probability of a correct diagnosis of rabies is from } \\
0 \% \% \text { to } 92 \% \text {. Probably by providing subjects with additional options }\end{array}$ \\
\hline 006 & Pasteurellosis & $\begin{array}{l}\text { in the probability of correct diagnosis of pasteurellosis - from } \\
\% \text { (1.23 times) }\end{array}$ \\
\hline 007 & nytosi & A slight increase in the probability of a correct diagnosis from $68 \%$ to $74 \%$ \\
\hline 008 & Leukemia & ct diagnosis of leukemia - \\
\hline 009 & cheiti & $\begin{array}{l}\text { Decrease in probability of correct diagnosis of infectious rhinotracheitis - } \\
\text { from } 54 \% \text { to } 44 \%\end{array}$ \\
\hline 010 & Viral diarrhea & $\begin{array}{l}\text { slight increase in the probability of a correct diagnosis of viral diarrhea - } \\
\text { om } 20 \% \text { to } 22 \%\end{array}$ \\
\hline 011 & $\begin{array}{l}\text { Nodular } \\
\text { dermatitis } \\
\text { cattle }\end{array}$ & $\begin{array}{l}\text { Increasing the likelihood of a correct diagnosis of nodular cattle dermatitis - } \\
\text { from } 80 \% \text { to } 90 \% \text { (1.13 times). }\end{array}$ \\
\hline 012 & $\begin{array}{l}\text { Emphysemato } \\
\text { us cattle } \\
\text { carbuncle }\end{array}$ & $\begin{array}{l}\text { An increase in the probability of a correct diagnosis of cattle } \\
\text { emphysematous carbuncle - from } 58 \% \text { to } 67 \% \text { (1.15 times). }\end{array}$ \\
\hline 013 & Salmonellosis & Reducing the likelihood of a correct diagnosis of salmonellosis - from $40 \%$ \\
\hline
\end{tabular}




\begin{tabular}{|r|l|l|}
\hline & & to 35\%. \\
\hline 014 & Colibacillosis & $\begin{array}{l}\text { An increase in the probability of correct diagnosis of colibacillosis - from } \\
38 \% \text { to 58\% (1.52 times) }\end{array}$ \\
\hline 015 & Rotaviruses & $\begin{array}{l}\text { A significant increase in the probability of correct diagnosis of rotavirus } \\
\text { infections - from 8\% to 25\% (3.13 times). }\end{array}$ \\
\hline 016 & Coronaviruses & $\begin{array}{l}\text { The correct diagnosis when using the tablet was 25\%, without using the } \\
\text { tablet, no one was tested correctly }\end{array}$ \\
\hline
\end{tabular}

In Figure 6: green indicates that this student has answered the question correctly, and at the end of the table is information on the questions correctly answered and their proportion compared to the total number of tasks.

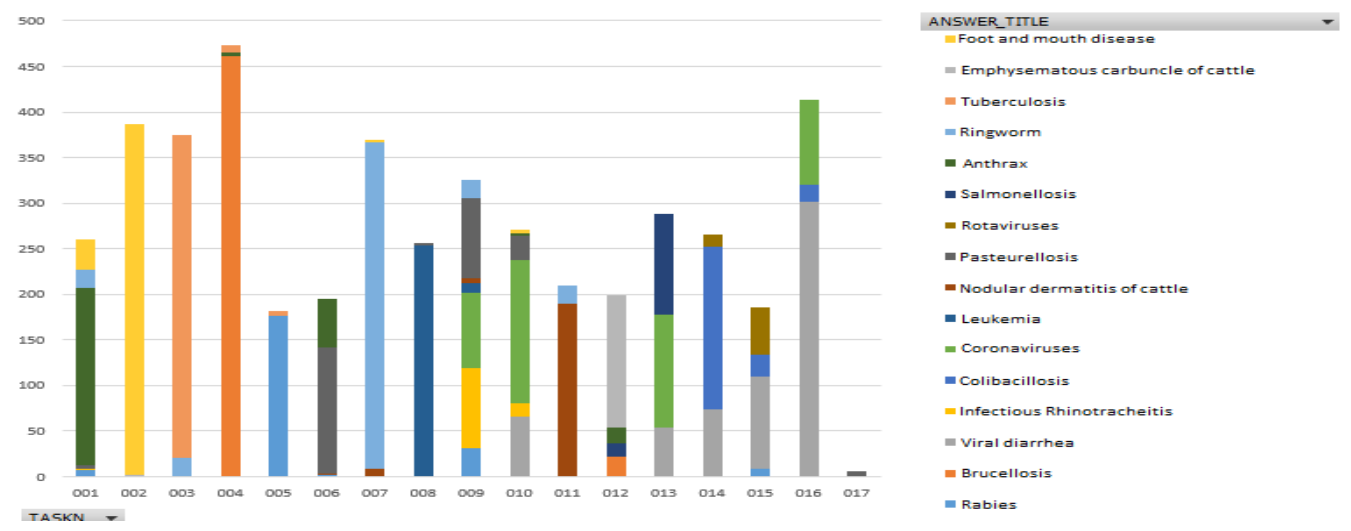

Figure 7: Diagram of Correct Answers Quality Representation

The second group of students worked with the "Veterinary Tablet". This group worked on the same test tasks as the first one. Figure 7 provides information on the quantity and quality of correct answers. After calculating and analysis of the data obtained, we concluded that the percentage of correct answers using the "Veterinary Tablet" was $69 \%$.

As the diagram in Figure 7 shows, most students answered the questions correctly. Colors show the ratio of their answers (diagnosis) depending on the task number. The right column of the diagram shows the colors that correspond to a certain diagnosis of the disease, for example, brucellosis is indicated in orange, and etc.

We have analyzed the most common symptoms that students chose when answering questions using the tablet, analyzed the number of selected symptoms and their types [3].

According to Figure 8, the most frequent symptoms as a result of the test were: fever, stomatitis, salivation, lameness. The rest of the symptoms were less frequent.

After comparing all the data obtained, we came to the conclusion about the probability of making the right diagnosis in cases with and without the application. This information is shown in Table 1.

Table 1 shows the changes characteristic in the disease diagnosis using tablet versus diagnosis without Tablet. The analysis of changes in correct answers taking into account each symptom and set of symptoms is performed.

The average time to answer each question has also been calculated. This information is reflected in Table 2. 
According to Table 2, we can conclude that the average time to conduct diagnostics for known symptoms is $2-5$ minutes. There are no dependencies between the quality of the diagnosis and the time spent on the test task.

For diseases such as leukaemia, tuberculosis, anthrax, rotavirus infections, a clear improvement in the correct diagnosis with help of the veterinary tablet was found (a total of 12 out of 16 test questions showed an improvement in the quality of diseases diagnosis). Separately considering rotavirus infections, the developed software allowed the correct diagnosis of diseases in a quarter of cases, given that without the veterinary tablet no test subjects in this case answered the questions correctly.

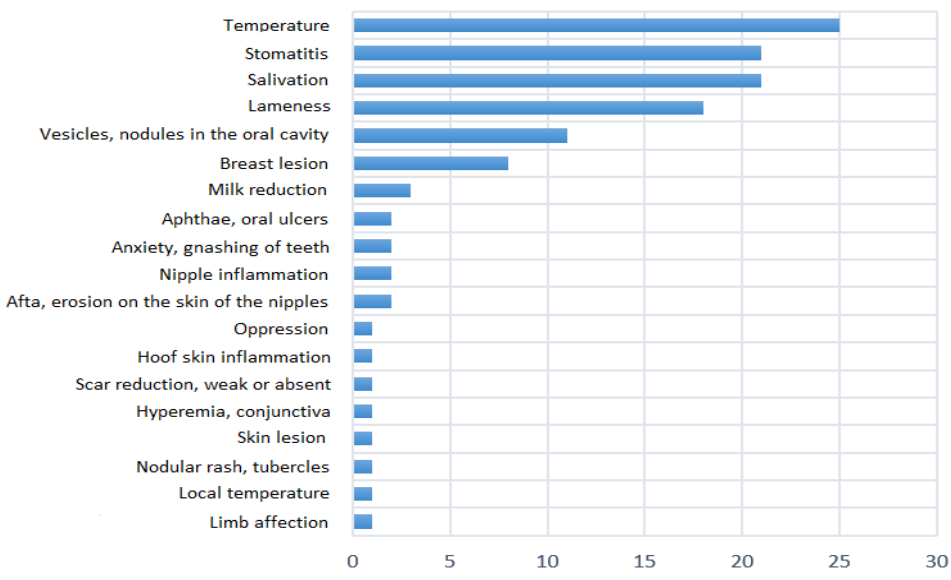

Figure 8: Symptom Distribution Using "Veterinary Tablet"

Table 2: Characteristics of disease diagnosis time with the use of veterinary tablet

\begin{tabular}{cccccccc}
\hline & \multicolumn{2}{c}{ Time to } & make & an & erroneous & \multicolumn{2}{c}{ Time to make the correct diagnosis } \\
diagnosis (seconds) & & & (seconds) & \\
Row & Min. & Avg. & Max. & Min. & Avg. & Max. \\
\hline 001 & 72 & 255 & 938 & 82 & 213 & 375 \\
002 & - & - & - & 62 & 125 & 284 \\
003 & 181 & 181 & 181 & 65 & 113 & 201 \\
004 & 123 & 301 & 478 & 61 & 165 & 540 \\
005 & - & - & - & 64 & 163 & 771 \\
006 & 95 & 185 & 700 & 61 & 142 & 294 \\
007 & 111 & 201 & 338 & 62 & 148 & 539 \\
008 & 89 & 137 & 185 & 64 & 162 & 622 \\
009 & 86 & 246 & 937 & 94 & 255 & 584 \\
010 & 71 & 207 & 456 & 78 & 160 & 346 \\
011 & 83 & 110 & 136 & 60 & 132 & 276 \\
012 & 111 & 157 & 205 & 62 & 244 & 1255 \\
013 & 70 & 227 & 900 & 75 & 112 & 198 \\
014 & 60 & 93 & 146 & 74 & 142 & 313 \\
015 & 63 & 159 & 632 & 64 & 129 & 243 \\
016 & 77 & 123 & 319 & 99 & 131 & 185 \\
Total: & $\mathbf{9 2}$ & $\mathbf{1 8 4}$ & $\mathbf{4 6 8}$ & $\mathbf{7 0}$ & $\mathbf{1 5 8}$ & $\mathbf{4 3 9}$
\end{tabular}

Summing up, we can conclude that the software implemented has improved, on average, the results of correct diagnosis from $42 \%$ to $69 \%$ [3]. 
In the process, some shortcomings of the selected software testing method were revealed. Firstly, it is the impossibility of veterinarians to work directly with the studied animal directly, as well as the limited initial data on disease symptoms and the lack of visibility of the whole picture in general.

\section{Development of an Algorithm for Processing Digital data for Monitoring the Productivity of Farm Animals with ELEMENTS OF MACHINE LEARNING}

One of the main tasks of the work is the development of software for predicting the productivity of farm animals using data from the smaXtec bolus system and data on milk production of animals. The smaXtec system allows real-time reception of such indicators as: $\mathrm{pH}$, body temperature of the animal, measurement of movement activity, etc. The bolus is injected into the rumen of the animal, and then enters the ruminant stomach, the reticulum, and transfers the data from there. An orally administered bolus measures temperature and activity (via an accelerometer), continuously at 10-minute intervals, while the activity measurement is independent of rumen mobility. Typical increases in activity during sexual activity are detected immediately and trigger notifications. The individual activity levels of the cow are taken into account when processing the data. Fever events are presented to the farmer as a graph or list in the cow's profile and in the fertility section of the dashboard. In this way, the history of previously successful inseminations of dairy cows can be documented in the software for calculating the expected lactation. We used the data of the cattle herd LP "Mambetov and K", consisting of 800 heads of the "simmental" population in the period from 2019 to 2020. (for about 30 thousand data points for all animals).

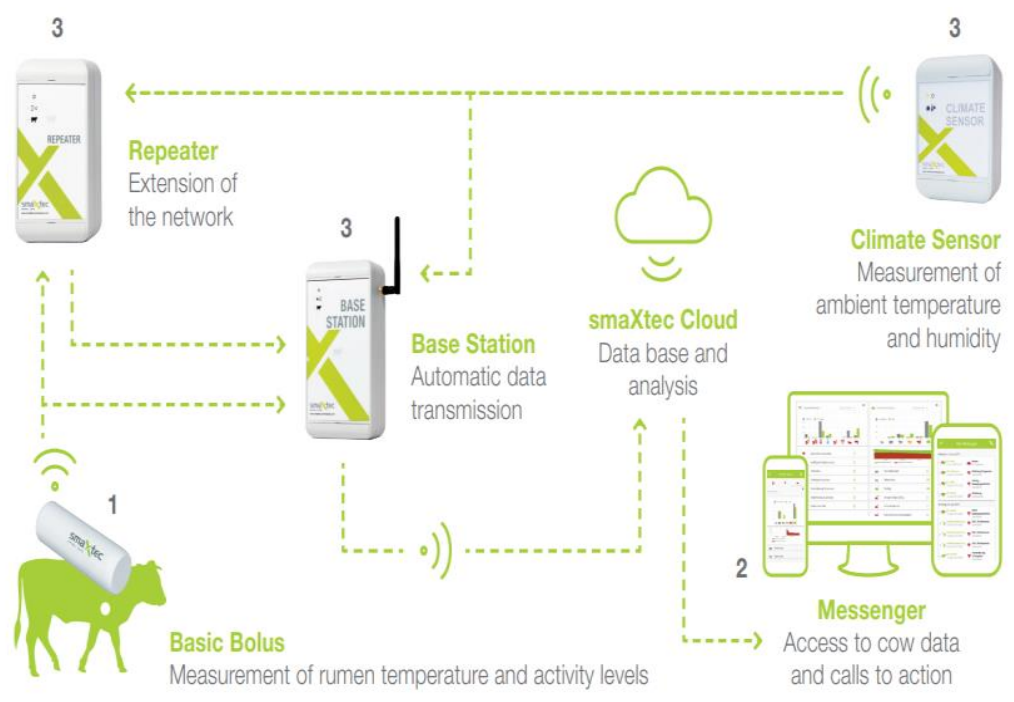

Figure 9: Architecture of the smaXtec system. The smaXtec solution consists of a reticuluminal bolus (1), an online data platform (2), infrastructure including readers and climate sensors (3). 
Table 3: Data for each animal

\begin{tabular}{|c|c|c|c|c|c|c|}
\hline Animal ID & Date & $\begin{array}{l}\text { Body } \\
\text { temperatu } \\
\text { re }\left({ }^{\circ} \mathrm{C}\right)\end{array}$ & $\begin{array}{l}\text { Normal } \\
\text { body } \\
\text { temperatur } \\
\text { e }\left({ }^{\circ} \mathrm{C}\right)\end{array}$ & $\begin{array}{l}\text { Index of } \\
\text { the activity }\end{array}$ & $\begin{array}{l}\text { Body } \\
\text { temperatur } \\
\text { e without } \\
\text { water } \\
\text { consumpti } \\
\text { on }\left({ }^{\circ} \mathrm{C}\right)\end{array}$ & $\begin{array}{l}\text { Milk } \\
\text { yield } \\
(\mathrm{kg}))\end{array}$ \\
\hline DE0667033081 & 18.10.2019 & 39.19 & 40.000000 & 13.223118 & 39.826875 & 6.35 \\
\hline DE0667033081 & 18.10.2019 & 39.19 & 40.000000 & 13.223118 & 39.826875 & 10.34 \\
\hline DE0667033081 & 19.10.2019 & 39.34 & 40.000000 & 14.251181 & 39.871319 & 6.45 \\
\hline DE0667033081 & 19.10 .2019 & 39.34 & 40.000000 & 14.251181 & 39.871319 & 0.00 \\
\hline DE0667033081 & 19.10 .2019 & 39.34 & 40.000000 & 14.251181 & 39.871319 & 10.49 \\
\hline$\cdots$ & $\cdots$ & $\cdots$ & $\cdots$ & $\cdots$ & $\cdots$ & $\cdots$ \\
\hline DE0953378924 & 21.12 .2020 & 38.69 & 40.000000 & 10.522472 & 39.016806 & 0.00 \\
\hline DE0953378924 & 21.12 .2020 & 38.690951 & 40.000000 & 10.522472 & 39.016806 & 4.99 \\
\hline DE0953378924 & 23.12 .2020 & 38.826500 & 40.000000 & 14.486500 & 39.186528 & 5.14 \\
\hline DE0953378924 & 23.12 .2020 & 38.826500 & 40.000000 & 14.486500 & 39.186528 & 0.00 \\
\hline DE0953378924 & 23.12 .2020 & 38.826500 & 40.000000 & 14.486500 & 39.186528 & 4.77 \\
\hline
\end{tabular}

In accordance with the tasks set, such indicators were selected:

1. Body temperature of the animal;

2. Normal body temperature of the animal;

3. Activity index;

4. Body temperature without drinking cycle;

5. Milk yield;

Thus, a library in the Python programming language was developed to extract data from the smaXtec system. The library retrieves data by using REST API technology.

Based on the data obtained and the purpose of the work (forecasting milk production), the most suitable methods are: "Learning with a teacher", exactly regression methods and forecasting methods.

According to the graph of the distribution of milk production by day (Figure 10), you can see useful data. 


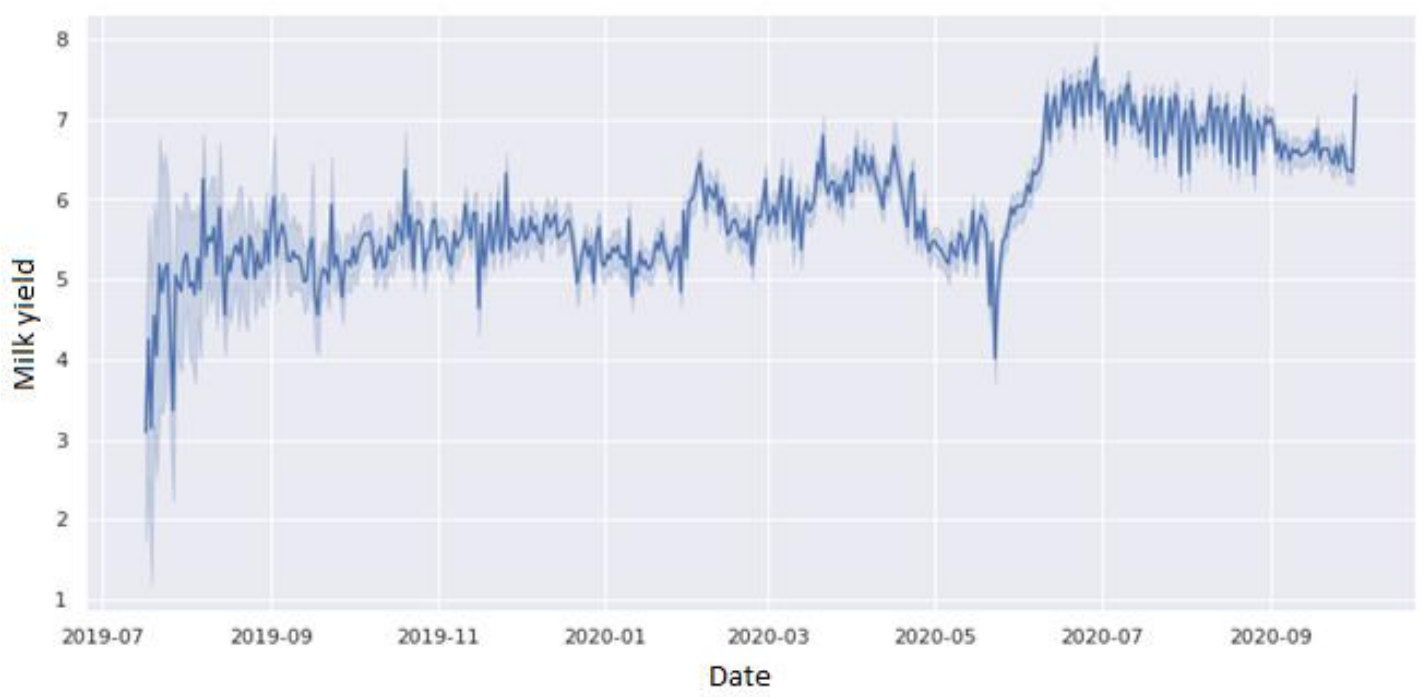

Figure 10: Distribution of milk yield sorted by days.

The stage of applying machine learning methods follows after the initial analysis and data preprocessing. Based on the data, the most suitable machine learning method is polynomial regression.

Figure 3 is a block diagram of the overall software architecture.

The software architecture consists of 3 main components:

1) System for remote monitoring of the condition of animals smaXtec;

2) Computing part (machine learning);

3) Interface for the farmer;

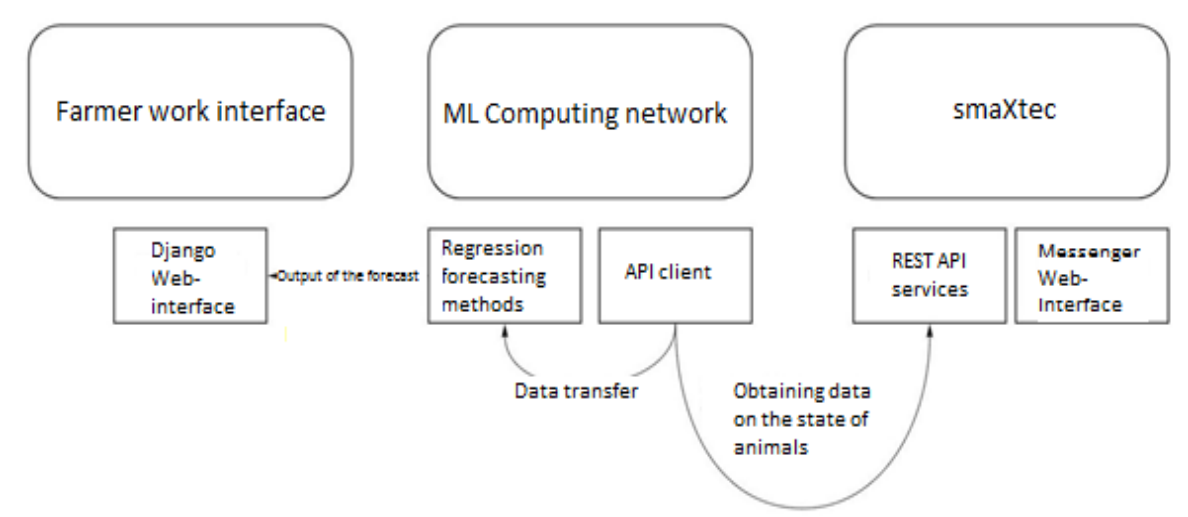

Figure 11: General architecture of the software.

A simple and straightforward architecture makes it faster to make any changes, as well as easier to integrate with other systems. 


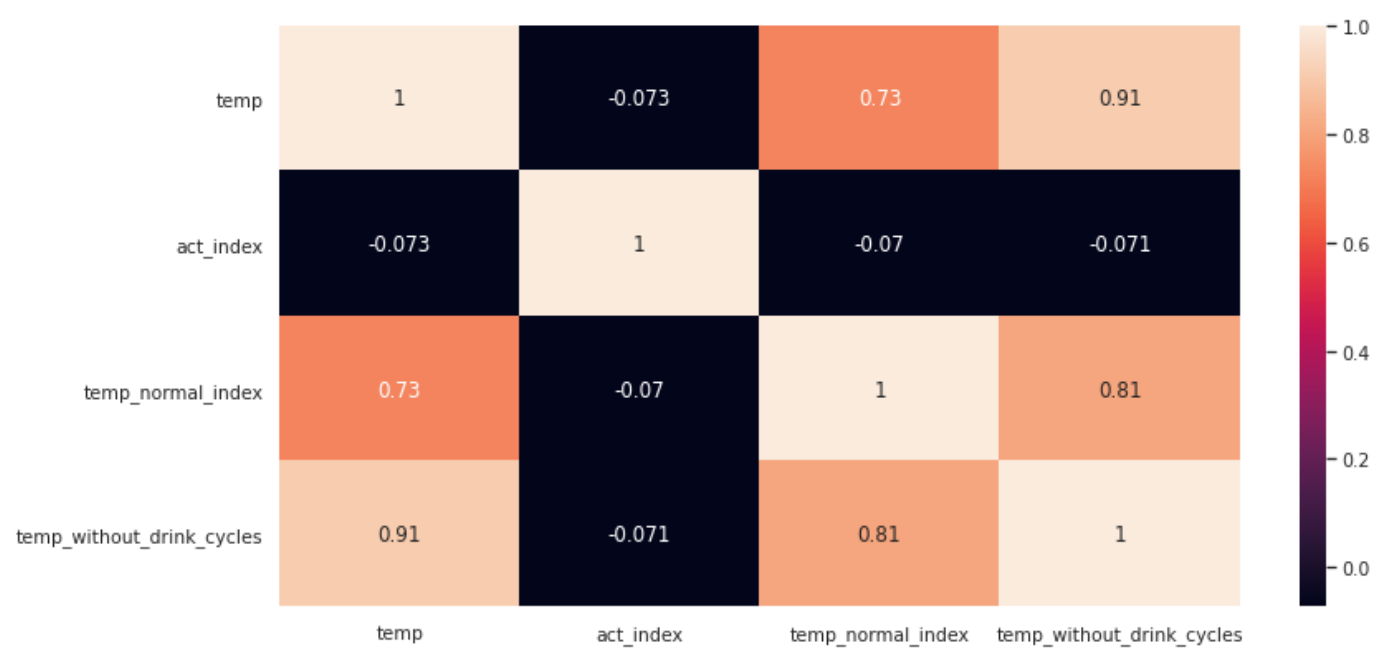

Figure 12: Correlation matrix of the obtained data.

As a result of visualization of the correlation matrix in the form of a heat map, presented in Figure 12, we concluded that there is no statistical relationship between such parameters as: body temperature, normal body temperature, body temperature without a drinking cycle, movement index. In this case, changes in the values of one or more of the above values do not accompany a systematic change in other values.

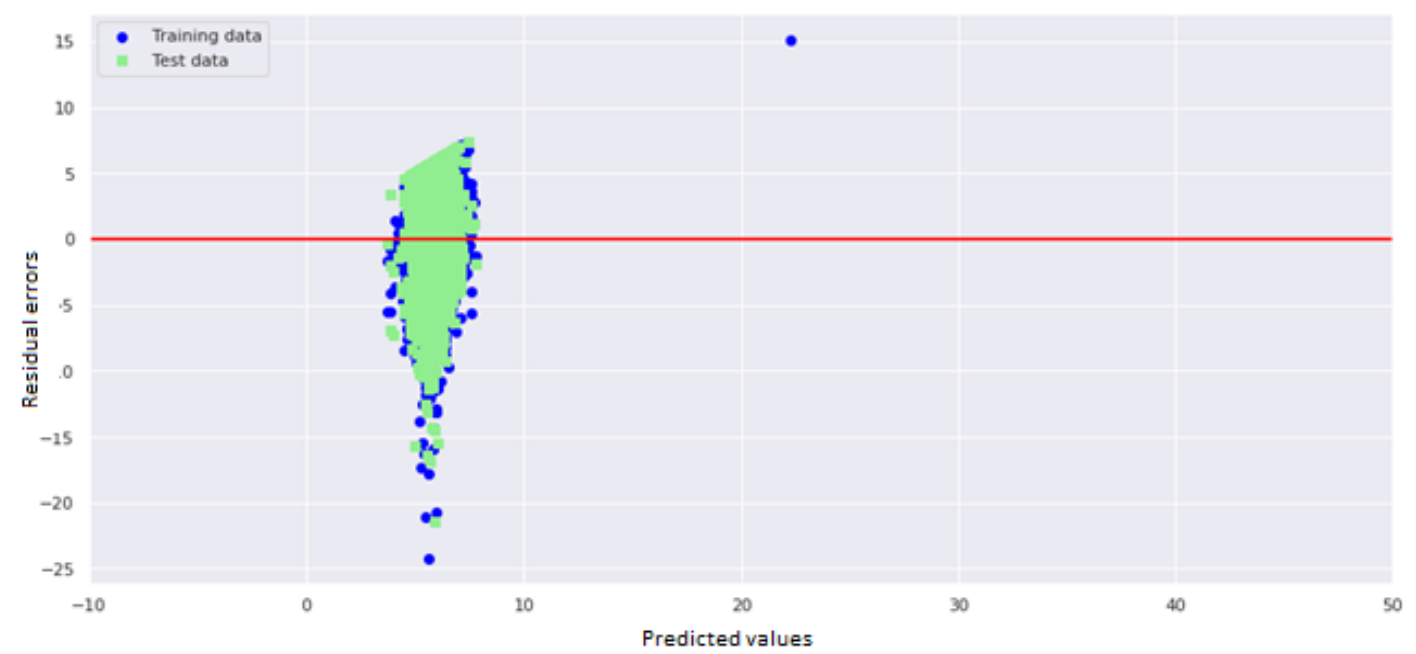

Figure 13: Regression analysis of the obtained data.

As a result of the conclusions made, we came to the need to use a simple linear regression for possible dependencies of the parameters of the initial data and the need to predict the volume of milk yield. In Figure 13, we see that the resulting errors do not have a normal distribution and the data variations around the regression line are not constant. Thus, prediction of the milk production of animals based on the above parameters (body temperature, normal body temperature, body temperature without a drinking cycle, movement index, milk yield) is also impossible.

Thus, we can conclude that the initial data (body temperature, normal body temperature, body temperature without a drinking cycle, movement index, milk yield) obtained from the smaXtec 
bolus system and data on the milk production of animals are not dependent. As a result, there is no need to use the initial data parameters for the effect on the volume of milk yield.

\section{Conclusions}

Upon analyzing the problem of cow diseases diagnostics, we came to the conclusion that it is necessary to develop an expert system of cattle diseases diagnostics. When setting the main tasks to build an expert system, the main one was to determine the input and output data of this system. By using the induction method, we have identified separate groups of input and output data, which will be used to build this system. The next stage was the creation of a generalized web architecture, with the indication of individual functional blocks and equally developed the basic scenario of the use of the intellectual system. When diagnosing a disease, the way the knowledge base is presented plays an important role, which in turn depends on the experience of team of veterinarians. Information on main symptoms and diseases has been collected through questionnaires and this information is structured and presented for better understanding. Thus, a model of knowledge representation has been developed, which leads to an accurate diagnosis. Together with a team of veterinarians, each symptom and symptom complex was given the weight coefficients required for a more accurate diagnosis of the disease.

Thus, we can conclude that the developed expert system for addressing veterinary medicine challenges is effective. By comparing the percentage ratios of the results of the questionnaire of two groups, it becomes obvious that its use is expedient. A detailed analysis of the test subjects' answers has been made and all regularities in both cases of testing have been taken into account. Conclusions were made that the process of diagnosing diseases is simplified in terms of speed of decision-making and their reliability, a direct correlation between the number of detected initial symptoms of the disease and the correct formulation of the diagnosis was revealed. Also, with the participation of veterinary students, an evaluation of the user interface was conducted, which included checking system design and correct compilation of the knowledge base to meet user requirements.

In summary, the developed software has shown its need for use. In future, the database of expert system on diseases and symptoms will be expanded, all deficiencies related to the convenience of the user interface and the operation of the program in general will be taken into account and eliminated.

An expert system under development provides information on 16 major infectious diseases and 103 symptoms, which is currently being developed and populated in the database. The development works are carried out in the S. Seifullin Kazakh Agro Technical University, at the faculties of computer systems and veterinary medicine.

In the future, work will also be done to integrate the system under development with existing animal control systems, to automate the processes of their interaction and data exchange. It is necessary to work with symptoms and weight values, that is, it is necessary to work on choosing an index of the significance of the manifestation of a symptom, instead of its usual manifestation or absence.

\section{ACKNOWLEDGEMENTS}

The authors would like to thanks for the veterinarian team expertise, Faculty of Veterinary Science, Kazakh Agro Technical University named after S. Seifullin. The work was carried out as 
part of the project "Transfer and adaptation of innovative technologies to optimize production processes at dairy farms in Northern Kazakhstan" (BR06349515).

\section{REFERENCES}

[1] E. Tullo, I. Fontana, D. Gottardo, K.H. Sloth, M. Guarino. Technical note: Validation of a commercial system for the continuous and automated monitoring of dairy cow activity// J.Dairy Sci. 99:7489-7494. http://dx.doi.org/10.3168/jds.2016-11014. American Dairy Science Association, 2016.

[2] Emre Aydemir, İnci Bilge. Automation Applications in Integrated Animal Production System. Turkish Journal of Agriculture - Food Science and Technology, 8(3): 643-644, 2020. DOI: https://doi.org/10.24925/turjaf.v8i3.643-644.3133.

[3] O. Shopagulov, I. Tretiakov, A. Ismailova, "An expert system for diagnosis cow diseases," Journal of Theoretical and Applied Information Technology, No 15, Vol.98., 2020, pp. 3106-3115.

[4] Ermekov Aidar. Meat March, 2013, [Online]. Available at: http://mkkz.kz/article/2013/02/11/810619-myasnoy-marsh.html/ (in Russian).

[5] Committee on Statistics of the Ministry of National Economy of the Republic of Kazakhstan: [Online]. Available at: http://www.kazagro.kz/analiticeskij-obzor-po-zivotnovodstvu/ (in Russian).

[6] The Committee on Statistics of the Ministry of National Economy of the Republic of Kazakhstan: [Online]. Available at: https://www.zakon.kz/4951625-obem-veterinarnyh-uslug-v-kazahstane.html/ (in Russian).

[7] L. I. Zubkova, "The effect of diseases of the udder on the milk productivity of cows," Dairy and beef cattle breeding, vol. 4, 2005, pp. 35-37. (in Russian).

[8] H. Qin, J. Xiao, X. Gao, H. Wang, "Horse-Expert: An aided expert system for diagnosing horse diseases," Veterinary Sciences, vol. 4, 2016, pp. 907-9015.

[9] M. Dorosh, Diseases of cattle, Veche, Moscow, 2007, 7 p. (in Russian).

[10] Yu. N. Kozlov, N. M. Kostomakhin, Genetics and selection of farm animals, Kolos, Moscow, 2013, 100 p. (in Russian).

[11] O. V. Zavyazkin, Breeding and keeping cattle, BAO, Kiev, 2012, 100p. (in Russian).

[12] Fu Zetian, Xu Feng, Zhou Yun, Zhang Xiao Shuan, "Pig-vet: a web-based expert system for pig disease diagnosis," Expert Systems with Applications, vol. 29, pp. 93-103, 2005.

[13] Daoliang Li, Zetian Fu, Yanqing Duan, "Fish-Expert: a web-based expert system for fish disease diagnosis," Expert Systems with Applications, vol. 23, pp. 311-320, 2002.

[14] Paolo Liberati, Paolo Zappavigza, "Improving the automated monitoring of dairy cows by integrating various data acquisition systems," Computers and electronics in agriculture, vol. 68, pp. 62-67, 2009.

[15] D. Rice, Common dog diseases and health problems 4-H Companion Animal Health, 2014, [Online]. Available at: https://www.extension.purdue.edu/extmedia/4H/4-H-852-W.pdf/

[16] E. B. Hunt, Artificial Intelligence, New York, San Francisco, London, Academic Press, 558 p, 1975.

[17] M. I. Makarov, V. M. Lokhin, Intelligent Automatic Control System, Moscow: Fizmatlit, 2001, 576 p. (in Russian).

[18] T. A. Gavrilova, V. F. Khoroshevsky, Knowledge Base of Intelligent Systems, St. Petersburg: Piter, 2000, 384 p. (in Russian).

[19] D. Zeldis and S. Prescott, "Fish disease diagnosis program - Problems and some solutions" Aquacultural Engineering, vol. 23, no. 1-3, 2000, pp. 3-11. 


\section{Authors}

Shopagulov Olzhas Almatovich, Doctoral student, Department of Information Systems, Kazakh Agro Technical University named after S.Seifullin, Nur-Sultan, Kazakhstan. In 2014 he graduated the Eurasian National University named after L.N. Gumilyov, received a bachelor's degree in radio engineering, electronics, telecommunications. Also in 2017, he graduated from S. Seifulin Agro Technical University with a radio engineering, electronics, telecommunications master's degree. He is project assistant on the development of an expert system for solving the problems of veterinary medicine (on the example of a dairy farm in Northern

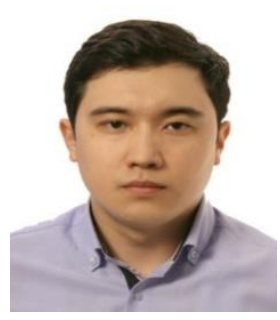
Kazakhstan). His research interests include the development of information systems, researches related to big data, as well as works related to data transfer.

Tretyakov Igor Igorevich recieved bachelor in technics and technologies from the Eurasian National University, Astana, Kazakhstan, in 2009. From 2009 to 2012, he was a software developer of various state information systems for electronic government. From 2012 to 2016 he was a software developer, data analyst and project manager for project in animal husbandry, animal recording in Kazakhstan. Participated in projects for animal genomic (gBLUP) and index evaluation in collaboration with INRA - French National Institute for Agricultural Research (dairy cattle) and Agricultural Business Research Institute, Australia (beef cattle). Since 2018, he is a lead of IT group in S.Seifullin Kazakh Agro Technical University in the "Transfer and

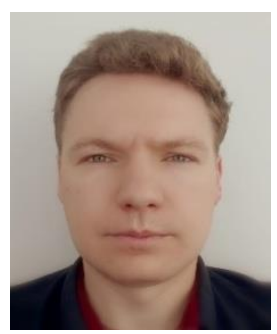
adaptation of innovative technologies to optimize production processes at dairy farms in Northern Kazakhstan" project. His interests include software development for web and mobile, automation systems and data analytics in agriculture.

Ismailova Aisulu Abzhapparovna, Doctor of Philosophy (PhD), Senior Lecturer. Received the bachelor degree in Physics and Mathematics from the Korkyt Ata Kyzylorda Pedagogical Institute, in 1993. Received the master's degree in Information Technology from the Eurasian National University named after L. N. Gumilyov, in 2006. From 2011 to 2014, she graduated a doctoral program at the Faculty of Information Technology at the Eurasian National University named after L.N. Gumilyov. His research interests are 3D modeling, working with spatial data, development of methods and software for creating information systems.

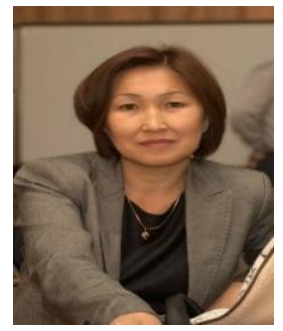

(C) 2021 By AIRCC Publishing Corporation. This article is published under the Creative Commons Attribution (CC BY) license. 


\title{
LOSSLESS STEGANOGRAPHY ON ORTHOGONAL VECTOR FOR 3D H.264 WITH LIMITED DISTORTION DIFFUSION
}

\author{
Juan Zhao ${ }^{1}$ and Zhitang $\mathrm{Li}^{2}$ \\ ${ }^{1}$ School of Mathematics \&Computer Science, \\ Wuhan Polytechnic University, Wuhan 430048, China \\ ${ }^{2}$ Institute of Computer Science and Technology, Huazhong University \\ of Science and Technology, Wuhan 430074, China
}

\begin{abstract}
In order to improve the undetectability, a lossless algorithm based on orthogonal vectors with limited distortion diffusion for 3D H.264 video is proposed in this paper. Inter-view distortion drift is avoided by embedding data into frames, which do not predict other views. Three conditions and pairs of coefficients are proposed to prevent intra-frame distortion diffusion. Several quantized discrete cosine transform coefficients are chosen from an embeddable luminance $4 \times 4$ block to construct a carrier vector, which is modified by an offset vector. When the carrier vector and the offset vector are orthogonal or near to be orthogonal, a data bit can be hidden. Experimental results indicate that the method is effective by enhancing peak signal-to-noise ratio with $7.5 \mathrm{~dB}$ and reducing the Kullback-Leibler divergence with 0.07 at least. More than $1.7 \times 10^{15}$ ways could be utilized for constructing the vectors, so it is more difficult for others to steal data.
\end{abstract}

\section{KEYWORDS}

Lossless Steganography, Reversible Data Hiding, Orthogonal Vector, 3D H.264, Distortion Drift

\section{INTRODUCTION}

The most significant requisite of steganography, which can be used to hide secret message into innocuous-looking media, is imperceptibility in covert communication. However, the host media will be distorted permanently if general steganography algorithms are used to hide information. Permanent damage is unbearable to medical images, military, law enforcement [1] and other sensitive fields. Therefore, lossless steganography (a kind of reversible data hiding)[2], videlicet reversible steganography or distortion-free steganography, which can restore the impaired video after extracting the secret information, has been a hot topic. Besides the sensitive fields, lossless steganography methods can also be employed in error concealment and other fields.

Histogram shifting (HS) [3-10] and difference expansion (DE) [11-15] are two principal lossless steganography approaches. In addition, integer pair swap (IPS) [16], pair-wise logical computation [17], and some other methods [18][19] have been employed to hide information reversibly. In typical HS algorithm [3], information is hidden into the peak points of a medium histogram. The number of embeddable data bits depends on the pixel number of the peak point in the histogram. The hierarchical relationships of original images are used, and the difference values between pixels are altered to hide data in [5]. All pixels are classified into wall pixels and non-wall pixels in [4], David C. Wyld et al. (Eds): SEAS, CMCA - 2021 
where interpolation and direction order are used for hiding data. In [6], the closest adjacent pixels are used to predict the visited pixel value and evaluate its just noticeable difference. Prediction-error shift is used to improve the embedding performance in [8, 9]. In [9], multiple pairs of expansion bins are utilized for each histogram , and the multiple-expansion-bin-selection for optimal embedding is formulated as an optimization problem.

In difference expansion method [11], the difference between two neighboring pixels is doubled to hide message. The secret information and a compressed location map are hidden into the difference values. To increase the payload, 16 bits are embedded into a $4 \times 4$ pixel block in a two-dimensional DE scheme [12].The host image is divided into non-overlapped equal-sized blocks in the high-fidelity technique [13] based on prediction-error expansion and pixel-value-ordering. Bidirectional difference expansion is used in [14] with three steps.

However, in traditional steganography algorithms, the selection space of embeddable location is small. How to improve the undetectability and security of lossless steganography algorithm is a key issue of covert communication. Video has so many frames that it can ensure adequate storage space. [20] Hence, through embedding a little information into a frame, we can guarantee video quality and improve the invisibility and undetectability. H.264 is the standard for video compression with high compression efficiency. 3D H.264 video is encoded or decoded through multi-view coding, which is an extension of H.264. At the encoder, intra-frame, inter-frame and inter-view predictions are used to compress the original YUV videos into a 3D H.264 video. In order to watch the video on the screen, the 3D H.264 video needs to be decompressed into YUV videos by intra-frame, inter-frame and inter-view predictions at the decoder. So if one block of a video is changed to embed data, the other blocks of the same frame, other frames or views in the corresponding YUV videos may also be modified, it is called intra-frame, inter-frame, or inter-view distortion drift [21], which is not considered in the literatures on $3 \mathrm{D}$ video data hiding [22-24].

In order to improve the undetectability and security of steganography, we present a novel lossless steganography algorithm based on orthogonal vector (If the inner product of two vectors is zero, it is called that the two vectors are orthogonal) for 3D H.264 video with limited distortion drift in this paper. Inter-view distortion propagation is avoided by embedding information into frames that do not predict other views. Embeddable blocks based on restrictive conditions are selected to prohibit intra-frame distortion shift. A carrier vector is composed of some quantized discrete cosine transform (QDCT) coefficients or coupling coefficients in one embeddable $4 \times 4$ block. An offset vector is set for recording the modification of the carrier vector. By dividing the inner product of the carrier vector and offset vector into several disjoint intervals, the information is hidden according to the interval of inner product. The carrier vector is not altered for hiding information 0 ; otherwise, the offset vector is added to or subtracted from the carrier vector according to the inner product of the two vectors. The receiver extracts information and restores the carrier according to the interval of inner product.

Compared with the current methods, the contributions of this paper are presented as follows. A. Three conditions and two sets of coupling coefficients are proposed for limiting intra-frame distortion drift. B. When the three conditions are used to avoid intra-frame distortion drift, over $1.7 \times 10^{15}$ ways of constructing the carrier vector and its offset vector increase the difficulty for others stealing data.

The rest of the paper is organized as follows. The way of avoiding distortion diffusion for 3D H.264 video is brought in Section 2. Section 3 describes the lossless steganography algorithm and Section 4 gives the experimental results. At last, the paper is concluded in Section 5. 


\section{Distortion Diffusion Prevention}

The original block denoted by $B^{O}$ in the original YUV video is processed by equation (1) at the 3D H.264 encoder.

$$
B^{O}-B^{P}=B^{R O}
$$

Where $B^{P}$ is the prediction block and $B^{R O}$ is the residual block. Undergoing discrete cosine transformation and quantization, the residual block $B^{R O}$ becomes a QDCT block (denoted by $Y$ ). Finally, YUV videos will be changed into 3D H.264 video by entropy encoding of QDCT blocks. Because this entropy encoding is a lossless compression process, at the decoder, the data embedded in some QDCT coefficients could be extracted completely after entropy decoding (lossless decompression). Undergoing the inverse quantization and inverse discrete cosine transform, the QDCT block $Y$ becomes a residual block denoted by $B^{R}$ (Cause discrete cosine transformation and quantization are loss compressions, $B^{R}$ is different from the original residual block $B^{R O}$ ), which will be added to the prediction block $B^{P}$ for reconstructing the video.

The prediction block $B^{P}$ of a block in a frame could be computed through inter-view prediction, inter-frame prediction, or intra-frame prediction. Horizontal inter-frame prediction and vertical inter-view prediction of a 3D H.264 video with hierarchical B coding and two views are illustrated in Figure 1[7]. There are 16 frames in one group of picture, where each view has eight frames. Only intra-frame prediction is used for $\mathrm{I}_{0}$ frame, so the distortion of other frames will not affect $\mathrm{I}_{0}$ frame. However, hiding data into $\mathrm{I}_{0}$ frame will sway all the frames in the two adjacent groups of pictures predicted by $\mathrm{I}_{0}$ frame. By contrast, embedding data into $\mathrm{P}_{0}$ frame will not lead to inter-view distortion drift because $\mathrm{P}_{0}$ frame in the right view does not predict frames in the left view. Furthermore, inter-view distortion drift and inter-frame distortion drift could be avoided by embedding data into $b_{4}$ frames. Therefore, compared with embedding data into $\mathrm{I}_{0}$ frames, better video quality can be achieved through embedding data into $\mathrm{P}_{0}$ or $\mathrm{b}_{4}$ frames. However, $\mathrm{b}_{4}$ frame is located at the lowest level and is easy to be discarded during the process of transmission in the network. Compared with $\mathrm{b}_{4}$ frame, better video quality cannot be acquired through embedding data into $\mathrm{P}_{0}$ frame. However, $\mathrm{P}_{0}$ frame is a key picture at the highest level, resulting that it cannot be lost easily during the process of the network transmission. Consequently, stronger robustness can be obtained by hiding data into $\mathrm{P}_{0}$ frame compared with $\mathrm{b}_{4}$ frame. Therefore, the best combination of video quality and robustness can be obtained by hiding data into $\mathrm{P}_{0}$ frame, so it is selected to embed information in this paper.

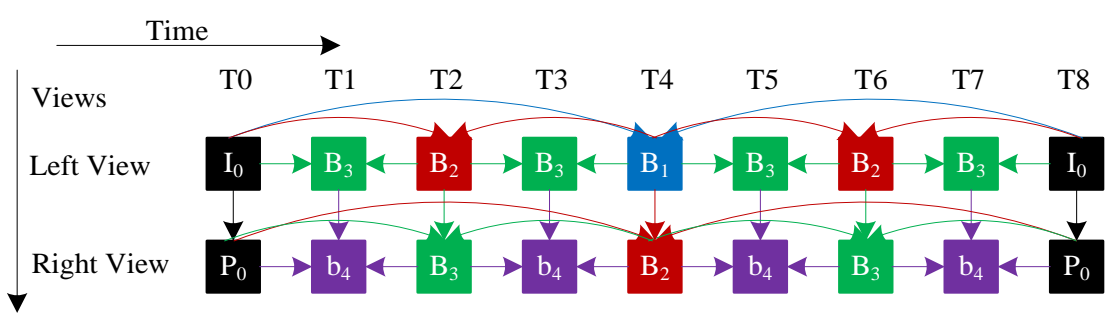

Figure 1. Prediction structure of3D H.264 with two views

At the encoder, through using inter-view prediction, inter-frame prediction, or intra-frame prediction, the prediction block $B^{P}$ of a block is achieved to compute its residual block $B^{R O}$, which will be changed into a QDCT block $Y$ by the $4 \times 4$ discrete cosine transform and quantization formulated as 


$$
Y=\operatorname{round}\left[\left(C_{f} \cdot B^{R O} \cdot C_{f}^{T}\right) \otimes\left(E_{f} / Q\right)\right]=\left[\begin{array}{cccc}
Y_{00} & Y_{01} & Y_{02} & Y_{03} \\
Y_{10} & Y_{11} & Y_{12} & Y_{13} \\
Y_{20} & Y_{21} & Y_{22} & Y_{23} \\
Y_{30} & Y_{31} & Y_{32} & Y_{33}
\end{array}\right],
$$

where $\mathrm{Q}$ is the quantization step size, $\otimes$ is a math operator, by which each element in the former matrix is multiplied by the value at the corresponding position in the latter matrix,

$$
C_{f}=\left[\begin{array}{cccc}
1 & 1 & 1 & 1 \\
2 & 1 & -1 & -2 \\
1 & -1 & -1 & 1 \\
1 & -2 & 2 & -1
\end{array}\right], \quad E_{f}=\left[\begin{array}{cccc}
a^{2} & a b / 2 & a^{2} & a b / 2 \\
a b / 2 & b^{2} / 4 & a b / 2 & b^{2} / 4 \\
a^{2} & a b / 2 & a^{2} & a b / 2 \\
a b / 2 & b^{2} / 4 & a b / 2 & b^{2} / 4
\end{array}\right], a=1 / 2, b=\sqrt{2 / 5}
$$

When a data bit is hidden into one QDCT block $Y$ by modifying some QDCT coefficients, the QDCT block after embedding data is denoted by $Y^{E m b}$. Let $\Delta Y$ denote the modification caused by hiding information, it can be computed as

$$
\Delta Y=Y^{E m b}-Y .
$$

At the decoder, the residual block acquired by inverse quantization and $4 \times 4$ inverse discrete cosine transform is denoted by $B^{R}$, which can be calculated by

$$
B^{R}=\operatorname{round}\left[C_{d}^{T} \cdot\left(Y \otimes E_{d}\right) \cdot C_{d}\right],
$$

where $C_{d}=\left[\begin{array}{cccc}1 & 1 & 1 & 1 \\ 1 & 1 / 2 & -1 / 2 & -1 \\ 1 & -1 & -1 & 1 \\ 1 / 2 & -1 & 1 & -1 / 2\end{array}\right], \quad E_{d}=\left[\begin{array}{cccc}a^{2} & a b & a^{2} & a b \\ a b & b^{2} & a b & b^{2} \\ a^{2} & a b & a^{2} & a b \\ a b & b^{2} & a b & b^{2}\end{array}\right]$.

When a data bit is embedded through modifying some QDCT coefficients of one block, the residual block after embedding data is denoted by $B^{R E m b}$. Let $\Delta B^{R}$ depress the variation of the residual block between before and after hiding information. It can be computed as

$$
\Delta B^{R}=B^{R E m b}-B^{R}=\operatorname{round}\left[C_{d}^{T} \cdot\left(\Delta Y \cdot Q \otimes E_{d}\right) \cdot C_{d}\right] .
$$

Take the QDCT coefficient $Y_{32}$ as an example to explain the distortion caused by hiding data. Suppose $r$ is added to $Y_{32}$, the modification of the QDCT block for hiding data is $\Delta Y=\left[\begin{array}{llll}0 & 0 & 0 & 0 \\ 0 & 0 & 0 & 0 \\ 0 & 0 & 0 & 0 \\ 0 & 0 & r & 0\end{array}\right]$, then the alteration of the corresponding block in YUV video is

$$
\Delta B^{R}=\frac{1}{2} Q a b r\left[\begin{array}{cccc}
1 & -1 & -1 & 1 \\
-2 & 2 & 2 & -2 \\
2 & -2 & -2 & 2 \\
-1 & 1 & 1 & -1
\end{array}\right] .
$$


Similarly, changing any one other QDCT coefficient in a $4 \times 4$ block will cause the variation of the whole block in the corresponding YUV video. In the same way, for an $8 \times 8$ block, modifying one QDCT coefficient will alter the whole $8 \times 8$ block, whose affected region is bigger than that of the $4 \times 4$ block. In addition, only two kinds of transformations, $4 \times 4$ transformation and $8 \times 8$ transformation, are used in $3 \mathrm{D}$ H.264 standard. Hence, the $4 \times 4$ transform block is selected to embed information in this paper.

It can be inferred that the edge pixels denoted by $c_{0} \ldots c_{12}$ (shown in Figure 2) may be changed by hiding data into some QDCT coefficients of the blocks $B_{i, j-1}$ (the position of a block is expressed by $I$ and $j), B_{i-1, j-1}, B_{i-1, j}$, and $B_{i-1, j+1}$. On one hand, when inter-view prediction or inter-frame prediction is employed to compute the prediction block denoted by $B_{i, j}^{P}$, the block $B_{i, j}$ will not be affected by the change of $c_{0} \ldots c_{12}$, because $B^{P_{i, j}}$ is calculated by referring other frames. On the other hand, when intra-frame prediction is used by the current block $B_{i, j}$, its prediction block $B_{i, j}^{P}$ will be reckoned by the pixels $c_{0} \ldots c_{12}$. Therefore, the hiding induced deviation of the blocks $B_{i, j-1}, B_{i-1, j-1}, B_{i-1, j}$, and $B_{i-1, j+1}$ will propagate to the block $B_{i, j}$. This is called as intra-frame distortion drift. However, according to the intra-frame prediction modes shown in Figure 2, it can be seen that the block $B_{i, j}$ is not affected by the thirteen pixels $c_{0} \ldots c_{12}$ at the same time. For instance, when intra-frame prediction mode 0 is used by the current block $B_{i, j}$, only pixels $c_{1}, c_{2}, c_{3}$, and $c_{4}$ are used to predict the block $B_{i, j}$, so the distortion of the blocks $B_{i, j-1}, B_{i-1, j-1}$, and $B_{i-1, j+1}$ will not drift to the block $B_{i, j}$. Similarly, we can conceive the influence of $B_{i, j}$ over $B_{i, j+1}, B_{i+1, j+1}, B_{i+1, j}$, and $B_{i+1, j-1}$. Therefore, some conditions could be used to prevent intra-frame distortion drift.

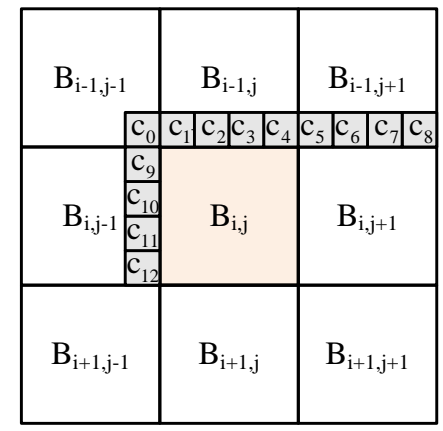

(a)

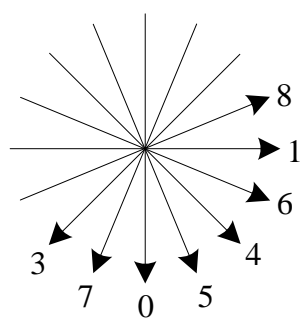

(b)

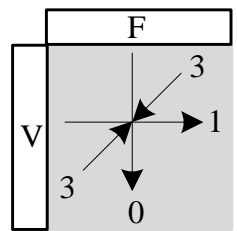

(c)

Figure 2. Intra-frame prediction mode (a) block position (b) the predictive direction of $4 \times 4$ and $8 \times 8$ luma block.(c) the predictive direction of $16 \times 16$ luma block.( In mode 2, all elements are predicted with the average of upper pixels denoted by $\mathrm{F}$ and left pixels denoted by $\mathrm{V}$, i.e. Mean $(\mathrm{F}+\mathrm{V})$ )

The prediction mode of intra-frame is denoted by prediction Mode. The mode type of macro block (MB) is denoted by mb_type. Let $p$ be the $m b \_t y p e$ of inter-view or inter-frame prediction. If the mb_type of the block $B_{i, j+1}$ is $p$, the prediction block $B_{i, j+1}^{P_{i}}$ is calculated by referring another frame, so the current block $B_{i, j}$ will not predict the block $B_{i, j+1}$. Otherwise, when the intra-frame prediction modes 0,3, 7 indicated in Figure 2(b), or 0 in Figure 2 (c) are used by the block $B_{i, j+1}$, it can be seen from the predictive directions that the block $B_{i, j+1}$ will not be predicted by the current block $B_{i, j}$. Therefore, if information is embedded into the QDCT coefficients of the current block $B_{i, j}$, whose right adjacent block $B_{i, j+1}$ meets Condition 1, the evoked distortion will not drift to its adjacent block $B_{i, j+1}$.

Condition 1. $\quad\left(\right.$ mb_type $\left._{B_{i, j+1}}=p\right) \cup\left[\right.$ predictionMode $\left.e_{B_{i, j+1}} \in\{0,3,7\}_{(4 \times 4 \text { or } 8 \times 8)} \cup\{0\}_{(16 \times 16)}\right]$

If the $m b \_t y p e$ of the block $B_{i+1, j}$ is $p$, the prediction block $B^{P_{i+1, j}}$ is not calculated by referring 
the current block $B_{i, j .}$ Otherwise, when the intra-frame prediction modes 1 and 8 indicated in Figure 2(b), or 1 in Figure 2 (c) are used by the block $B_{i+1, j}$, it can be seen from the predictive directions that the block $B_{i+1, j}$ will not be predicted by the current block $B_{i, j .}$. Similarly, when the $m b_{-}$type of the block $B_{i+1, j-1}$ is $p$, or the intra-frame prediction modes $0,1,2,4,5,6$, and 8 indicated in Figure 2(b), or 0, 1, 2, and 3 in Figure 2 (c) are used by the block $B_{i+1, j-1}$, the block $B_{i+1, j-1}$ will not be predicted by the current block $B_{i, j}$. Therefore, if information is embedded into the QDCT coefficients of the current block $B_{i, j}$, whose adjacent blocks $B_{i+1, j}$ and $B_{i+1, j-1}$ meet Condition 2, the evoked distortion will not drift to its adjacent blocks $B_{i+1, j}$ and $B_{i+1, j-1}$.

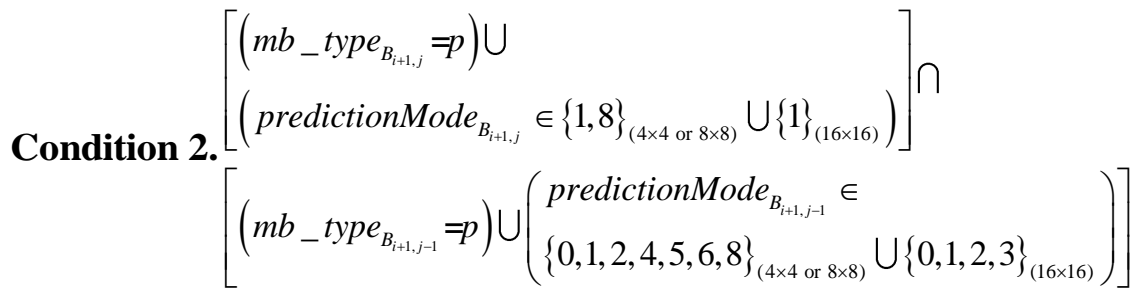

If the mb_type of the block $B_{i+1, j+1}$ is $p$, the prediction block $B^{P}{ }_{i+1, j+1}$ is not calculated by referring the current block $B_{i, j}$. Otherwise, when the intra-frame prediction modes $0,1,2,3,7$, and 8 indicated in Figure 2(b), or 0, 1, 2, and 3 in Figure 2(c) are used by the block $B_{i+1, j+1}$, it can be seen from the predictive directions that the block $B_{i+1, j+1}$ will not be predicted by the current block $B_{i, j}$. Therefore, if data is embedded into the QDCT coefficients of the block $B_{i, j}$, whose adjacent block $B_{i+1, j+1}$ meets Condition 3, the evoked distortion will not drift to its adjacent block $B_{i+1, j+1}$.

Condition 3. $\left(\right.$ mb $_{-}$type $\left._{B_{i+1, j+1}}=p\right) \cup\left[\right.$ predictionMode $\left._{B_{i+1, j+1}} \in\{0,1,2,3,7,8\}_{(4 \times 4 \text { or } 8 \times 8)} \cup\{0,1,2,3\}_{(16 \times 16)}\right]$

It is obvious that if information is hidden into the QDCT coefficients of the current block $B_{i, j}$, whose adjacent blocks meet Condition 1, Condition 2, and Condition 3, the evoked distortion will not drift to any neighboring blocks. Therefore, these three conditions could be used for hiding data without intra-frame distortion drift. When Condition 1, Condition 2, and Condition 3 could not be satisfied at the same time, intra-frame distortion drift could be prevented by compensation method. When data is hidden into some QDCT coefficients of the current block $B_{i, j}$, suppose the alternation of the QDCT block is $\Delta Y=\left[\begin{array}{cccc}0 & 0 & 0 & 0 \\ 0 & 0 & 0 & 0 \\ 0 & 0 & 0 & 0 \\ -r & 0 & r & 0\end{array}\right]$, then the alteration of the corresponding block in YUV video is

$$
\Delta B^{R}=Q a b r\left[\begin{array}{cccc}
0 & -1 & -1 & 0 \\
0 & 2 & 2 & 0 \\
0 & -2 & -2 & 0 \\
0 & 1 & 1 & 0
\end{array}\right]
$$

When the values of QDCT coefficients $Y_{30}$ and $Y_{32}$ are changed at the same time (if $r$ is added to $Y_{32}, r$ should be subtracted from $Y_{30}$ correspondingly), values on the last column of matrix $\Delta B^{R}$ are zero. It shows that pixels on the last column of the current block $B_{i, j}$ are not altered by hiding data. Because pixels on the last column may predict the neighboring blocks $B_{i, j+1}$ or $B_{i+1, j+1}$, so the distortion of the current block $B_{i, j}$ will not infect the two blocks. Accordingly, the pair of coefficients $\left(Y_{30}, Y_{32}\right)$ could be coupled to confine intra-frame distortion drift partly. When data is hidden into some QDCT 
coefficients of the current block $B_{i, j}$, assume the alternation of the QDCT block is ${ }_{\Delta Y}=\left[\begin{array}{cccc}0 & 0 & 0 & 0 \\ 0 & 0 & 0 & r \\ 0 & 0 & 0 & 0 \\ 0 & 0 & 0 & -2 r\end{array}\right]$, then the alteration of the corresponding block in YUV video is

$$
\Delta B^{R}=\frac{5}{4} Q b^{2} r\left[\begin{array}{cccc}
0 & 0 & 0 & 0 \\
1 & -2 & 2 & -1 \\
-1 & 2 & -2 & 1 \\
0 & 0 & 0 & 0
\end{array}\right] \text {. }
$$

When the values of QDCT coefficients $Y_{13}$ and $Y_{33}$ are changed at the same time (if $r$ is added to $Y_{13}$, $2 r$ should be subtracted from $Y_{33}$ correspondingly), the values on the bottom row of matrix $\Delta B^{R}$ are zero. It shows that pixels on the bottom row of the current block $B_{i, j}$ are not changed by hiding data. Because pixels on the last column may predict the neighboring blocks $B_{i+1, j}, B_{i+1, j-1}$ or $B_{i+1, j+1}$, so the distortion of the current block $B_{i, j}$ will not infect the three blocks. Accordingly, the pair of coefficients $\left(Y_{13}, 2 Y_{33}\right)$ could be coupled to prevent intra-frame distortion drift partly. Similarly, we can get two sets of coupling coefficients, which are denoted by $C_{\text {set }}$ and $R_{\text {set }}$.

$$
\begin{aligned}
& C_{\text {set }}=\left\{\left(Y_{00}, Y_{02}\right),\left(Y_{10}, Y_{12}\right),\left(Y_{20}, Y_{22}\right),\left(Y_{30}, Y_{32}\right),\left(Y_{01}, 2 Y_{03}\right),\left(Y_{11}, 2 Y_{13}\right),\left(Y_{21}, 2 Y_{23}\right),\left(Y_{31}, 2 Y_{33}\right)\right\} \\
& R_{\text {set }}=\left\{\left(Y_{00}, Y_{20}\right),\left(Y_{01}, Y_{21}\right),\left(Y_{02}, Y_{22}\right),\left(Y_{03}, Y_{23}\right),\left(Y_{10}, 2 Y_{30}\right),\left(Y_{11}, 2 Y_{31}\right),\left(Y_{12}, 2 Y_{32}\right),\left(Y_{13}, 2 Y_{33}\right)\right\}
\end{aligned}
$$

When data is hidden into any coupling coefficients in $C_{\text {set }}$ (if $r$ is added to the former coefficient, $r$ or $2 r$ should be subtracted from the latter coefficient correspondingly), the values on the rightmost column of matrix $\Delta B^{R}$ are 0 , so the distortion of the block $B_{i, j}$ will not propagate its neighboring blocks $B_{i, j+1}$ and $B_{i+1, j+1}$. Hiding data into any coupling coefficients in $R_{s e t}$, we can make the values at the bottom row of matrix $\Delta B^{R}$ be zero, so the distortion of block $B_{i, j}$ will not affect its adjacent blocks $B_{i+1, j}, B_{i+1, j-1}$, and $B_{i+1, j+1}$. It can be seen that the coupling coefficients in $R_{s e t}$ could be combined with Condition 1 to avoid intra-frame distortion drift, and the coupling coefficients in $C_{s e t}$ can be combined with Condition 2 to eliminate intra-frame distortion drift. For instance, when the adjacent blocks of the block $B_{i, j}$ do not satisfy Condition 1 to 3 at the same time, if Condition 1 is satisfied by the adjacent block $B_{i, j+1}$, it will not be affected by the block $B_{i, j}$. In addition, if coupling coefficients such as $\left(Y_{02}, Y_{22}\right)$ of $R_{\text {set }}$ are selected from the block $B_{i, j}$ for hiding data, the distortion of the block $B_{i, j}$ will not drift to its neighboring blocks $B_{i+1, j}, B_{i+1, j-1}$, and $B_{i+1, j+1}$.

\section{LOSSLESS ALgORITHM BASED ON ORTHOGONAL VECTOR}

The presented lossless steganography algorithm based on orthogonal vector for 3D H.264 video is depicted in Figure 3. At first, the information to be hidden is encrypted, and the 3D H.264 video is entropy decoded to gain the QDCT coefficients and intra-frame prediction modes.

\subsection{Information Embedding}

Denote the threshold as $H$. We select a $4 \times 4$ luminance QDCT block of $\mathrm{P}_{0}$ frame in the right view according to $\left|Y_{00}\right| \geq H$ (threshold $H=0,1,2, \ldots$. The bigger the threshold $\mathrm{H}$ is, the fewer embeddable blocks will be found, and the less the distortion will be. Compared with a block with small $\left|Y_{00}\right|$, the distortion caused by hiding data into a block with big $\left|Y_{00}\right|$ is less. ). If Conditions 1 to 3 are satisfied at the same time, the current block is chosen as an embeddable block. In the $4 \times 4$ block, a QDCT coefficient could be changed for hiding data, whereas only 16 selections can be used. If the third party identifies the marked block and the steganography algorithm, the probability for calculating the hidden data bit directly is $1 / 16=0.0625$. In order to reduce the probability of being cracked, we 
embed data by choosing $n$ QDCT coefficients from the block to make up a carrier vector denoted by $\psi=\left(x_{1}, x_{2}, \ldots, x_{i}, \ldots, x_{n}\right)(n \in[2,16])$ such as $\psi=\left(Y_{22}, Y_{32}\right)=(2,0)$. Denote the carrier vector after hiding data as $\psi^{\prime}=\left(x_{1}^{\prime}, x_{2}^{\prime}, \ldots, x_{i}^{\prime}, \ldots, x_{n}^{\prime}\right)$. In order to express the size of the modified value on carrier vector for embedding data reversibly, we construct a non zero offset vector denoted as $\partial=\left(z_{1}, z_{2}, \ldots, z_{i}, \ldots, z_{n}\right)$ such as $\partial=(0,1)$. Let $\varphi$ be the included angle from the carrier vector $\psi$ to the offset vector $\partial$, and $\varphi^{\prime}$ be the included angle from $\psi^{\prime}$ to $\partial$. Denote the length of the carrier vector as $|\psi|=\left(x_{1}^{2}+x_{2}^{2}+\ldots+x_{n}^{2}\right)^{1 / 2}$. If the length $|\psi|$ or the included angle $\varphi$ is changed for hiding data, more computations need to be done since the modification of QDCT coefficient must be computed. So we hide data by changing the direction of the carrier vector, as shown in Figure 4 and (9), where the value of QDCT coefficient is changed directly and simply.

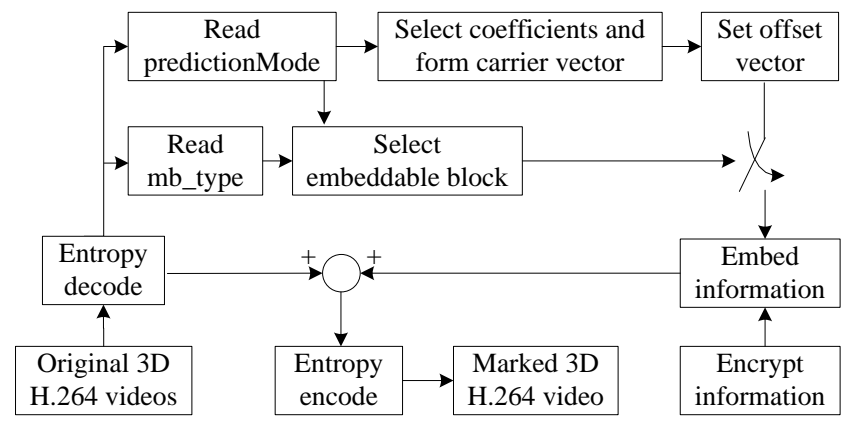

(a)

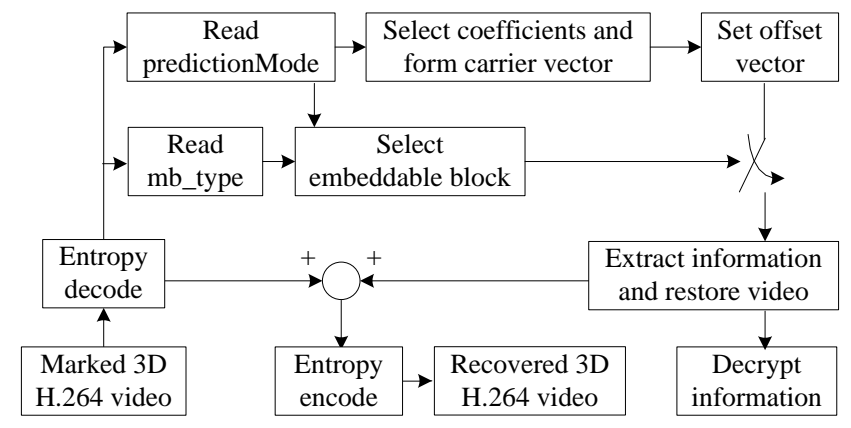

(b)

Figure 3. The flowchart of presented algorithm. (a) Embedding. (b) Extraction

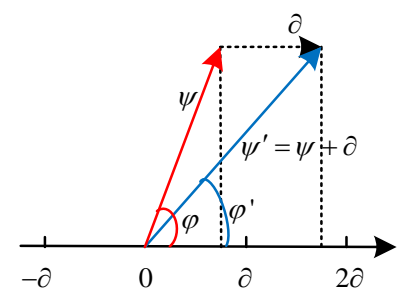

(a) $\psi \bullet \partial \geq 0$

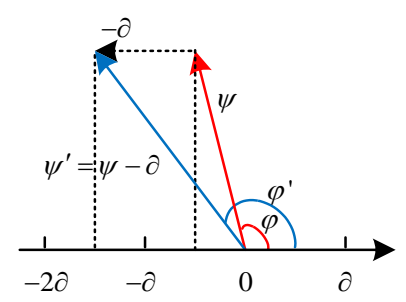

(b) $\psi \bullet \partial<0$

Figure 4. The modification of carrier vector $\psi$

Denote a bit of information as $u \in\{0,1\}[10]$. If the information $u$ is 0 , the carrier vector is not changed. Otherwise, in order to embed data reversibly, the carrier vector is modified.

$$
\psi^{\prime}=\left\{\begin{array}{l}
\psi+\partial, \text { if }(\psi \bullet \partial \geq 0) \\
\psi-\partial, \text { if }(\psi \bullet \partial<0)
\end{array} \Leftrightarrow x_{i}^{\prime}=\left\{\begin{array}{l}
x_{i}+z_{i}, \text { if }(\psi \bullet \partial \geq 0) \\
x_{i}-z_{i}, \text { if }(\psi \bullet \partial<0)
\end{array}\right.\right.
$$


Where $\psi \bullet \partial\left(=x_{1} z_{1}+x_{2} z_{2}+\ldots+x_{n} z_{n}=|\psi||\partial| \cos \varphi\right)$ is the inner product of $\psi$ and $\partial$.

In order to get minimum offset $|\partial|$, we could set only one $z_{i}$ in $\partial$ is 1 or -1 and the others are 0 . Nonzero $z_{i}$ represents the embedding position and the modified value of the embedding coefficient $x_{i}$.

Proposition. If $|\psi \bullet \partial|<\partial \bullet \partial$, then a bit of information $u$ could be hidden reversibly.

Proof. If $\psi \bullet \partial=0$, then $\psi$ and $\partial$ are orthogonal, denoted as $\psi \perp \partial$. At this time, $\cos \varphi=0$. So we can infer that $\lim _{\cos \varphi \rightarrow 0}(\psi \bullet \partial)=0$.

Suppose $|\psi \bullet \partial|=\partial \bullet \partial$, we can reason that

$$
|\psi \bullet \partial|=\partial \bullet \partial \Leftrightarrow\left\{\begin{array} { c } 
{ \psi \bullet \partial = \partial \bullet \partial } \\
{ \psi \bullet \partial = - \partial \bullet \partial }
\end{array} \Leftrightarrow \left\{\begin{array}{l}
(\psi-\partial) \bullet \partial=0 \\
(\psi+\partial) \bullet \partial=0
\end{array}\right.\right.
$$

All points in the space from the flat $(\psi-\partial) \bullet \partial=0$ to $(\psi+\partial) \bullet \partial=0$ could be used for hiding message. Hence our embedding condition could be

$$
|\psi \bullet \partial|<\partial \bullet \partial \Leftrightarrow|\psi||\partial||\cos \varphi|<|\partial||\partial| \Leftrightarrow|\psi||\cos \varphi|<|\partial| .
$$

(11) shows that information could be hidden when the projection $|\psi||\cos \varphi|$ of the carrier vector $\psi$ on the offset vector $\partial$ is less than the length of $\partial$. When the information $u$ is 0 , the carrier is not changed, so the projection $\left|\psi^{\prime}\right| \cos \varphi^{\prime} \in(-|\partial|,|\partial|)$. In order to hide information 1 , the carrier vector $\psi$ is altered as shown in (9) and Figure 4, the projection $|\psi| \cos \varphi \in[0,|\partial|)$ is turned into $\left|\psi^{\prime}\right| \cos \varphi^{\prime} \in[|\partial|, 2|\partial|)$ by adding the offset vector $\partial$ to the carrier vector $\psi$. The projection $|\psi| \cos \varphi \in(-|\partial|, 0)$ is changed into $\left|\psi^{\prime}\right| \cos \varphi^{\prime} \in(-2|\partial|,-|\partial|)$ by subtracting the offset vector $\partial$ from the carrier vector $\psi$. When the value of $|\psi||\cos \varphi|$ belongs to the interval $[|\partial|, \infty)$ or $(-\infty,-|\partial|]$, message could not be hidden. In order to distinguish the interval of information 1 , the interval $[|\partial|, \infty)$ is changed to be $[2|\partial|, \infty)$ by adding the offset vector $\partial$ to the carrier vector $\psi$, and the interval $(-\infty,-|\partial|]$ is changed to be $(-\infty,-2|\partial|]$. Then we could extract information and recover the carrier vector according to different intervals of $\left|\psi^{\prime}\right| \cos \varphi^{\prime}$. Therefore, this proposition is proved.

The carrier vector $\psi$ is changed for embedding data as shown in Figure 4 and (12). Information 0 and 1 are hidden into different intervals based on the value of $|\psi| \cos \varphi$. According to (11), we can infer the equivalent relations and the real embedding process (13), a corresponding version of (12). 


$$
\begin{aligned}
& \text { if }\{(|\psi||\cos \varphi|<|\partial|) \wedge(u=0)\} \\
& \psi^{\prime}=\psi \Rightarrow\left|\psi^{\prime}\right|\left|\cos \varphi^{\prime}\right|<|\partial| \\
& \text { else if }\{(0 \leq|\psi| \cos \varphi<|\partial|) \wedge(u=1)\} \\
& \psi^{\prime}=\psi+\partial \Rightarrow|\partial| \leq\left|\psi^{\prime}\right| \cos \varphi^{\prime}<2|\partial| \\
& \text { else if }\{(-|\partial|<|\psi| \cos \varphi<0) \wedge(u=1)\} \\
& \psi^{\prime}=\psi-\partial \Rightarrow-2|\partial|<\left|\psi^{\prime}\right| \cos \varphi^{\prime}<-|\partial| \\
& \text { else if }(|\psi| \cos \varphi \geq|\partial|) \\
& \psi^{\prime}=\psi+\partial \Rightarrow\left|\psi^{\prime}\right| \cos \varphi^{\prime} \geq 2|\partial| \\
& \text { else if }(|\psi| \cos \varphi \leq-|\partial|) \\
& \psi^{\prime}=\psi-\partial \Rightarrow\left|\psi^{\prime}\right| \cos \varphi^{\prime} \leq-2|\partial|
\end{aligned}
$$

When a $4 \times 4$ luminance QDCT block $B_{i, j}$ in $\mathrm{P}_{0}$ frame meets $\left|Y_{00}\right| \geq H$, Condition 1 is satisfied by its right adjacent block $B_{i, j+1}$, but Condition $\mathbf{2}$ or $\mathbf{3}$ is not satisfied, several coupling coefficients could be chosen from $R_{\text {set }}$ to combine with Condition 1 to prevent intra-frame distortion diffusion. If a $4 \times 4$ block in $\mathrm{P}_{0}$ frame satisfies $\left|Y_{00}\right| \geq H$, Condition 2 is satisfied by its adjacent blocks $B_{i+1, j}$ and $B_{i+1, j-1}$, but Condition 1 is not satisfied, some coupling coefficients in $C_{s e t}$ could be managed to unite with Condition 2 for removing distortion propagation.

$$
\begin{aligned}
& \text { if }(|\psi \bullet \partial|<\partial \bullet \partial) \wedge(u=0) \\
& \psi^{\prime}=\psi \Rightarrow\left|\psi^{\prime} \bullet \partial\right|<\partial \bullet \partial . \\
& \text { else if }(0 \leq \psi \bullet \partial<\partial \bullet \partial) \wedge(u=1) \\
& \psi^{\prime}=\psi+\partial \Rightarrow \partial \bullet \partial \leq \psi^{\prime} \bullet \partial<2 \partial \bullet \partial . \\
& \text { else if }(-\partial \bullet \partial<\psi \bullet \partial<0) \wedge(u=1) \\
& \psi^{\prime}=\psi-\partial \Rightarrow-2 \partial \bullet \partial<\psi^{\prime} \bullet \partial<-\partial \bullet \partial . \\
& \text { else if }(\psi \bullet \partial \geq \partial \bullet \partial) \\
& \psi^{\prime}=\psi+\partial \Rightarrow \psi^{\prime} \bullet \partial \geq 2 \partial \bullet \partial . \\
& \text { else if }(\psi \bullet \partial \leq-\partial \bullet \partial) \\
& \psi^{\prime}=\psi-\partial \Rightarrow \psi^{\prime} \bullet \partial \leq-2 \partial \bullet \partial .
\end{aligned}
$$

Coupling coefficients denoted as $\left(q_{1}, y_{1}\right),\left(q_{2}, y_{2}\right), \ldots,\left(q_{S}, y_{S}\right)(S \in[2,8])$ are divided into embedding coefficients and compensation coefficients. The former can compose an embedding carrier vector denoted as $\psi_{c}=\left(q_{1}, q_{2}, \ldots, q_{i}, \ldots, q_{S}\right)$. The latter could form a compensation vector denoted as $\omega=\left(y_{1}, y_{2}, \ldots, y_{i}, \ldots, y_{S}\right)$. We construct a non zero offset vector denoted as $\partial_{c}=\left(e_{1}\right.$, $\left.e_{2}, \ldots, e_{i}, \ldots, e_{S}\right)$. Denote the carrier vector after hiding data as $\psi_{c}^{\prime}=\left(q_{1}^{\prime}, q_{2}^{\prime}, \ldots, q_{i}^{\prime}, \ldots, q_{S}^{\prime}\right)$, and the compensation vector after hiding data as $\omega^{\prime}=\left(y_{1}^{\prime}, y_{2}^{\prime}, \ldots, y_{i}^{\prime}, \ldots, y_{S}^{\prime}\right)$. When the carrier vector $\psi_{\mathrm{c}}$ is changed, the compensation vector $\omega$ is modified accordingly.

\subsection{Information Extraction and Video Recovery}

After the 3D H.264 video is entropy decoded, if $\left|Y_{00}\right| \geq H$ and Conditions 1 to 3 are all satisfied, the current $4 \times 4$ QDCT block is chosen as an embeddable block, the process of information extraction and video recovery is exhibited in (14). 


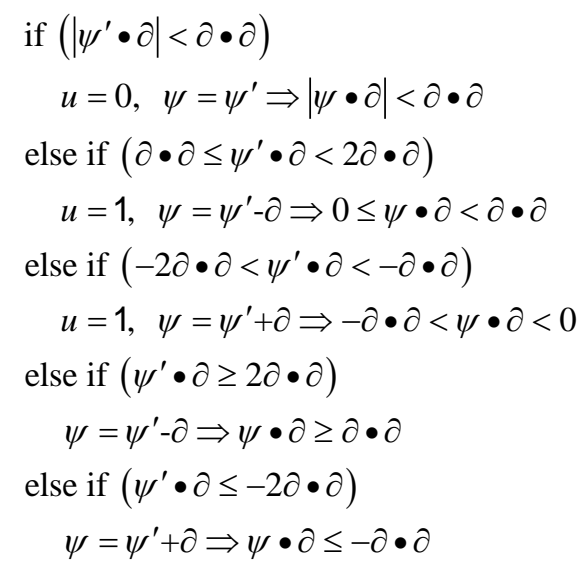

The process in (14) is the reverse process of (13). When $\left|\psi^{\prime} \bullet \partial\right|<\partial \bullet \partial$, the hidden data bit $u$ is 0 . The original carrier vector $\psi$ is not changed for hiding information 0 , i.e., $\psi=\psi^{\prime}$, so the values of QDCT coefficients do not need to be recovered. Identifying the intervals $[\partial \bullet \partial, 2 \partial \bullet \partial)$ and $(-2 \partial \bullet \partial,-\partial \bullet \partial)$ of $\psi^{\prime} \bullet \partial$, we extract one bit of information 1 . The interval $(\partial \bullet \partial, 2 \partial \bullet \partial)$ is altered to be its original interval $\left[0, \partial \bullet \partial\right.$ ) by subtracting the offset vector $\partial$ from the carrier vector $\psi^{\prime}$. The interval $(-2 \partial \bullet \partial,-\partial \bullet \partial)$ is altered to be its original interval $(-\partial \bullet \partial, 0)$ by adding the offset vector $\partial$ to the carrier vector $\psi^{\prime}$. The values of QDCT coefficients are recovered correspondingly. When the value of $\psi^{\prime} \bullet \partial$ belongs to the interval $[2 \partial \bullet \partial, \infty)$ or $(-\infty,-2 \partial \bullet \partial]$, there is no hidden message. The interval $[2 \partial \bullet \partial, \infty)$ is altered to be its original interval $[\partial \bullet \partial, \infty)$ by subtracting the offset vector $\partial$ from the carrier vector $\psi^{\prime}$. The interval $(-\infty,-2 \partial \bullet \partial]$ is altered to be its original interval $(-\infty,-\partial \bullet \partial]$ by adding the offset vector $\partial$ to the carrier vector $\psi^{\prime}$. The video is exactly restored after extracting information in this way.

In $\mathrm{P}_{0}$ frame of the right view, once $\left|Y_{00}\right| \geq H$ is satisfied by a $4 \times 4$ luminance QDCT block, but Conditions 1 to 3 are not satisfied concurrently, only Condition 1 or $\mathbf{2}$ is satisfied, pairs of coefficients from $R_{\text {set }}$ or $C_{\text {set }}$ are used to extract the information and restore the video. At last, the video is entropy encoded and the information is decrypted with keys.

Denote the frame number as $N$, and the information length as $L$. The computational efficiency of the proposed algorithm is related to the information length $L$ and the frame number $N$. Therefore, the computational complexity of the proposed algorithm can be denoted by $\mathrm{O}(N \times L)$.

Furthermore, the proposed way of limiting distortion drift could be used for 2D or 3D H.264 video with other structure. The presented lossless steganography algorithm could be used for hiding information in other media that can be grouped (some elements in a group can be selected to build a carrier vector). In addition, when the proposed method is applied in some media, especially a gray-scale image with 8 storage bits, the overflow/underflow problem should be treated[14]. However, this problem need not be considered when the data is hidden into QDCT coefficients of H.264 video.

\section{EXPERIMENTAL RESUlTS AND DisCUSSIONS}

Nine test videos (the size of each frame is 640×480) Akko \& Kayo, Ballroom, Crowd, Exit, Flamenco, Objects, Race, Rena, and Vassar [25] are utilized to do experiments with JM18.4 [26]. The parameter intra-period is 8 and two YUV files are encoded to a 3D H.264 video with 233 
frames. $30 \mathrm{P}_{0}$ frames in the right view are used to hide data. The capacity of a video sequence is the average number of bits embedded into one $\mathrm{P}_{0}$ frame of all the $\mathrm{P}_{0}$ frames in that sequence. The peak signal-to-noise ratio (PSNR) value, the structural similarity (SSIM) value, and the Kullback-Leibler divergence (KLD) value obtained through comparing the marked YUV video with the original YUV video are the averages of all the frames. The difference of PSNR (DPSNR) and the difference of SSIM (DSSIM) are discrepancies before and after hiding data. The embedding efficiency denoted as $e$ is defined by $e=L_{e m b} / L_{c h a}$, where $L_{e m b}$ is the number of embedded bits, and $L_{c h a}$ is the quantity of changed bits.

\subsection{Effect of Distortion Drift Limitation}

When the parameter code block pattern of a block is zero, there is no QDCT coefficient stored in the block which has all zero coefficients in fact. Therefore, not every block in a $640 \times 480$ frame, which has 307200 QDCT coefficients at most, could be changed, so grand visual distortion can be eliminated. The space meeting our conditions (Conditions 1 to 3, Condition 1 or 2) is not too less than unconditional space. In addition, most QDCT coefficients are zero, which can guarantee enough capacity for embedding secret information. In order to prevent intra-frame and inter-view distortion drift, the embeddable coefficients of the proposed algorithm are chosen as shown in Table 1 . Steganography with more rigorous condition will bring lesser capacity and preferable invisibility.

Denote the presented scheme without inter-view and intra-frame distortion drift as P_noDrift, the scheme without inter_view distortion drift as $\mathrm{P}_{-}$drift, and the scheme without limiting distortion drift as I_drift, where information is hidden into $\mathrm{I}_{0}$ frame. Data is hidden into $\mathrm{P}_{0}$ frame in $\mathrm{P} \_$noDrift and P_drift. The proposed lossless steganography algorithm based on orthogonal vector is used for hiding data in the three schemes, where $\partial=\partial_{c}=(0,1)$ and the threshold $\mathrm{H}=0$. The quantities of information embedded into the nine test videos are 750 bits, 1700 bits, 3800 bits, 630 bits, 880 bits, 650 bits, 2400 bits, 630 bits, and 1000 bits, respectively. As shown in Figure 5, the KLD and DPSNR values of I_drift are very large, which show that obvious distortion is caused by hiding data into I frame. So it is easy to be found by the third party, that is, its undetectability and security are weak. Through preventing inter-view distortion drift, the KLD and DPSNR value of P_drift are less than those of I_drift. Compared with I_drift, P_noDrift is superior by enhancing PSNR with $7.5 \mathrm{~dB}$ and reducing KLD with 0.07 at least. By avoiding inter-view and intra-frame distortion drift, the KLD and DPSNR value of P_noDrift are about 0 , which is hard to be detected by the third party. Therefore, the presented way to limit distortion drift is effective for improving the undetectability and security.

Table 1. Embeddable coefficients of different methods

\begin{tabular}{|c|c|c|c|}
\hline Methods & Conditions 1 to 3 & Condition 1 $\left(\boldsymbol{R}_{\text {set }}\right)$ & Condition 2 $\left(\boldsymbol{C}_{\text {set }}\right)$ \\
\hline Ours & $\psi=\left(Y_{11}, Y_{22}\right)$ & $\begin{array}{c}\psi_{c}=\left(Y_{01}, Y_{02}\right) \\
\omega=\left(Y_{21}, Y_{22}\right)\end{array}$ & $\begin{array}{c}\psi_{c}=\left(Y_{10}, Y_{20}\right) \\
\omega=\left(Y_{12}, Y_{22}\right)\end{array}$ \\
\hline HS & $Y_{22}$ & $\left(Y_{02}, Y_{22}\right)$ & $\left(Y_{20}, Y_{22}\right)$ \\
\hline DE & $\left(Y_{11}, Y_{22}\right)$ & $\left(Y_{02}, Y_{22}\right)$ & $\left(Y_{20}, Y_{22}\right)$ \\
\hline IPS & $\left(Y_{11}, Y_{22}\right)$ & $\left(Y_{02}, Y_{22}\right)$ & $\left(Y_{20}, Y_{22}\right)$ \\
\hline
\end{tabular}




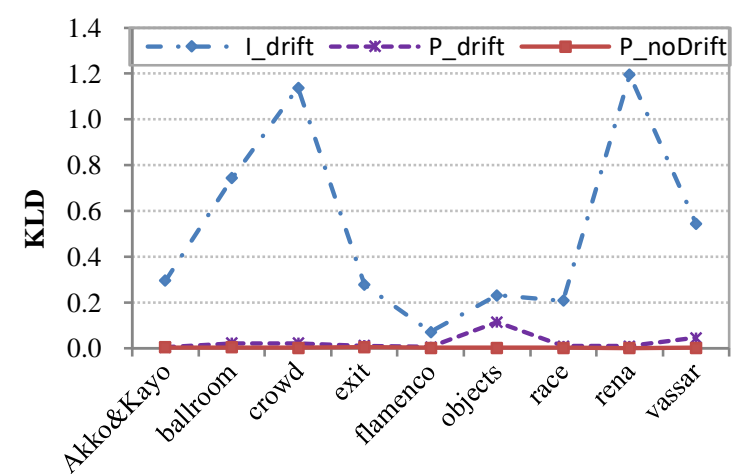

3D H.264 video sequences

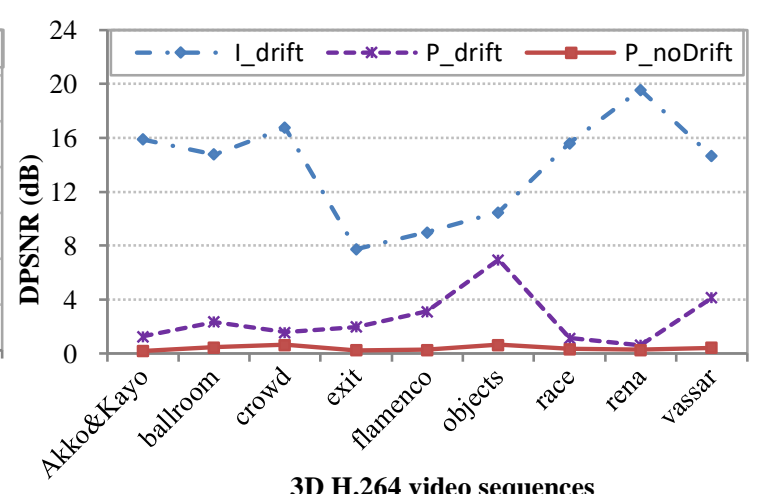

3D H.264 video sequences

(a) (b)

Figure 5. KLD and PSNR of the schemes with or without distortion drift limitation

Correspondingly, the marked frames of Flamenco and Race are shown in Figure 6 (a) and (e) are the first original $\mathrm{P}_{0}$ frames, (b) and (f) are the first marked $\mathrm{I}_{0}$ frames obtained by using I_drift to hide data, (c) and $(\mathrm{g})$ are the first marked $\mathrm{P}_{0}$ frames obtained by using $\mathrm{P}_{-}$drift to hide data, $(\mathrm{d})$ and (h) are the first marked $\mathrm{P}_{0}$ frames obtained by using $\mathrm{P}_{-}$noDrift to hide data. It can be seen that there are large distortion in the frames (b) and (f). The distortion is around the people and floor in the frame (b). The road and trees are distorted in the frame (f). When data is hidden into the first $\mathrm{P}_{0}$ frame, there is no distortion on the first $I_{0}$ frame. Compared with the frames (b) and (f), the distortion in the frames (c) and (g) is less. Furthermore, the distortion in the frames (d) and (h) is not obvious. It can be concluded from the results that superior visual quality and invisibility could be achieved by using the proposed way to prevent inter-view and intra-frame distortion drift.

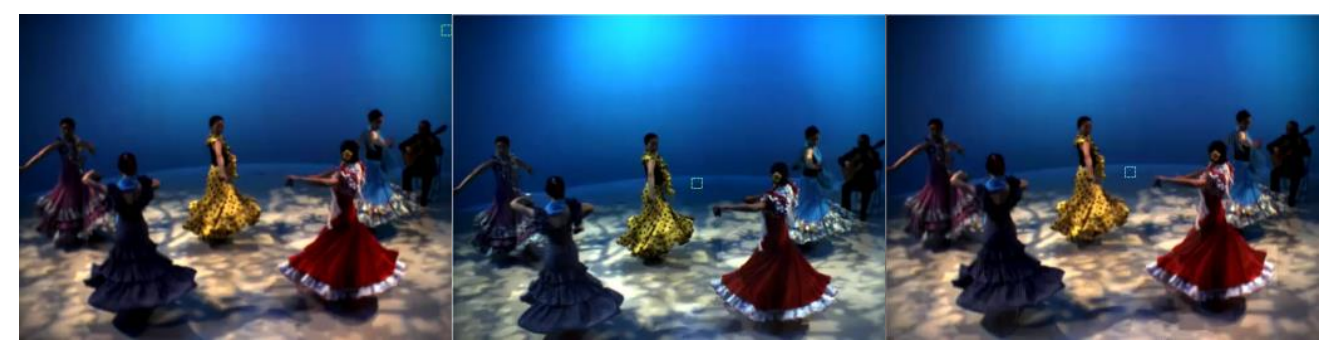

(a)

(b)

(c)

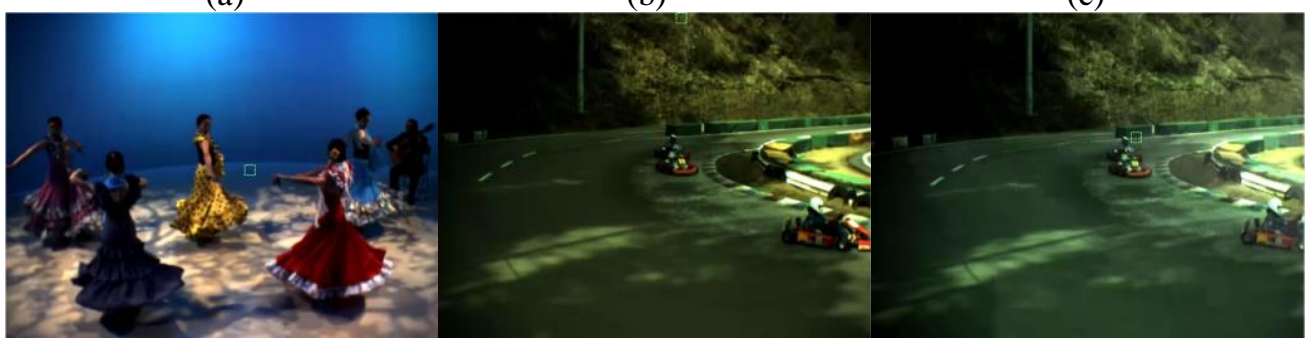

(d)

(e)

(f)

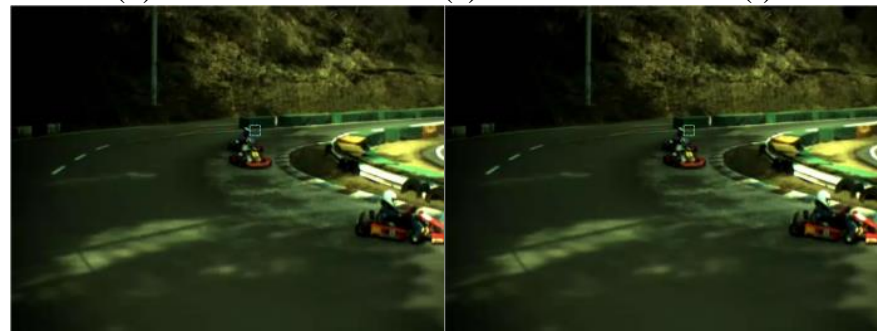

(g)

(h)

Figure 6. The original and marked frames of Flamenco and Race 


\subsection{Comparison of Different Lossless Steganography Methods}

In order to compare the presented algorithm with other lossless steganography methods in the same environment, embeddable blocks, which could be used for hiding information without causing inter-view and intra-frame distortion drift, are selected from $\mathrm{P}_{0}$ frames by using three conditions and coupling coefficients. HS[8], DE[15], and IPS [16] are three typical lossless steganography algorithms and could be used for video. Therefore, they are chosen for comparing with our algorithm. The embeddable coefficients of the proposed algorithm, HS [8], DE [15], and IPS [16] are shown in Table 1. Denote the proposed scheme with the offset vector $\partial=\partial_{c}=(0,1)$ as $\operatorname{Our}(0.1)$, and the proposed scheme with the offset vector $\partial=\partial_{c}=(0,2)$ as $\operatorname{Our}(0,2)$.The comparison of embedding performance for different schemes is portrayed in Figure 7, where the points of each line from left to right represent the embedding cases in which the threshold $H$ is $4,3,2,1$, and 0 , respectively.

Compared with other schemes, in order to embed the same quantity of information, the least DSSIM and DPSNR (i.e. the best SSIM and PSNR) can be obtained by using the proposed algorithm Our (0.1). The best SSIM and PSNR mean that the best video quality, invisibility and undetectability. Let HSO be HS that is employed for embedding information into zero coefficients, and $\mathrm{HS} \pm 1$ be $\mathrm{HS}$ that is employed for embedding information into 1 or -1 coefficients. Given the same conditions and coupling coefficients to prevent intra-frame distortion propagation, HSO is equivalent to the presented algorithm Our $(0,1)$. So the lines of HS0 are thusly omitted in Figure 7. Little capacity, embedding efficiency, DSSIM and DPSNR could be got by using HS \pm 1 to hide data.

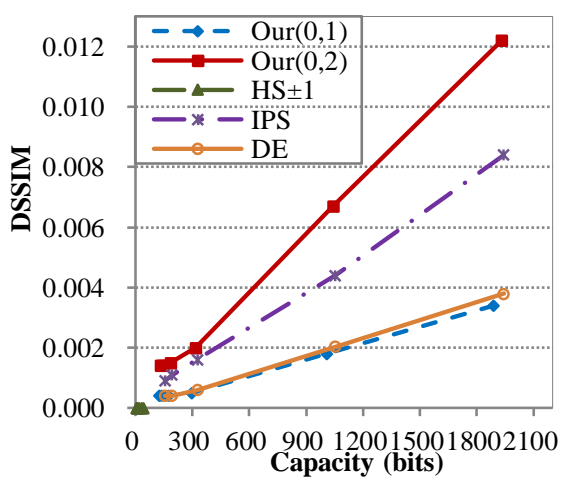

(a) Ballroom

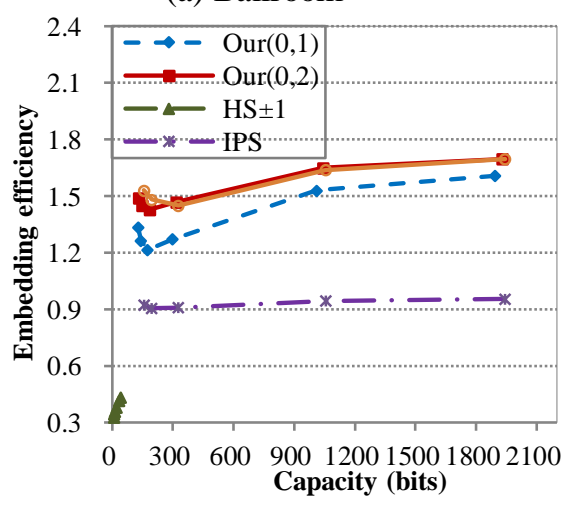

(c) Ballroom

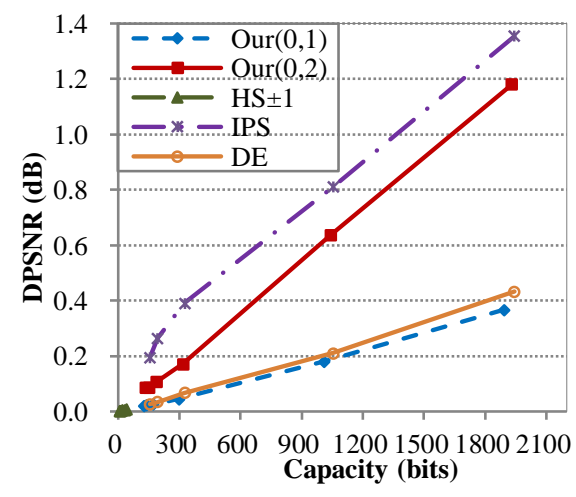

(b) Ballroom

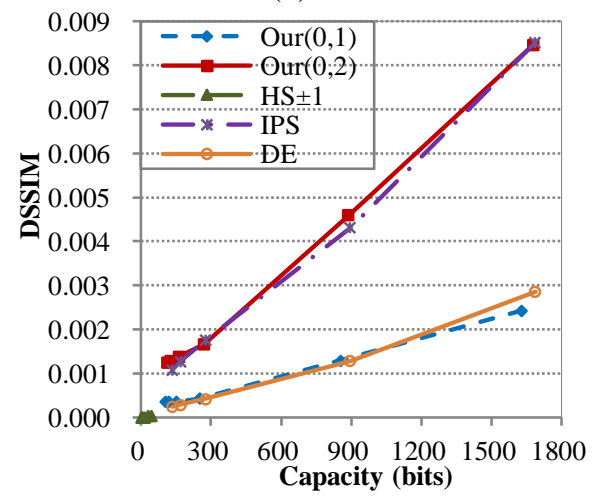

(d) Average of 9 videos 


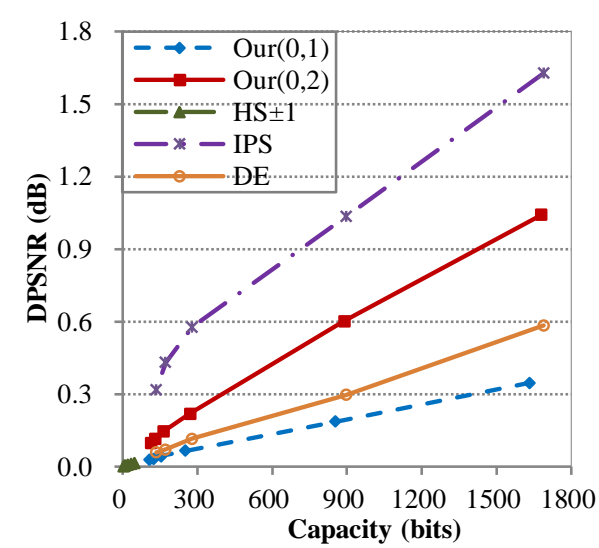

(e) Average of 9 videos

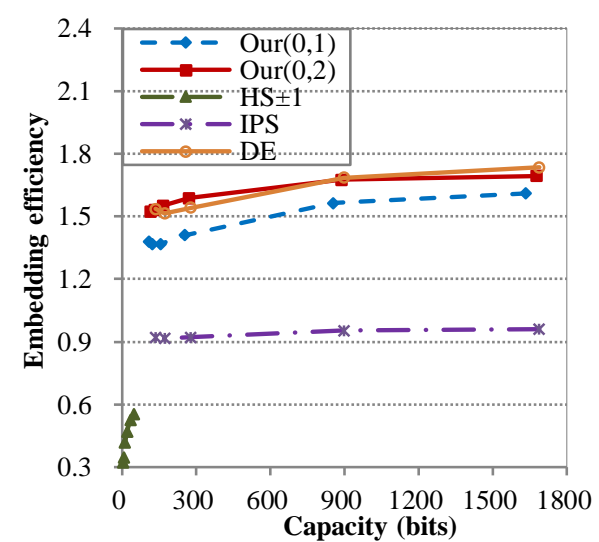

(f) Average of 9 videos

Figure 7. Performance comparison of varied lossless algorithms

Compared with Our( 0,1$)$, higher capacity and better embedding efficiency can be achieved by using $\operatorname{Our}(0,2)$ to hide information. At the same time, the higher DSSIM and DPSNR are caused. That is, when schemes with bigger $|\partial|$ or $\left|\partial_{c}\right|$ are used to hide data, greater capacity can be got, and more distortion will be begot. Therefore, we need to control the modulus of $\partial$ for limiting the distortion.

\subsection{Discussions}

When the threshold $\mathrm{H}$ is not considered, the optional number for picking $n$ coefficients from 16 QDCT coefficients is the number of permutations, which is denoted by $A_{16}^{n}$. In an embeddable block satisfying Conditions 1 to 3 at the same time, $A_{16}^{n}$ ways could be used to create the carrier vector $\psi$. Assume the element $z_{i}$ in the offset vector $\partial$ is 0,1 or -1 , there are $3^{n}-1$ kinds of $\partial$ excluding a zero vector. Then the amount of selections for constructing $\psi$ and $\partial$ is calculated by (15). Even if an eavesdropper knows a marked block, the probability of guessing the embedded value directly is only $1 /\left(1.3 \times 10^{21}\right) \approx 7.7 \times 10^{-22}$.

$$
\sum_{n=2}^{16} A_{16}^{n}\left(3^{n}-1\right)=\sum_{n=2}^{16} \frac{16 !\left(3^{n}-1\right)}{(16-n) !} \approx 1.3 \times 10^{21} .
$$

When only one $z_{i}$ in the offset vector $\partial$ is 1 or -1 and the others are 0 , there are $n$ ways to choose one $z_{i}$ from $n$ elements of $\partial$. This $z_{i}$ could be 1 or $-1,2$ ways could be used. So $2 n$ alterations could be used to form $\partial$, and the optional count for constructing $\psi$ and $\partial$ is obtained by (16). Even if the third party identifies a marked block and the data hiding algorithm, the probability for calculating the hidden data bit at once is only $1 /\left(1.7 \times 10^{15}\right) \approx 5.9 \times 10^{-16}$.

$$
2 \sum_{n=2}^{16} n A_{16}^{n}=2 \sum_{n=2}^{16} \frac{16 ! n}{(16-n) !} \approx 1.7 \times 10^{15} .
$$

When only one $z_{i}$ in the offset vector $\partial_{c}$ is 1 or -1 and the others are $0,2 S$ alterations could be used to form $\partial_{c}$, and the optional count for constructing $\psi_{c}$ and $\partial_{c}$ is

$$
2 \sum_{S=2}^{8} S 2^{S} A_{8}^{S}=2 \sum_{S=2}^{8} \frac{8 ! S 2^{S}}{(8-S) !} \approx 2.6 \times 10^{8} .
$$


Given a pure payload, only 16 selections could be used by HS for picking one position from 16 QDCT coefficients to embed data and the elective sum for DE and IPS is $A_{16}^{2}=240$. It can be seen clearly that our lossless algorithm has an enormous choice, which can be united with large capacity to provide a broad operational space for secure and robust technologies someday. The proposed algorithm will be combined with random function, secret sharing, duplication code, error correcting code, and other methods to strengthen the security and robustness of covert communication in future.

\section{CONCLUSiOnS}

A lossless steganography algorithm based on orthogonal vector with limited distortion propagation for 3D H.264 video is proposed to acquire fine embedding performance. Inter-view and intra-frame distortion diffusion is prevented according to the prediction structure and modes of 3D video. Some conditions are used to select an embeddable block, where several coefficients are picked up to compose a carrier vector or compensation vector. One bit of message is embedded or extracted according to the value of the inner product of the carrier vector and offset vector. There is such a huge choice space to create and change a carrier vector that a large operational area is achieved for steganography. Compared with other algorithms, the proposed scheme has superior invisibility and undetectability because of the numerous ways of forming carrier vector and offset vector.

\section{ACKNOWLEDGMENT}

This work is supported by the Natural Science Foundation of Hubei Prorince under Grant 2017CFB306 .

The authors are heartily grateful to the reviewers for their valuable comments improving the quality of the original manuscript.

\section{REFERENCES}

[1] X. P. Zhang, "Reversible data hiding with optimal value transfer," (in English), IEEE Transactions on Multimedia, Article vol. 15, no. 2, pp. 316-325, Feb 2013.

[2] C. Y. Yang, L. T. Cheng, and W. F. Wang, "An efficient reversible ECG steganography by adaptive LSB approach based on 1D FDCT domain," Multimedia Tools and Applications, vol. 79, no. 33-34, pp. 24449-24462, Sep 2020.

[3] Z. C. Ni, Y. Q. Shi, N. Ansari, and W. Su, "Reversible data hiding," (in English), IEEE Transactions on Circuits and Systems for Video Technology, Article vol. 16, no. 3, pp. 354-362, Mar 2006.

[4] X. T. Wang, C. C. Chang, T. S. Nguyen, and M. C. Li, "Reversible data hiding for high quality images exploiting interpolation and direction order mechanism," (in English), Digital Signal Processing, Article vol. 23, no. 2, pp. 569-577, Mar 2013.

[5] Y. Y. Tsai, D. S. Tsai, and C. L. Liu, "Reversible data hiding scheme based on neighboring pixel differences," Digital Signal Processing, vol. 23, no. 3, pp. 919-927, May 2013.

[6] W. Hong, T. S. Chen, and M. C. Wu, "An improved human visual system based reversible data hiding method using adaptive histogram modification," Optics Communications, vol. 291, pp. 87-97, Mar 2013.

[7] J. Zhao and Z. Li, "Three-dimensional histogram shifting for reversible data hiding," Multimedia Systems, vol. 24, no. 1, pp. 95-109, February 19,2018 2018.

[8] X. Z. Xie, C. C. Chang, and Y. C. Hu, "An adaptive reversible data hiding scheme based on prediction error histogram shifting by exploiting signed-digit representation," Multimedia Tools and Applications, vol. 79, no. 33-34, pp. 24329-24346, Sep 2020.

[9] W. F. Qi, X. L. Li, T. Zhang, and Z. M. Guo, "Optimal Reversible Data Hiding Scheme Based on Multiple Histograms Modification," Ieee Transactions on Circuits and Systems for Video Technology, vol. 30, no. 8, pp. 2300-2312, Aug 2020. 
[10] J. Zhao, Z. Li, and B. Feng, "A novel two-dimensional histogram modification for reversible data embedding into stereo H.264 video," Multimedia Tools and Applications, vol. 75, no. 10, pp. 5959-5980, May 1, 20162016.

[11] J. Tian, "Reversible data embedding using a difference expansion," (in English), IEEE Transactions on Circuits and Systems for Video Technology, Article vol. 13, no. 8, pp. 890-896, Aug 2003.

[12] O. M. Al-Qershi and B. E. Khoo, "Two-dimensional difference expansion (2D-DE) scheme with a characteristics-based threshold," Signal Processing, vol. 93, no. 1, pp. 154-162, Jan 2013.

[13] X. L. Li, J. Li, B. Li, and B. Yang, "High-fidelity reversible data hiding scheme based on pixel-value-ordering and prediction-error expansion," Signal Processing, vol. 93, no. 1, pp. 198-205, Jan 2013.

[14] W. Q. Wang, "A reversible data hiding algorithm based on bidirectional difference expansion," Multimedia Tools and Applications, vol. 79, no. 9-10, pp. 5965-5988, Mar 2020.

[15] C. Y. Weng, "DWT-based reversible information hiding scheme using prediction-error-expansion in multimedia images," Peer-to-Peer Networking and Applications, vol. 13, no. 2, pp. 514-523, Mar 2020.

[16] S. Maiti and M. P. Singh, "A novel reversible data embedding method for source authentication and tamper detection of H.264/AVC video," presented at the 5th International Conference on Information Processing, ICIP 2011, Bangalore, India, August 5, 2011 - August 7, 2011, 2011. Available: <Go to ISI>://WOS:000306579700044

[17] T. Zhang, X. L. Li, W. F. Qi, and Z. M. Guo, "Location-Based PVO and Adaptive Pairwise Modification for Efficient Reversible Data Hiding," Ieee Transactions on Information Forensics and Security, vol. 15, pp. 2306-2319, 2020.

[18] B. G. Mobasseri, R. J. Berger, M. P. Marcinak, and Y. J. NaikRaikar, "Data Embedding in JPEG Bitstream by Code Mapping," Ieee Transactions on Image Processing, vol. 19, no. 4, pp. 958-966, Apr 2010.

[19] X. T. Wu, C. N. Yang, and Y. W. Liu, "A general framework for partial reversible data hiding using hamming code," Signal Processing, vol. 175, Oct 2020, Art. no. 107657.

[20] Y. Liu, S. Liu, Y. Wang, H. Zhao, and S. Liu, "Video steganography: A review," Neurocomputing, vol. 335, pp. 238-250, 2019/03/28/ 2019.

[21] X. J. Ma, Z. T. Li, H. Tu, and B. C. Zhang, "A data hiding algorithm for H.264/AVC video streams without intra-frame distortion drift," (in English), IEEE Transactions on Circuits and Systems for Video Technology, vol. 20, no. 10, pp. 1320-1330, Oct 2010.

[22] J. Franco-Contreras, S. Baudry, and G. Doërr, "Virtual view invariant domain for 3D video blind watermarking," in Image Processing (ICIP), 2011 18th IEEE International Conference on, 2011, pp. 2761-2764: IEEE.

[23] A. Koz, C. Cigla, and A. A. Alatan, "Watermarking of free-view video," Image Processing, IEEE Transactions on, vol. 19, no. 7, pp. 1785-1797, 2010.

[24] A. Chammem, M. Mitrea, and F. Preteux, "Stereoscopic video watermarking: a comparative study," Annals of Telecommunications-Annales Des Telecommunications, vol. 68, no. 11-12, pp. 673-690, Dec 2013.

[25] (Feb). Video test sequences. Available: http://blog.csdn.net/do2jiang/article/details/5499464

[26] K. Sühring. (Aug). H.264/AVC software coordination (JM 18.4 ed.). Available: http://iphome.hhi.de/suehring/tml 


\section{AUTHORS}

Juan Zhao received her B.S. degree from Henan Normal University, Xinxiang, China, in 2007, and PhD degree from Huazhong University of Science and Technology, Wuhan, China, in 2015. She is currently a lecturer in School of Mathematics \&Computer Science, Wuhan Polytechnic University. Her research interests include data hiding, network security and multimedia security.

Zhitang Li received his M.E. degree in Computer Architecture from Huazhong University of Science and Technology, Wuhan, China, 1987, and PhD degree in Computer Architecture from Huazhong University of Science and Technology, Wuhan, China, 1992. His research interests include computer architecture, network security, and P2P networks. He was the director of China Education and Research Network in Central China. He was a vice president of Department of Computer Science and Technology, Huazhong University of Science and Technology, China. He has published more than one hundred papers in the areas of network security, computer architecture, and P2P networks.

(C) 2021 By AIRCC Publishing Corporation. This article is published under the Creative Commons Attribution (CC BY) license. 


\title{
REVERSIBLE DATA HIDING BASED ON TWO-DIMENSIONAL HISTOGRAM SHIFTING
}

\author{
Juan Zhao ${ }^{1}$ and Zhitang $\mathrm{Li}^{2}$ \\ ${ }^{1}$ School of Mathematics \&Computer Science, Wuhan Polytechnic University, \\ Wuhan 430048, China \\ ${ }^{2}$ Institute of Computer Science and Technology, Huazhong University of \\ Science and Technology, Wuhan 430074, China
}

\begin{abstract}
This paper presents a two-dimensional histogram shifting technique for reversible data hiding algorithm. In order to avoid the distortion drift caused by hiding data into stereo H.264 video, we choose arbitrary embeddable blocks from $4 \times 4$ quantized discrete cosine transform luminance blocks which will not affect their adjacent blocks. Two coefficients in each embeddable block are chosen as a hiding coefficient pair. The selected coefficient pairs are classified into different sets on the basis of their values. Data could be hidden according to the set which the value of the coefficient pair belongs to. When the value of one coefficient may be changed by adding or subtracting 1, two data bits could be hidden by using the proposed method, whereas only one data bit could be embedded by employing the conventional histogram shifting. Experiments show that this two-dimensional histogram shifting method can be used to improve the hiding performance.
\end{abstract}

\section{KEYWORDS}

Reversible data hiding, Two-dimensional histogram shifting, H.264, Multi-view coding

\section{INTRODUCTION}

Using data hiding including watermarking and steganography, we embed data into multimedia for covert communication, authentication, annotation, fingerprinting and copyright protection. In some applications such as medical media sharing, military, law enforcement, multimedia file management and remote sensing, the permanent media distortion caused by data hiding is intolerable. Reversible data hiding $(\mathrm{RDH})$ can fully restore the host media after extracting data. Hiding data into the quantized discrete cosine transformation (QDCT) coefficients is the most common practice, but the distortion due to embedded data will spread and accumulate, called distortion drift. By a video RDH algorithm, the value of each QDCT coefficient is restored completely, so important videos without distortion could be treasured and watched. Consequently, there will not be too many network videos with hidden important information so that it is difficult for others to find stego covers. Additionally, some video RDH techniques have been presented for sensitive applications [1] and error concealment [2, 3] recently.

In order to improve the hiding capacity and reduce the distortion, algorithms based on histogram shifting (HS) [2-14], difference expansion [15-18], integer transform [1, 19, 20] and soon have been proposed recently, where HS, difference expansion, and integer transform are three main techniques that could be used for hiding data into H.264 videos. 
The peak of the image histogram is used to hide data in the typical HS scheme [6], where each pixel value is changed at most by adding or subtracting 1 . When the peak point value is higher, the higher capacity and lower distortion could be obtained. So the difference and the predictionerror HS methods are presented later. Difference image histogram shifting is utilized for multilevel RDH in [8], where a value in a difference image is the difference between two neighboring pixels in an image. Nine-dimensional histogram is applied for prediction strategy in [10], where prediction-error expansion techniques and pixel selection methods are combined with the general framework of HS-based RDH to achieve better hiding performance. In [12], a bidirectional-shift strategy is used to extend the shift able positions in the central zone of the allowable coordinates. QDCT coefficient histogram is similar to difference histogram and prediction-error histogram. So it is an efficient and welcome way to reversibly hide data into QDCT coefficients by using HS in H.264 video RDH schemes [2, 3]. However, in the conventional HS scheme, each coefficient, difference, or prediction error is changed in isolation.

With the rapid development of popular stereo video, traditional RDH technique researchers began to turn their attention to the new cover media. Joint Video Team released multi-view video coding (MVC) standard as an appendix of H.264 standard in 2010. In order to compress efficiently, intra-frame prediction, inter-frame prediction and parallax prediction are used by MVC standard. Hence, embedding information into MVC video may cause visual distortion and serious distortion drift. Therefore, a two-dimensional (2D) HS-based RDH algorithm for MVC video with limited distortion drift is proposed in this paper. Compared with other methods, enhanced 2D HS technique selecting two arbitrary QDCT AC coefficients from every embeddable block for embedding more information with lower distortion.

The rest of the paper is organized as follows. In section 2, the main idea of 2D HS is introduced. Section 3 describes the way of preventing distortion drift for MVC video and depicts the MVC video RDH algorithm based on 2D HS. Experimental results are given in section 4. Last, conclusions are drawn in section 5 .

\section{HS-BASED RDH SCHEME}

\subsection{Conventional HS}

One-dimensional (1D) HS proposed by Ni et al. [6] could be used for hiding data into QDCT coefficients of MVC video. The peak of QDCT coefficient histogram is 0. Information is embedded by expansion and shifting as shown in Figure 1 and (1).

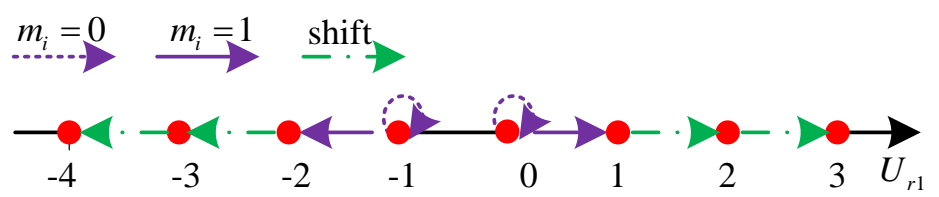

Figure 1. Transformation of QDCT coefficients in conventional 1D HS

$$
U_{r 1}^{\prime}= \begin{cases}U_{r 1}, & \text { if }\left(U_{r 1}=0 \text { or }-1\right) \wedge\left(m_{i}=0\right) \\ 1, & \text { if }\left(U_{r 1}=0\right) \wedge\left(m_{i}=1\right) \\ -2, & \text { if }\left(U_{r 1}=-1\right) \wedge\left(m_{i}=1\right) \\ U_{r 1}+1, & \text { if } U_{r 1}>0 \\ U_{r 1}-1, & \text { if } U_{r 1}<-1\end{cases}
$$


Where $U_{r 1}$ is a QDCT coefficient, $U_{r 1}^{\prime}$ is the marked QDCT coefficient after hiding data, and $m_{i}$ $\in\{0,1\}$ is a to-be-embedded data bit. When the value of $U_{r 1}$ is 0 or -1 , a data bit could be hidden, where the video will not be modified if $m_{i}$ is " 0 ", and the coefficient will become 1 or -2 if $m_{i}$ is "1". Accordingly, the receiver could extract information $m_{i}$ and restore the original QDCT coefficient from the marked QDCT coefficient ${ }_{r 1}^{\prime}$ as follows:

A. If the value of the marked coefficient $U_{r 1}^{\prime}$ is 0 or -1 , the extracted data bit $m_{i}$ will be 0 , the original coefficient $U_{r 1}={ }_{r 1}^{\prime}$, which needs no modification.

B. If $U_{r 1}^{\prime}$ is 1 , the original value of the coefficient $U_{r 1}$ will be 0 , and the bit $m_{i}$ will be 1 .

C. If $U_{r 1}^{\prime}$ is -2 , the original value of the coefficient $U_{r 1}$ will be -1 , and the bit $m_{i}$ will be 1 .

D. If $U_{r 1}^{\prime}>1$, there will be no hidden data bit, and the original coefficient $U_{r 1}=U_{r 1}^{\prime}-1$.

E. If $U_{r 1}^{\prime}<-2$, there will be no hidden data bit, and the original coefficient $U_{r 1}={ }_{r 1}^{\prime}+1$.

In this scheme, the $1 \mathrm{D}$ coefficient histogram is defined by

$$
h\left(d_{1}\right)=\#\left\{U_{r 1} \mid U_{r 1}=d_{1}\right\}
$$

Where \# is the cardinal number of a set, and $d_{1}$ is an integer.

The embedding capacity denoted as EC of the 1D HS hiding scheme is

$$
E C=h(0)+h(-1)
$$

For QDCT coefficients, the embedding distortion denoted as ED in terms of $l^{2}$-error can be formulated as

$$
E D=\frac{1}{2} h(0)+\frac{1}{2} h(-1)+\sum_{d_{1}=-\infty}^{-2} h\left(\mathrm{~d}_{1}\right)+\sum_{d_{1}=1}^{+\infty} h\left(\mathrm{~d}_{1}\right)
$$

When two QDCT coefficients denoted as $U_{r 1}$ and $U_{r 2}$ are used to embed data, the mapping shown in Figure 1 can be used for hiding data into each coefficient. Therefore, a 2D coefficient histogram defined by (5) could be employed to hide data, as shown in Figure 2, where at most two bits of information denoted by $m_{i} m_{i+1}$ could be hidden into one pair of coefficients.

$$
t\left(d_{1}, d_{2}\right)=\#\left\{\left(U_{r 1}, U_{r 2}\right) \mid U_{r 1}=d_{1}, U_{r 2}=d_{2}\right\}
$$

where $d_{2}$ is an integer.

\subsection{Proposed 2D HS}

In Figure 2, the maximum modification of each QDCT coefficient pair is 2, which may cause obvious distortion. In order to reduce the cost, we primarily seek for different points to record different data with at most one modification. Four points are needed for recording two bits of data "00", "01", "10", and "11". As illustrated in Figure 3, when the value of coefficient pair $\left(U_{r 1}\right.$, $\left.U_{r 2}\right)$ is $(-1,0)$, which can be used for representing " 00 " with no change, it could be expanded to its neighboring points $(-1,1),(-2,0)$, and $(-1,-1)$ to record two data bits 01,10 , and 11 with one modification, respectively. When the value of $\left(U_{r 1}, U_{r 2}\right)$ is $(2,0)$, it can be expanded to its adjacent points $(3,0)$ and $(2,1)$ for recording a data bit " 0 " and " 1 " with one modification, 
respectively. According to these mapping rules, the set (denoted as $D$ ) of all the values of QDCT coefficient pairs, could be divided into sixteen subsets defined as follows:

$D_{1}=\{(0,0)\}, \quad D_{2}=\{(-1,0)\}, D_{3}=\{(-1,-1)\}, D_{4}=\{(0,-1)\}, \quad D_{5}=\left\{\left(U_{r 1}, 0\right) \mid U_{r 1}>0\right\}$,

$D_{6}=\left\{\left(U_{r 1}, 0\right) \mid U_{r 1}<-1\right\}, D_{7}=\left\{\left(U_{r 1},-1\right) \mid U_{r 1}>0\right\}, D_{8}=\left\{\left(U_{r 1},-1\right) \mid U_{r 1}<-1\right\}$,

$D_{9}=\left\{\left(0, U_{r 2}\right) \mid U_{r 2}>0\right\}, D_{10}=\left\{\left(0, U_{r 2}\right) \mid U_{r 2}<-1\right\}, D_{11}=\left\{\left(-1, U_{r 2}\right) \mid U_{r 2}>0\right\}$,

$D_{12}=\left\{\left(-1, U_{r 2}\right) \mid U_{r 2}<-1\right\}, D_{13}=\left\{\left(U_{r 1}, U_{r 2}\right) \mid U_{r 1}>0, U_{r 2}>0\right\}$,

$D_{14}=\left\{\left(U_{r 1}, U_{r 2}\right) \mid U_{r 1}>0, U_{r 2}<-1\right\}, D_{15}=\left\{\left(U_{r 1}, U_{r 2}\right) \mid U_{r 1}<-1, U_{r 2}>0\right\}$,

$D_{16}=\left\{\left(U_{r 1}, U_{r 2}\right) \mid U_{r 1}<-1, U_{r 2}<-1\right\}$.

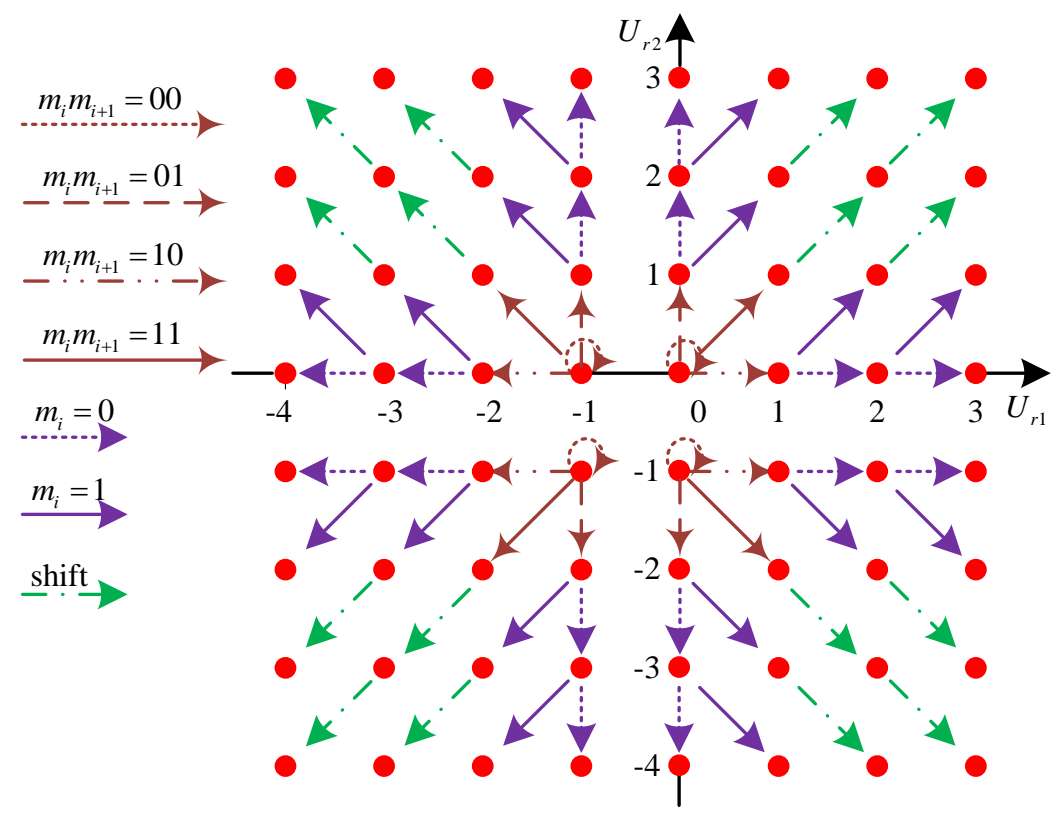

Figure 2. Transformation of QDCT coefficients in conventional 2D HS

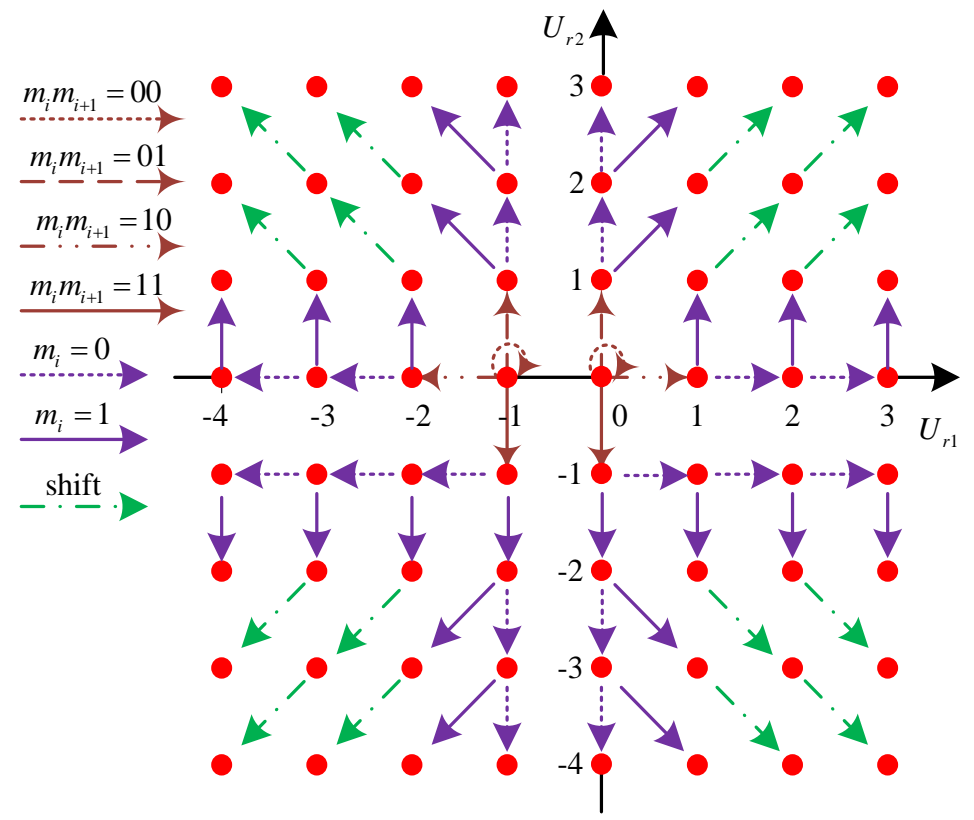

Figure 3. Transformation of QDCT coefficients in the proposed 2D HS 
The selected coefficient pairs are classified into different sets on the basis of their values. Information could be hidden according to the set which the value of the coefficient pair belongs to. Correspondingly, the embedding process can be described in the following way.

If the coefficient pair $\left(U_{r 1}, U_{r 2}\right) \in D_{1}$, the marked coefficient pair denoted by $\left(U_{r 1}^{\prime}, U_{r 2}^{\prime}\right)$ will be

$$
\left(U_{r 1}^{\prime}, U_{r 2}^{\prime}\right)= \begin{cases}\left(U_{r 1}, U_{r 2}\right), & \text { if } m_{i} m_{i+1}=00 \\ \left(U_{r 1}, U_{r 2}+1\right), & \text { if } m_{i} m_{i+1}=01 \\ \left(U_{r 1}+1, U_{r 2}\right), & \text { if } m_{i} m_{i+1}=10 \\ \left(U_{r 1}, U_{r 2}-1\right), & \text { if } m_{i} m_{i+1}=11\end{cases}
$$

If the coefficient pair $\left(U_{r 1}, U_{r 2}\right) \in D_{2}$, the marked coefficient pair $\left(U_{r 1}^{\prime}, U_{r 2}^{\prime}\right)$ will be

$$
\left(U_{r 1}^{\prime}, U_{r 2}^{\prime}\right)= \begin{cases}\left(U_{r 1}, U_{r 2}\right), & \text { if } m_{i} m_{i+1}=00 \\ \left(U_{r 1}, U_{r 2}+1\right), & \text { if } m_{i} m_{i+1}=01 \\ \left(U_{r 1}-1, U_{r 2}\right), & \text { if } m_{i} m_{i+1}=10 \\ \left(U_{r 1}, U_{r 2}-1\right), & \text { if } m_{i} m_{i+1}=11\end{cases}
$$

If the coefficient pair $\left(U_{r 1}, U_{r 2}\right)$ belongs to $D_{3}$ or $D_{8}$, the marked coefficient pair $\left(U_{r 1}^{\prime}, U_{r 2}^{\prime}\right)$ will be

$$
\left(U_{r 1}^{\prime}, U_{r 2}^{\prime}\right)= \begin{cases}\left(U_{r 1}-1, U_{r 2}\right), & \text { if } m_{i}=0 \\ \left(U_{r 1}, U_{r 2}-1\right), & \text { if } m_{i}=1\end{cases}
$$

If the coefficient pair $\left(U_{r 1}, U_{r 2}\right)$ belongs to $D_{4}$ or $D_{7}$, the marked coefficient pair $\left(U_{r 1}^{\prime}, U_{r 2}^{\prime}\right)$ will be

$$
\left(U_{r 1}^{\prime}, U_{r 2}^{\prime}\right)= \begin{cases}\left(U_{r 1}+1, U_{r 2}\right), & \text { if } m_{i}=0 \\ \left(U_{r 1}, U_{r 2}-1\right), & \text { if } m_{i}=1\end{cases}
$$

If the coefficient pair $\left(U_{r 1}, U_{r 2}\right) \in D_{5}$, the marked coefficient pair $\left(U_{r 1}^{\prime}, U_{r 2}^{\prime}\right)$ will be

$$
\left(U_{r 1}^{\prime}, U_{r 2}^{\prime}\right)= \begin{cases}\left(U_{r 1}+1, U_{r 2}\right), & \text { if } m_{i}=0 \\ \left(U_{r 1}, U_{r 2}+1\right), & \text { if } m_{i}=1\end{cases}
$$

If the coefficient pair $\left(U_{r 1}, U_{r 2}\right) \in D_{6}$, the marked coefficient pair $\left(U_{r 1}^{\prime}, U_{r 2}^{\prime}\right)$ will be

$$
\left(U_{r 1}^{\prime}, U_{r 2}^{\prime}\right)= \begin{cases}\left(U_{r 1}-1, U_{r 2}\right), & \text { if } m_{i}=0 \\ \left(U_{r 1}, U_{r 2}+1\right), & \text { if } m_{i}=1\end{cases}
$$

If the coefficient pair $\left(U_{r 1}, U_{r 2}\right) \in D_{9}$, the marked coefficient pair $\left(U_{r 1}^{\prime}, U_{r 2}^{\prime}\right)$ will be

$$
\left(U_{r 1}^{\prime}, U_{r 2}^{\prime}\right)= \begin{cases}\left(U_{r 1}, U_{r 2}+1\right), & \text { if } m_{i}=0 \\ \left(U_{r 1}+1, U_{r 2}+1\right), & \text { if } m_{i}=1\end{cases}
$$


If the coefficient pair $\left(U_{r 1}, U_{r 2}\right) \in D_{10}$, the marked coefficient pair $\left(U_{r 1}^{\prime}, U_{r 2}^{\prime}\right)$ will be

$$
\left(U_{r 1}^{\prime}, U_{r 2}^{\prime}\right)= \begin{cases}\left(U_{r 1}, U_{r 2}-1\right), & \text { if } m_{i}=0 \\ \left(U_{r 1}+1, U_{r 2}-1\right), & \text { if } m_{i}=1\end{cases}
$$

If the coefficient pair $\left(U_{r 1}, U_{r 2}\right) \in D_{11}$, the marked coefficient pair $\left(U_{r 1}^{\prime}, U_{r 2}^{\prime}\right)$ will be

$$
\left(U_{r 1}^{\prime}, U_{r 2}^{\prime}\right)= \begin{cases}\left(U_{r 1}, U_{r 2}+1\right), & \text { if } m_{i}=0 \\ \left(U_{r 1}-1, U_{r 2}+1\right), & \text { if } m_{i}=1\end{cases}
$$

If the coefficient pair $\left(U_{r 1}, U_{r 2}\right) \in D_{12}$, the marked coefficient pair $\left(U_{r 1}^{\prime}, U_{r 2}^{\prime}\right)$ will be

$$
\left(U_{r 1}^{\prime}, U_{r 2}^{\prime}\right)= \begin{cases}\left(U_{r 1}, U_{r 2}-1\right), & \text { if } m_{i}=0 \\ \left(U_{r 1}-1, U_{r 2}-1\right), & \text { if } m_{i}=1\end{cases}
$$

If the coefficient pair $\left(U_{r 1}, U_{r 2}\right)$ belongs to $D_{13}, D_{14}, D_{15}$, or $D_{16}$, no message could be hidden, and the marked coefficient pair will be

$$
\left(U_{r 1}^{\prime}, U_{r 2}^{\prime}\right)= \begin{cases}\left(U_{r 1}+1, U_{r 2}+1\right), & \text { if }\left(U_{r 1}, U_{r 2}\right) \in D_{13} \\ \left(U_{r 1}+1, U_{r 2}-1\right), & \text { if }\left(U_{r 1}, U_{r 2}\right) \in D_{14} \\ \left(U_{r 1}-1, U_{r 2}+1\right), & \text { if }\left(U_{r 1}, U_{r 2}\right) \in D_{15} \\ \left(U_{r 1}-1, U_{r 2}-1\right), & \text { if }\left(U_{r 1}, U_{r 2}\right) \in D_{16}\end{cases}
$$

In this way, the value of the marked coefficient pair may be in a new set, which the information could be extracted according to. The value of the coefficient pair could be completely recovered by the reverse process of embedding way. The embedding capacities of the presented 2D HS and the conventional 2D HS, denoted as $E C_{p r e}$ and $E C_{c o n}$, can be computed by (17) and (18).

$$
\begin{aligned}
& E C_{p r e}=2 \sum_{\left(U_{r 1}, U_{r 2}\right) \in D_{1} \cup D_{2}} t\left(U_{r 1}, U_{r 2}\right)+\sum_{\left(U_{r 1}, U_{r 2}\right) \in D_{3} \cup D_{4} \cup D_{5} \cup D_{6} \cup D_{7} \cup D_{8} \cup D_{9} \cup D_{10} \cup D_{11} \cup D_{12}} t\left(U_{r 1}, U_{r 2}\right) \\
& E C_{c o n}=2 \sum_{\left(U_{r 1}, U_{r 2}\right) \in D_{1} \cup D_{2} \cup D_{3} \cup D_{4}} t\left(U_{r 1}, U_{r 2}\right)+\sum_{\left(U_{r}, U_{r 2}\right) \in D_{5} \cup D_{6} \cup D_{7} \cup D_{8} \cup D_{9} \cup D_{10} \cup D_{11} \cup D_{12}} t\left(U_{r 1}, U_{r 2}\right)
\end{aligned}
$$

According to (17) and (18), we can infer the difference of embedding capacity between the presented 2D HS and the conventional 2D HS by

$$
E C_{\text {con }}-E C_{p r e}=\sum_{\left(U_{r 1}, U_{r 2}\right) \in D_{3} \cup D_{4}} t\left(U_{r 1}, U_{r 2}\right)
$$

For QDCT coefficients, the distortion in terms of $l^{2}$-error of the presented 2D HS and the customary 2D HS, denoted as $E D_{\text {pre }}$ and $E D_{\text {con }}$, can be formulated as (20) and (21).

$$
\begin{aligned}
& E D_{p r e}=\frac{3}{4} \sum_{\left(U_{r 1}, U_{r 2}\right) \in D_{1} \cup D_{2}} t\left(U_{r 1}, U_{r 2}\right)+\sum_{\left(U_{r 1}, U_{r 2}\right) \in D_{3} \cup D_{4} \cup D_{5} \cup D_{6} \cup D_{7} \cup D_{8}} t\left(U_{r 1}, U_{r 2}\right) \\
& +\frac{3}{2} \sum_{\left(U_{r 1}, U_{r 2}\right) \in D_{9} \cup D_{10} \cup D_{11} \cup D_{12}} t\left(U_{r 1}, U_{r 2}\right)+2 \sum_{\left(U_{r 1}, U_{r 2}\right) \in D_{13} \cup D_{14} \cup \cup D_{15} \cup D_{16}} t\left(U_{r 1}, U_{r 2}\right)
\end{aligned}
$$




$$
\begin{aligned}
& E D_{\text {con }}=\sum_{\left(U_{r 1}, U_{r 2}\right) \in D_{1} \cup D_{2} \cup D_{3} \cup D_{4}} t\left(U_{r 1}, U_{r 2}\right)+\frac{3}{2} \sum_{\left(U_{r 1}, U_{r 2}\right) \in D_{5} \cup D_{6} \cup D_{7} \cup D_{8} \cup D_{9} \cup D_{10} \cup D_{11} \cup D_{12}} t\left(U_{r 1}, U_{r 2}\right) \\
& +2 \sum_{\left(U_{r}, U_{r 2}\right) \in D_{13} \cup D_{14} \cup D_{15} \cup D_{16}} t\left(U_{r 1}, U_{r 2}\right)
\end{aligned}
$$

Therefore, we can infer the difference of hiding distortion between the presented 2D HS and the conventional 2D HS by

$$
E D_{c o n}-E D_{p r e}=\frac{1}{4} \sum_{\left(U_{r 1}, U_{r 2}\right) \in D_{1} \cup D_{2}} t\left(U_{r 1}, U_{r 2}\right)+\frac{1}{2} \sum_{\left(U_{r 1}, U_{r 2}\right) \in D_{5} \cup D_{6} \cup D_{7} \cup D_{8}} t\left(U_{r 1}, U_{r 2}\right)
$$

According to (19) and (22), compared with the conventional 2D HS, to get the same quantity of embedding information, the distortion caused by our scheme is lower. When the value of the QDCT coefficient pair $\left(U_{r 1}, U_{r 2}\right)$ belongs to the sets $D_{1}, D_{2}, D_{5}, D_{6}, D_{7}$, or $D_{8}$, although the same capacity (two data bits or one data bit) can be gotten by the two methods, 2 is the largest cost in the conventional HS, whereas at most one modification is caused by our method. When $\left(U_{r 1}, U_{r 2}\right)$ belongs to the sets $D_{3}$ or $D_{4}$, if the same number of data is embedded, the same distortion will be caused. When the value of the QDCT coefficient pair $\left(U_{r 1}, U_{r 2}\right)$ belongs to other sets, the same capacity and distortion will be gotten by the two methods.

\section{2D-HS-BASED MVC VIDEO RDH ALGORITHM}

The presented RDH algorithm based on 2D HS for MVC video is shown in Figure 4. For the sender, in order to enhance the security and the robustness of the hidden information, the information is encrypted and encoded by binary $\mathrm{BCH}$ code before data hiding. The original MVC video is entropy decoded to get QDCT MBs, where some MBs are chosen for hiding data, and the unselected MBs will be entropy encoded directly. According to the approach depicted in Figure 3, several data bits are embedded into each embeddable QDCT coefficient pair, which could be randomly selected from every embeddable $4 \times 4$ block in a MB. Therefore, for the receiver, the hidden data could be extracted from the marked QDCT coefficient pairs after entropy decoding. 


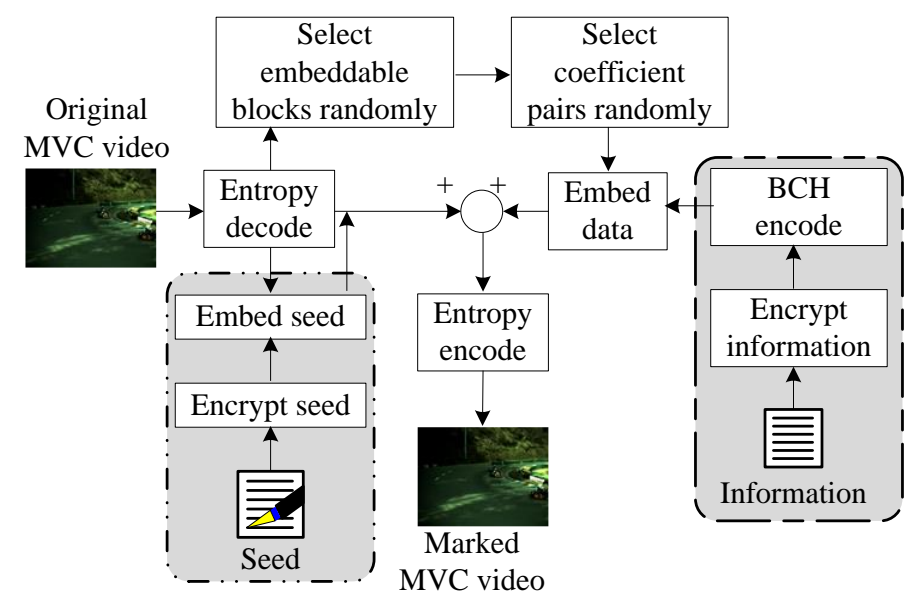

(a)

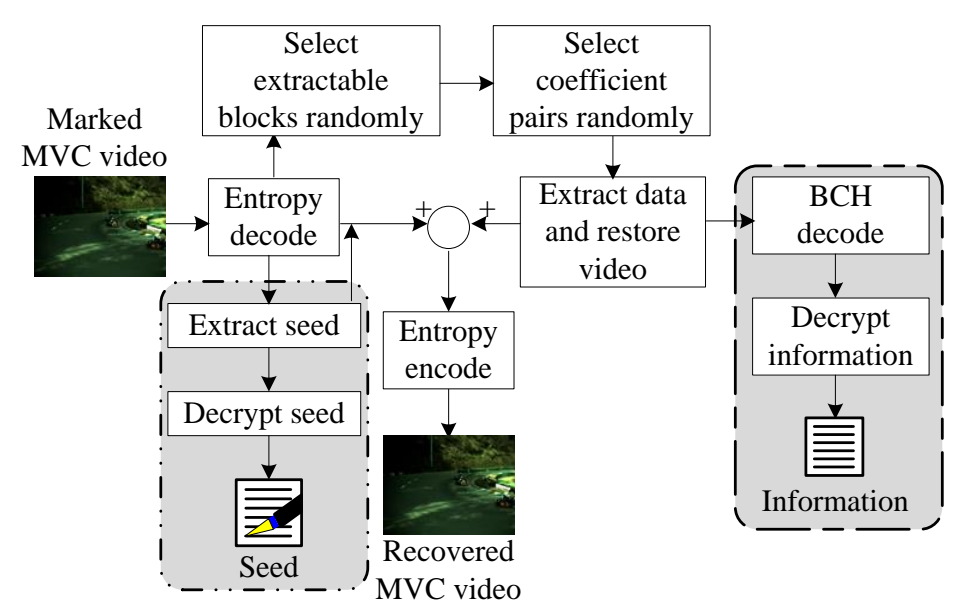

(b)

Figure 4. The flowchart of the presented 2D-HS-based RDH algorithm: (a) Information embedding, (b) Information extraction and carrier recovery

\subsection{Distortion Propagation Preclusion}

Figure 5 illustrates the prediction scheme of MVC video with hierarchical B coding and two views. Horizontal arrows indicate inter-frame prediction, and vertical arrows express parallax prediction. Each group of picture (GOP) includes 16 frames, where there are eight frames in each view. $\mathrm{I}_{0}$ frames and $\mathrm{P}_{0}$ frames are key frames at the highest level marked by 0 . Only intra-frame prediction is used in $\mathrm{I}_{0}$ frames so that they will not be affected by other frames. Whereas the distortion of one $\mathrm{I}_{0}$ frame will affect all the frames in the two adjacent GOPs predicted by this $\mathrm{I}_{0}$ frame. In contrast, parallax distortion drift will be avoided by embedding data into $\mathrm{P}_{0}$ frames, which do not predict the frames in the left view. The parallax and the inter-frame distortion drift will be eliminated by hiding data into $b_{4}$ frames, which are not referred by other frames. But in the internet transfers, it is easy to lose $b_{4}$ frames located at the lowest level. By comparison, stronger robustness can be obtained by hiding data into $\mathrm{P}_{0}$ frames located at the highest level. Additionally, six $\mathrm{B}_{3}$ frames in each GOP could be used for hiding data, where only $\mathrm{b}_{4}$ frames may be changed by hiding data into $B_{3}$ frames. In short, users could select embeddable frames on demand since different frames have strengths and weaknesses. 


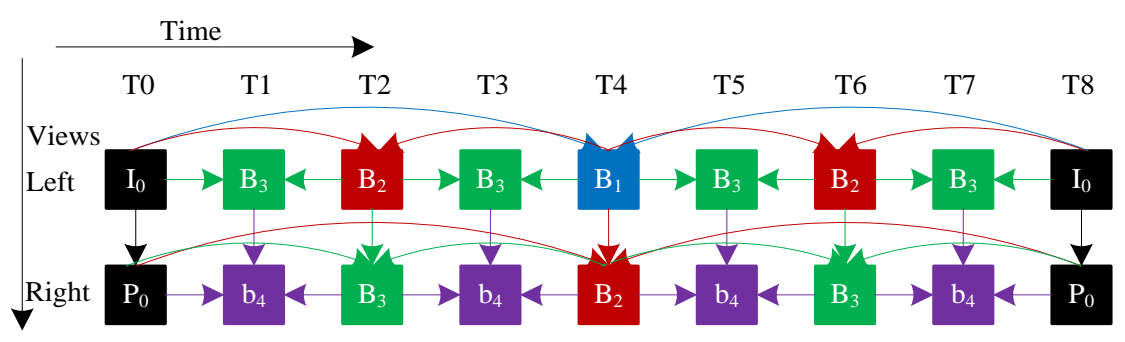

Figure 5. Prediction structure of MVC video with hierarchical B coding and two views

At the MVC encoder, after the prediction block is subtracted from the original pixel block in the YUV video, the consequent residual block, denoted by $L^{R 0}$, undergoes $4 \times 4$ (or $8 \times 8$ ) DCT and quantization as reckoned in:

$$
U=\operatorname{round}\left[\left(C_{f} L^{R 0} C_{f}^{T}\right) \otimes\left(E_{f} / Q\right)\right]
$$

Where $U$ is a QDCT block with 16 QDCT coefficients numbered by zigzag scan, $C_{f}=\left[\begin{array}{cccc}1 & 1 & 1 & 1 \\ 2 & 1 & -1 & -2 \\ 1 & -1 & -1 & 1 \\ 1 & -2 & 2 & -1\end{array}\right], E_{f}=\left[\begin{array}{cccc}a^{2} & a c / 2 & a^{2} & a c / 2 \\ a c / 2 & c^{2} / 4 & a c / 2 & c^{2} / 4 \\ a^{2} & a c / 2 & a^{2} & a c / 2 \\ a c / 2 & c^{2} / 4 & a c / 2 & c^{2} / 4\end{array}\right], a=1 / 2, c=\sqrt{2 / 5}, \quad$ the matrix $C_{f}^{T}$ is the transpose of $C_{f}, Q$ is the quantization step size, $\otimes$ means a mathematical operation, which expresses that each element in the preceding matrix is multiplied by the value at the corresponding position in the latter matrix.

If data is hidden into one QDCT block $U$ by changing some QDCT coefficients, the block $U$ will be altered to a marked QDCT block denoted by $U^{\prime}$, and the deviation (denoted by $\Delta U$ ) caused by hiding data is

$$
\Delta U=U^{\prime}-U
$$

At the decoder, the residual pixel block, denoted as $L^{R}$, is gained by lossy decompression (inverse quantization and $4 \times 4$ inverse DCT) as shown in:

$$
L^{R}=\operatorname{round}\left[C_{u}^{T}\left(U \otimes E_{u}\right) C_{u}\right]
$$

Where $C_{u}=\left[\begin{array}{cccc}1 & 1 & 1 & 1 \\ 1 & 1 / 2 & -1 / 2 & -1 \\ 1 & -1 & -1 & 1 \\ 1 / 2 & -1 & 1 & -1 / 2\end{array}\right], E_{u}=\left[\begin{array}{cccc}a^{2} & a c & a^{2} & a c \\ a c & c^{2} & a c & c^{2} \\ a^{2} & a c & a^{2} & a c \\ a c & c^{2} & a c & c^{2}\end{array}\right]$.

If data is hidden by changing some QDCT coefficients, the residual pixel block $L^{R}$ will be turned into the marked pixel block denoted as $L^{R^{\prime}}$, and the mutation denoted as $\Delta L^{R}$ is

$$
\Delta L^{R}=L^{R^{\prime}}-L^{R}=\operatorname{round}\left[C_{u}^{T}\left(\Delta U \cdot Q \otimes E_{u}\right) C_{u}\right]
$$


Take the QDCT coefficient $U_{15}$ as an instance to prove the distortion caused by hiding data. Assume an integer denoted as $z$ is added to the value of $U_{15}$, i.e., the change of the QDCT block

for hiding data is

$$
\Delta U=\left[\begin{array}{llll}
0 & 0 & 0 & 0 \\
0 & 0 & 0 & 0 \\
0 & 0 & 0 & 0 \\
0 & 0 & 0 & z
\end{array}\right] \text {, then the difference of the corresponding pixel block in }
$$

YUV video is

$$
\Delta L^{R}=\frac{1}{4} Q c^{2} z\left[\begin{array}{cccc}
1 & -2 & 2 & -1 \\
-2 & 4 & -4 & 2 \\
2 & -4 & 4 & -2 \\
-1 & 2 & -2 & 1
\end{array}\right]
$$

In order to hide information, only $z$ is added to the QDCT coefficient $U_{15}$, but the whole $4 \times 4$ pixel block in the corresponding YUV video is altered. Similarly, the modification of one QDCT coefficient in an $8 \times 8$ transformation block will change the whole $8 \times 8$ pixel block, whose distortion range is bigger than $4 \times 4$ block. Additionally, only two kinds of transformations, $4 \times 4$ transformation and $8 \times 8$ transformation, are used in MVC standard. Thus a $4 \times 4$ transformation block is chosen to hide information in this paper.

Accordingly, the boundary pixels denoted as $o_{0}, o_{1}, \ldots, o_{12}$, shown in Figure 6 (In mode 2 that is not depicted in Figure 6(b) and Figure 6 (c), all elements are predicted with the average of upper pixels denoted as $\mathrm{H}$ and left pixels denoted as $\mathrm{V}$, i.e., Mean $(\mathrm{H}+\mathrm{V})$ ), may be changed by embedding data into some QDCT coefficients of the four blocks denoted by $L_{x, y-1}, L_{x-1, y-1}, L_{x-1, y}$, and $L_{x-1, y+1}$ (integers $x$ and $y$ present the position of a block). If intra-frame prediction is employed in the current block $L_{x, y}$, its prediction block will be calculated by the pixels $o_{0} \ldots o_{12}$. So the distortion of the blocks $L_{x, y-1}, L_{x-1, y-1}, L_{x-1, y}$, and $L_{x-1, y+1}$ will drift to the block $L_{x, y}$. Otherwise, if parallax prediction or inter-frame prediction is applied in the block $L_{x, y}$, whose prediction block is gotten by referring other frames as shown in Figure 5, the block $L_{x, y}$ will not be affected by any modification of its neighboring blocks.

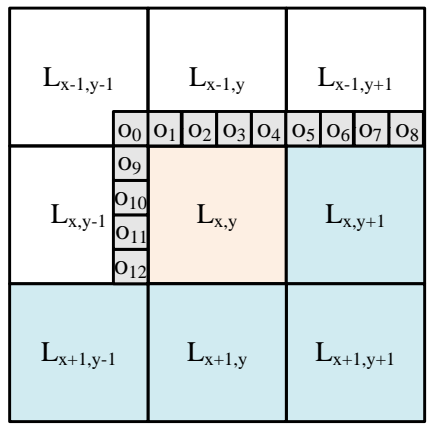

(a)

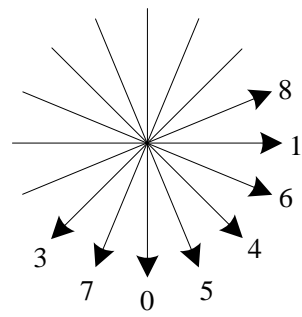

(b)

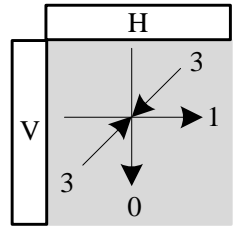

(c)

Figure 6. Intra-frame prediction modes: (a) Position, (b) Predictive directions of $4 \times 4$ blocks, (c) Predictive directions of $16 \times 16$ blocks.

Let inter-MB be a $16 \times 16 \mathrm{MB}$ with inter-frame prediction or parallax prediction. The nine $4 \times 4$ blocks numbered by $0 \ldots 8$ are not located at the rightmost column or the bottom row of the interMB shown in Figure 7. If the block $L_{x, y}$ is such a block, its neighboring blocks $L_{x, y+1}, L_{x+1, y+1}$ 
and $L_{x+1, y}$ will be in the current inter-MB. In addition, the neighboring block $L_{x+1, y-1}$ may be in either the current inter-MB or one of the blocks numbered by $9 \ldots 11$ in the encoded MB at the encoder (or a decoded MB at the decoder). Thus these adjacent blocks $L_{x, y+1}, L_{x+1, y+1}, L_{x+1, y}$ and $L_{x+1, y-1}$ will not be affected by the current block $L_{x, y}$. Therefore, the nine $4 \times 4$ blocks numbered by $0 \ldots 8$ in each inter-MB can be chosen as embeddable blocks for hiding data without intra-frame distortion drift.

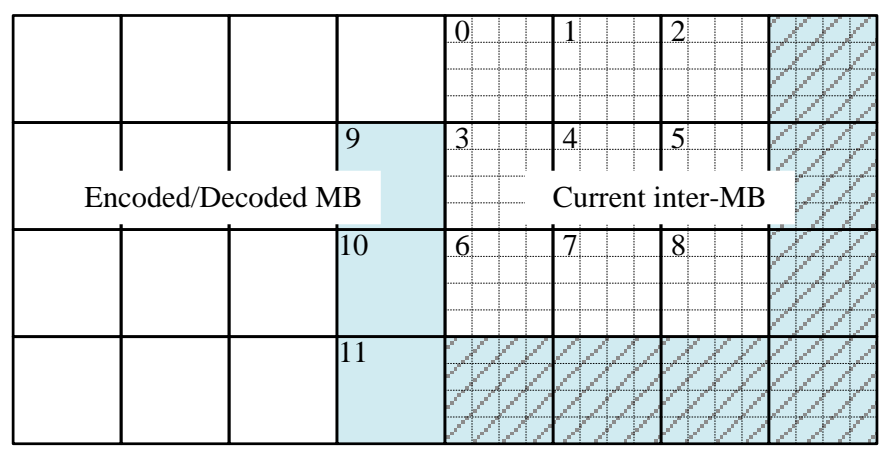

Figure 7. $4 \times 4$ embeddable blocks without intra-frame distortion drift

\subsection{Data Embedding}

The embedding flowchart at the encoder is shown in Figure 4(a). There are five main steps to complete the data-hiding process: processing the to-be-hidden information, embedding the seed denoted by $K$, choosing embeddable blocks, selecting coefficient pairs, and data embedding.

A. Processing the to-be-hidden information To ensure safety, the original information is encrypted into cypher text with a RSA public key. Then the encrypted information is encoded by $\mathrm{BCH}$ code before data hiding to guarantee the robustness.

B. Embedding the seed $\mathbf{K}$ To improve the security, random function and the seed Kare used to select embeddable blocks and coefficients. We encrypt the seed $\mathrm{K}$ with a RSA public key and hide the seed $\mathrm{K}$ into some QDCT MBs of $\mathrm{I}_{0}$ frames with a public RDH algorithm.

C. Choosing embeddable blocks It is necessary to limit the distortion region since large region of distortion is obvious. Random block numbers are generated to select embeddable $4 \times 4$ blocks (not at the bottom row or the rightmost column, as shown in Figure 7), which could be used for hiding data without intra-frame distortion drift. Additionally, a positive integer is set to generate a random threshold denoted by $\mathrm{G}$ so that embeddable blocks could be chosen randomly according to $\left|\mathrm{U}_{0}\right| \geq \mathrm{G}$. When the threshold $\mathrm{G}$ is bigger, the fewer embeddable blocks will be found, and the region of the distortion will be less.

D. Selecting coefficient pairs Two QDCT coefficients $\left(\mathrm{U}_{\mathrm{r} 1}, \mathrm{U}_{\mathrm{r} 2}\right)$ are randomly chosen from each embeddable blocks. There are 15 ways to choose $\mathrm{U}_{\mathrm{r} 1}$ from $15 \mathrm{AC}$ coefficients, and 14 ways to select $\mathrm{U}_{\mathrm{r} 2}$ from the rest 14 AC coefficients. Thus the optional of selecting $\left(\mathrm{U}_{\mathrm{r} 1}, \mathrm{U}_{\mathrm{r} 2}\right)$ is $15 \times 14=210$. Given a marked block, the probability for directly guessing the hidden data bit is $1 / 210 \approx 0.00476$. In this way, the application of arbitrary embedding positions including blocks and coefficients can be used to reduce the modification of statistical histogram and improve the undetectability of RDH algorithm.

E. Data embedding Some data bits are hidden into each coefficient pair by following Figure 3. 


\subsection{Data Extracting and Video Restoring}

Figure 4(b) displays the procedure of information extraction and video restoration. There are four main steps to complete this process: extracting the seed $K$, choosing extractable blocks and coefficient pairs, data extracting and video restoring, processing the extracted information.

A. Extracting the seed K After the entropy decoding of the MVC video, we extract the seed $\mathrm{K}$ from $\mathrm{I}_{0}$ frame with the public $\mathrm{RDH}$ algorithm and decrypt the seed $\mathrm{K}$ with the RSA private key.

B. Choosing extractable blocks and coefficient pairs The same random seed can be used to generate the same random sequence. Thus the communication parties can use the same random seed $\mathrm{K}$ to choose the same embeddable/ extractable blocks and coefficient pairs, where information could be hidden without intra-frame distortion drift.

C. Data extracting and video restoring According to the reverse process of Figure 3, we can extract the hidden information and completely recover the MVC video.

D. Processing the extracted information Finally, we use $\mathrm{BCH}$ code technique to recover the information and decrypt the hidden data.

\section{EXPERIMENTAL RESULTS AND DISCUSSION}

The presented algorithm has been effectuated in the H.264 reference software version JM18. $4[21]$. Test samples are the nine video sequences [22] (the size of each frame is $640 \times 480$ ) shown in Figure 8. Two YUV files are encoded into a MVC video with 233 frames (at 30 frames/second) and two views, left view and right view, which has 30 P0 frames. The parameters intra-period and quantization step are set to 8 and 28, respectively. The capacity of a sequence is the average number of bits embedded into each $\mathrm{P} 0$ frame of the $30 \mathrm{P} 0$ frames in that sequence. The peak signal-to-noise ratio (PSNR) value compared to the original YUV files is the average of all the frames in two views. The structural similarity (SSIM) compared to the original YUV files is the average of all the frames. The embedding efficiency e is defined as

$$
e=N_{\text {hide }} / \sum_{i=1}^{i=N_{m}} L m_{i}
$$

where $N_{\text {hide }}$ is the number of embedded bits, $L m_{i}$ is the changed size of the $i$ th modified QDCT coefficient, $N_{m}$ is the number of modified QDCT coefficients.

\subsection{Data Hiding Performance}

The capacity, PSNR, SSIM, bit-rate increase and embedding efficiency always depend on the video content and the embeddable block selection conditions. First, different embedding performances can be achieved by hiding data into different videos. Second, lower embedding capacity, better video quality, lower bit-rate increase, and less embedding efficiency can be obtained by data hiding with stricter block selection condition (higher threshold). When the threshold $G$ increases, the increases in PSNR and SSIM values are very gentle, whereas the embedding capacity, the bit-rate increase and the embedding efficiency decline sharply. 


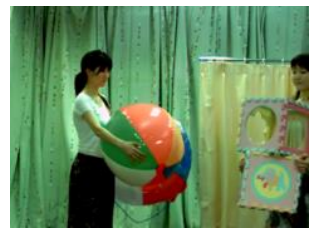

(a)

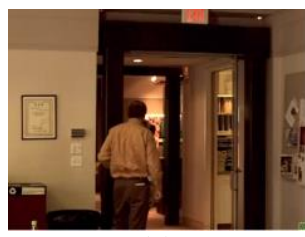

(d)

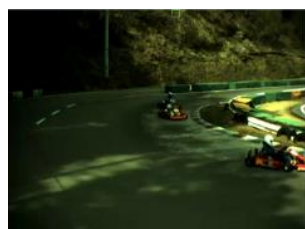

(g)

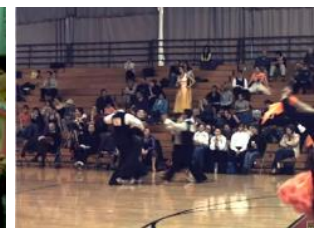

(b)

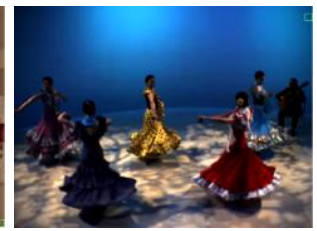

(e)

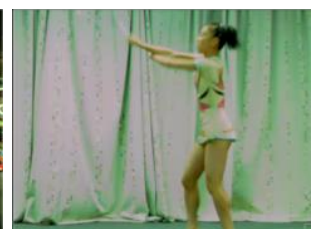

(h)

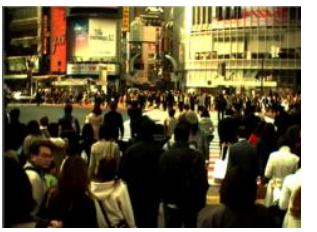

(c)

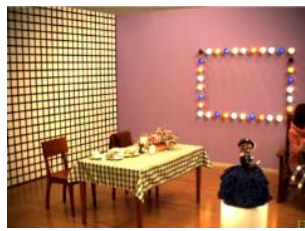

(f)

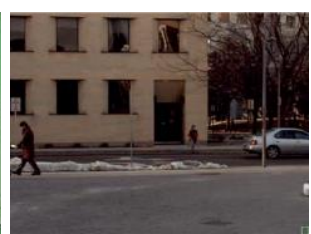

(i)

Figure 8. Test videos (the size of each frame is 640×480) (a) Akko\&Kayo, (b) Ballroom, (c) Crowd, (d) Exit, (e) Flamenco, (f) Objects, (g) Race, (h) Rena and (i) Vassar

Figure 9 shows the performance comparison of four different RDH algorithms. In the method presented in [17] denoted by DE, the algorithm presented in [1] denoted by PS, and the proposed scheme, data is hidden into QDCT coefficient pair $\left(\mathrm{U}_{2}, \mathrm{U}_{5}\right)$, where $\mathrm{U}_{2}$ is used by [14], denoted by 1DHS.

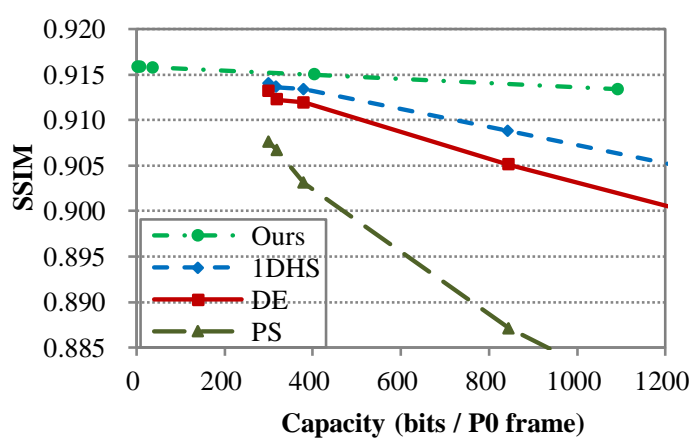

(a) Vassar

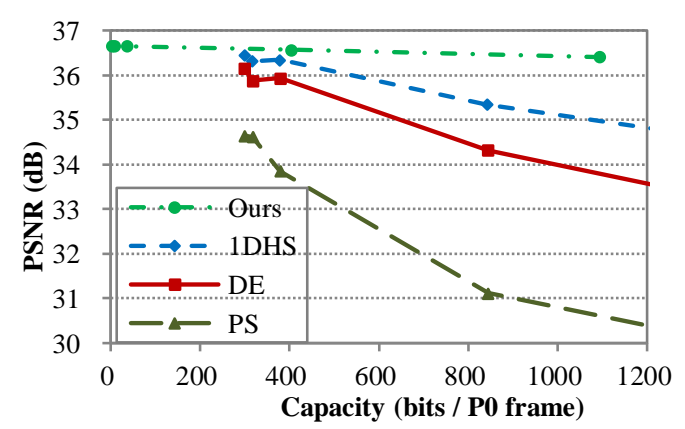

(c) Vassar

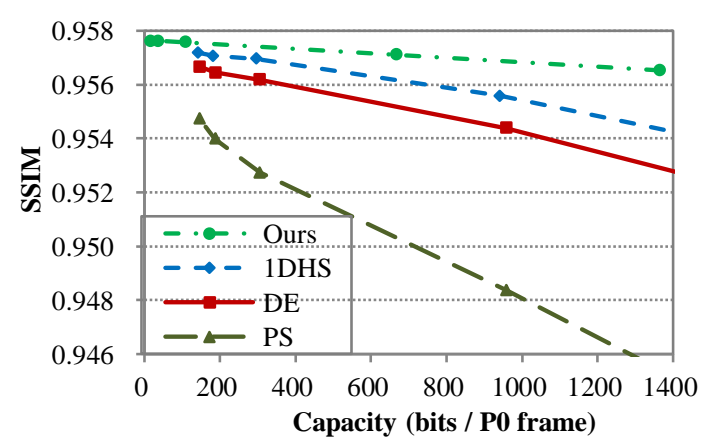

(b) Average of 9 videos

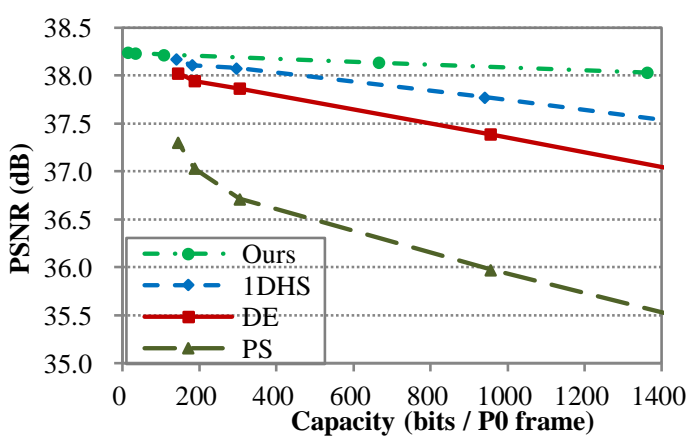

(d) Average of 9 videos 


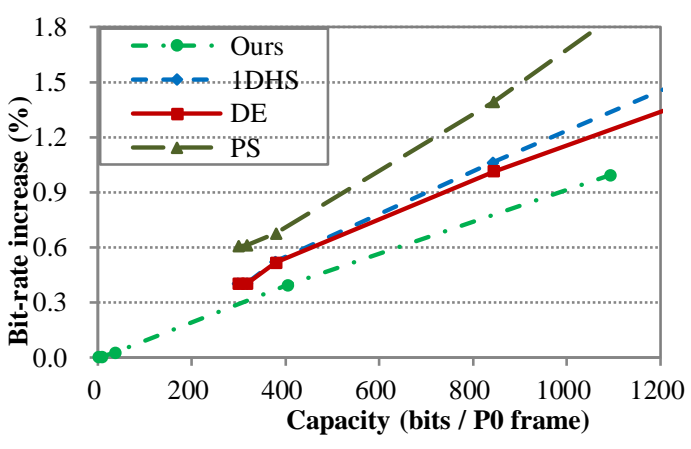

(e) Vassar

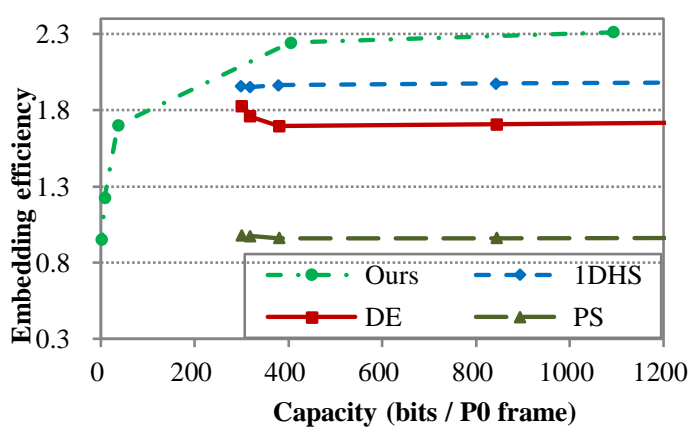

(g) Vassar

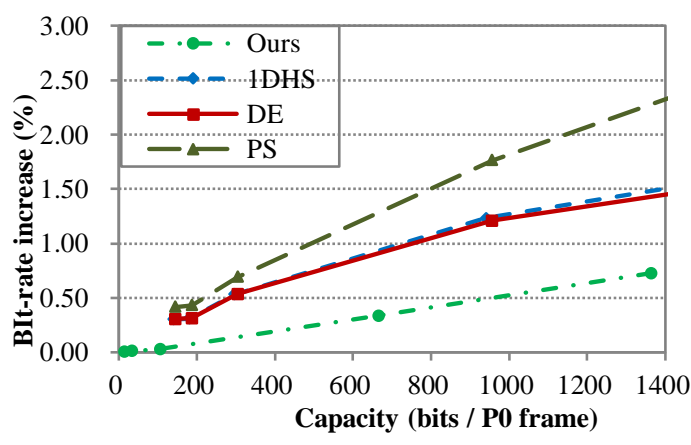

(f) Average of 9 videos

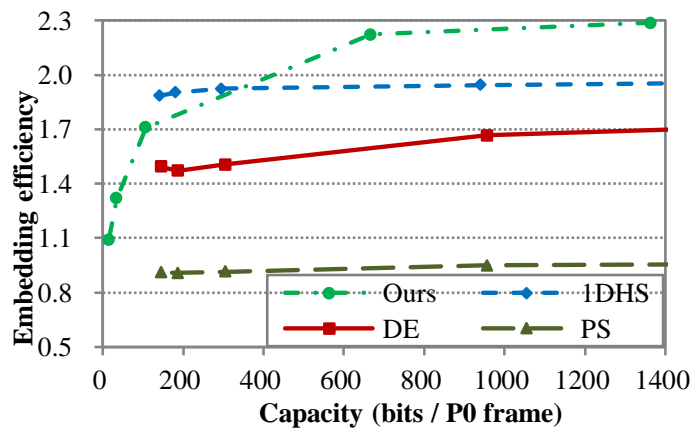

(h) Average of 9 videos

Figure 9. Comparison of hiding performance on different schemes

The experimental results show that when the same number of data is hidden, the best SSIM, PSNR, embedding efficiency, and the least bit-rate increase can be obtained by using the presented algorithm. The best SSIM and PSNR show that the best video quality can be gained by using our scheme. The highest embedding efficiency means that if the same modification is made, the most data bits could be hidden by using our method. According to the lower bit-rate increase, we can know that the application of the proposed method has minimal impact on the coding efficiency of MVC.

In order to further compare these methods, the threshold $G$ is set to be 0 and 1000 bits of data are hidden into one $\mathrm{P}_{0}$ frame of Race on average, as shown in Table 1. Compared with some state-ofthe-art RDH methods, our RDH algorithm is superior by enhancing PSNR, SSIM, and embedding efficiency with $0.19 \mathrm{~dB}, 0.0007$, and 0.5 at least, respectively.

Table 1. Comparison of embedding performance between the proposed algorithm and four methods for hiding 1000 bits of information into one P0 frame of Race on average

\begin{tabular}{|l|l|l|l|l|}
\hline & Ours & 1DHS & DE & PS \\
\hline PSNR(dB) & 37.62 & 37.43 & 37.38 & 36.87 \\
\hline SSIM & 0.9614 & 0.9607 & 0.9605 & 0.9593 \\
\hline e & 2.21 & 1.71 & 1.55 & 0.95 \\
\hline
\end{tabular}

Accordingly, parts of the marked frames of Race are shown in Figure 10. It is easy to find apparent distortion on the trees in the frame (b). By contrast, little distortion could be seen in the 
frame (c). The experimental results verify that superior visual quality can be achieved by using the proposed method to embed information.

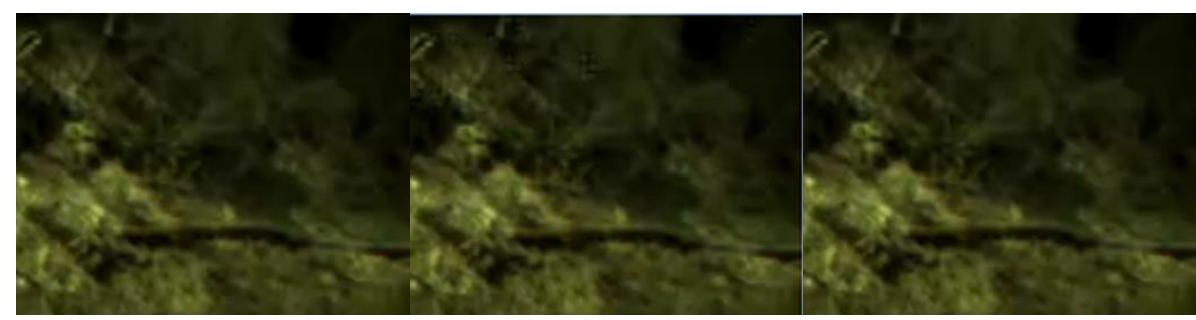
(a) Original
(b) $1 \mathrm{DHS}$
(c) Proposed scheme

Figure 10. The marked frames of Race generated by 1DHS and the proposed 2D HS with 1000 bits of information

\subsection{Discussions}

The computational efficiency of the proposed RDH scheme is related to the frame number denoted by $L_{V}$ and the information length $N_{\text {hide }}$, so the computational complexity of the proposed algorithm could be denoted as $\mathrm{O}\left(L_{V} \times N_{\text {hide }}\right)$. All the experiments were run on a machine with 24 processors $(2.5 \mathrm{GHz})$ and $32 \mathrm{~GB}$ of RAM. It took $162 \mathrm{~ms}$ on average to run the data hiding procedure for each frame of the test videos. The video decoding procedure without data hiding for each frame of the test videos cost $161 \mathrm{~ms}$ on average. The duration caused by hiding data for each frame is $1 \mathrm{~ms}$ on average. Therefore, the presented method with low complexity can be used for real-time application.

Furthermore, in our paper [23], we have tested that $\operatorname{BCH}(7,4,1)$ is the most powerful code in terms of error correction capability if 1 random error bit is corrected. Otherwise, $\mathrm{BCH}(63,7,15)$ is the most powerful code in terms of error correction capability, where the hidden data bits can be recovered $100 \%$ when the frame loss rate is no more than $15 \%$. So high robustness could be achieved by using the proposed scheme shown in Figure 4.

\section{Conclusions}

A novel 2D HS is proposed in this paper. Utilizing this scheme, two data bits can be hidden with at most one modification, whereas two will be cost in the conventional HS. Compared with some state-of-the-art methods, the proposed scheme has superior hiding performance. In our future work, we will generalize the proposed scheme for hiding over 2 bits of data with at most one modification, and extend this method to image RDH such as difference HS and prediction-error HS.

\section{ACKNOWLEDGMENT}

This work is supported by the Natural Science Foundation of Hubei Prorince under Grant 2017 CFB306 .

The authors are heartily grateful to the reviewers for their valuable comments improving the quality of the original manuscript. 


\section{REFERENCES}

[1] S. Maiti and M. P. Singh, "A novel reversible data embedding method for source authentication and tamper detection of H.264/AVC video," presented at the 5th International Conference on Information Processing, ICIP 2011, Bangalore, India, August 5, 2011 - August 7, 2011, 2011. Available: <Go to ISI>://WOS:000306579700044

[2] K. L. Chung, Y. H. Huang, P. C. Chang, and H. Y. M. Liao, "Reversible data hiding-based approach for intra-frame error concealment in H.264/AVC," (in English), IEEE Transactions on Circuits and Systems for Video Technology, vol. 20, no. 11, pp. 1643-1647, Nov 2010.

[3] D. W. Xu, R. D. Wang, and Y. Q. Shi, "An improved reversible data hiding-based approach for intraframe error concealment in H.264/AVC," (in English), Journal of Visual Communication and Image Representation, Article vol. 25, no. 2, pp. 410-422, Feb 2014.

[4] J. Zhao, Z. Li, and B. Feng, "A novel two-dimensional histogram modification for reversible data embedding into stereo H.264 video," Multimedia Tools and Applications, vol. 75, no. 10, pp. 59595980, May 2016.

[5] J. Zhao and Z. Li, "Three-dimensional histogram shifting for reversible data hiding," Multimedia Systems, vol. 24, no. 1, pp. 95-109, Feb 2018.

[6] Z. C. Ni, Y. Q. Shi, N. Ansari, and W. Su, "Reversible data hiding," (in English), IEEE Transactions on Circuits and Systems for Video Technology, Article vol. 16, no. 3, pp. 354-362, Mar 2006.

[7] W. F. Qi, X. L. Li, T. Zhang, and Z. M. Guo, "Optimal Reversible Data Hiding Scheme Based on Multiple Histograms Modification," Ieee Transactions on Circuits and Systems for Video Technology, vol. 30, no. 8, pp. 2300-2312, Aug 2020.

[8] C.-C. Lin, W.-L. Tai, and C.-C. Chang, "Multilevel reversible data hiding based on histogram modification of difference images," Pattern Recognition, vol. 41, no. 12, pp. 3582-3591, Dec 2008.

[9] L. L. An, X. B. Gao, X. L. Li, D. C. Tao, C. Deng, and J. Li, "Robust reversible watermarking via clustering and enhanced pixel-wise masking," IEEE Transactions on Image Processing, vol. 21, no. 8, pp. 3598-3611, Aug 2012.

[10] X. L. Li, B. Li, B. Yang, and T. Y. Zeng, "General framework to histogram-shifting-based reversible data hiding," (in English), IEEE Transactions on Image Processing, Article vol. 22, no. 6, pp. 21812191, Jun 2013.

[11] C. Qin, C. C. Chang, Y. H. Huang, and L. T. Liao, "An inpainting-assisted reversible steganographic scheme using a histogram shifting mechanism," (in English), IEEE Transactions on Circuits and Systems for Video Technology, Article vol. 23, no. 7, pp. 1109-1118, Jul 2013.

[12] H. Yao, F. Y. Mao, Z. J. Tang, and C. Qin, "High-fidelity dual-image reversible data hiding via prediction-error shift," Signal Processing, vol. 170, May 2020, Art. no. 107447.

[13] B. Ou and Y. Zhao, "High Capacity Reversible Data Hiding Based on Multiple Histograms Modification," Ieee Transactions on Circuits and Systems for Video Technology, vol. 30, no. 8, pp. 2329-2342, Aug 2020.

[14] X. Z. Xie, C. C. Chang, and Y. C. Hu, "An adaptive reversible data hiding scheme based on prediction error histogram shifting by exploiting signed-digit representation," Multimedia Tools and Applications, vol. 79, no. 33-34, pp. 24329-24346, Sep 2020.

[15] J. Tian, "Reversible data embedding using a difference expansion," (in English), IEEE Transactions on Circuits and Systems for Video Technology, Article vol. 13, no. 8, pp. 890-896, Aug 2003.

[16] Z. W. Zhang, M. J. Zhang, and L. Y. Wang, "Reversible Image Watermarking Algorithm Based on Quadratic Difference Expansion," Mathematical Problems in Engineering, vol. 2020, Jan 2020, Art. no. 1806024.

[17] C. Y. Weng, "DWT-based reversible information hiding scheme using prediction-error-expansion in multimedia images," Peer-to-Peer Networking and Applications, vol. 13, no. 2, pp. 514-523, Mar 2020.

[18] W. G. He and Z. C. Cai, "An Insight Into Pixel Value Ordering Prediction-Based Prediction-Error Expansion," Ieee Transactions on Information Forensics and Security, vol. 15, pp. 3859-3871, 2020.

[19] D. Coltuc, "Low distortion transform for reversible watermarking," IEEE Transactions on Image Processing, vol. 21, no. 1, pp. 412-417, Jan 2012.

[20] Y. Q. Qiu, Z. X. Qian, H. Q. Zeng, X. D. Lin, and X. P. Zhang, "Reversible data hiding in encrypted images using adaptive reversible integer transformation," Signal Processing, vol. 167, Feb 2020, Art. no. 107288 . 
[21] K. Sühring. (Aug). H.264/AVC software coordination (JM 18.4 ed.). Available: http://iphome.hhi.de/suehring/tml

[22] (Feb). Video test sequences. Available: http://blog.csdn.net/do2jiang/article/details/5499464

[23] Y. Liu, Z. Li, X. Ma, and J. Liu, "A robust without intra-frame distortion drift data hiding algorithm based on H.264/AVC," Multimedia Tools and Applications, vol. 72, no. 1, pp. 613-636, Sep. 2014.

\section{AUTHORS}

Juan Zhao received her B.S. degree from Henan Normal University, Xinxiang, China, in 2007, and PhD degree from Huazhong University of Science and Technology, Wuhan, China, in 2015. She is currently a lecturer in School of Mathematics \&Computer Science, Wuhan Polytechnic University. Her research interests include data hiding, network security and multimedia security.

Zhitang Li received his M.E. degree in Computer Architecture from Huazhong University of Science and Technology, Wuhan, China, 1987, and $\mathrm{PhD}$ degree in Computer Architecture from Huazhong University of Science and Technology, Wuhan, China, 1992. His research interests include computer architecture, network security, and P2P networks. He was the director of China Education and Research Network in Central China. He was a vice president of Department of Computer Science and Technology, Huazhong University of Science and Technology, China. He has published more than one hundred papers in the areas of network security, computer architecture, and P2P networks.

(C) 2021 By AIRCC Publishing Corporation. This article is published under the Creative Commons Attribution (CC BY) license. 


\title{
Cell Switches Model Applying MARKov CHAIN STOCHASTIC MODEL CHECK ON BETWEEN TWO POPULATION WITH REGARDS TO MRNA AND PROTEINS AND NEURONS BOTH CLASSICALLY AND QUANTUM COMPUTATIONALLY
}

\author{
Qin He, Rubin Wang and Xiaochuan Pan \\ East China University of Science and Technology, Meilong 130, China
}

\begin{abstract}
Arc, one virus-like gene, crucial for learning and memory, was dis-covered by researchers in neurological disorders fields, Arc mRNA's single directed path and allowing protein binding regional restric-tively is a potential investigation on helping shuttle toxic proteins responsible for some diseases related to memory deficiency. Mean time to switching (MTS) is calculated explicitly quantifying the switching process in statistical methods combining Hamiltonian Markov Chain $(H M C)$. The model derived from predator and prey with typeII functional response studies the mechanism of normals with intrin-sic rate of increase and the persisters with the instantaneous discovery rate and converting coefficients. During solving the results, since the numeric method is applied for the $2 D$ approximation of Hamiltonion with intrinsic noise induced switching combining geometric minimum action method. In the application of Hamiltonian Markov Chain, the behavior of the convertion (between mRNA and proteins through 6 states from off to on) is described with probabilistic conditional logic formula and the final concentration is computed with both Continuous and Discret Time Markov Chain(CTMC/DTMC) through Embedding and Switching Diffusion. The MTS, trajectories and Hamiltonian dynamics demonstrate the practical and robust advantages of our model on interpreting the switching process of genes (IGFs, Hax Arcs and etc.) with respects to memory deficiency in aging process which can be useful in further drug efficiency test and disease curing. Coincidentally, the Hamiltonian is also well used in describing quantum mechanics and convenient for computation with time and position information using quantum bits while in the second model we construct, switching between excitatory and inhibitory neurons, similarity of qubit and neuron is an interesting object as well. Especially with the interactions operated with phase gates, the excitation from the ground state to excitation state is a well analogue to the neuron excitation. Not only on theoretical aspect, the experimental methods in neuron switching model is also inspiring to quantum computing. Most basic one is as stimulate hippocampus can be identical to spontaneous neural excitation $(|g>| e>)$, pi-pulse is utilized to drive the ground state to the higher state. There thus exists prosperous potential to study the transfer between states with our switch models both classical and quantum computationally.
\end{abstract}

\section{KEYWORDS}

switching model, mean time to switching, Hamiltonian Markov Chain, geometric minimum action method, firing rate, neuron models, Hopfield network, excitation and inhibition, quantum computation, fast gates, phase estimation, sweep entanglement. 


\section{INTRODUCTION}

In cell biology, non-equilibrium stochastic process is of interest since the observation of experimental results are becoming of higher resolution, studying the molecules both with imaging and expression data are often conducted in both single and population (thousand) order, which basically described in stochastic process whether on a discrete or continuous scale with status changes either genotypically or phenotypically. Many problems are thus studied related to status switching, including cell regulatory networks, signal response on excitability and inhibition [1], (convinced by translational and transcriptional burst of expression for instances.), metastability among populations, (binding of ligands and proteins, forming of polymerases and etc.).In this paper, we first focus on the interaction among genes, mRNA, proteins and etc. To be more specific, while the switching problem among molecules can be studied on geno-type, including sequencing for single RNA, alignments and binding considering condons and etc, we stay on the switching with expression (concentration) only, which is simplified as modified population problem using Lotka-Volterra equations[3] of two populations only. Thus, rather than the competitor model (for instances, cell bifurcations.), we applied simulation of switching on predator model. The model is based on the following basic assumptions: Prey population (promoters) is fed with enough food all the time while the predator population of the predator (the persisters) depends on the size of prey (promoters).

To be more specific, while the switching problem among molecules can be studied on geno-type, including sequencing for single RNA, alignments and binding considering condons and etc, we stay on the switching with expression (concentration) only, which is simplified as modified population problem using Lotka-Volterra equations[3] of two populations only. Thus, rather than the competitor model (for instances, cell bifurcations.), we applied simulation of switching on predator model. The model is based on the following basic assumptions: Prey population (promoters) is fed with enough food all the time while the predator population of the predator (the persisters) depends on the size of prey(promoters).

In the next part, we focus on the interpretation of the working memory modelled through neuron interactions. Due to the slow oscillation, there occurs the up-state and down-state [4]. The solution to the differential equation is initialized on the two formula with regards to intrinsic oscillation $\mathrm{dS} / \mathrm{st}$ and evolution of phase $\mathrm{d} \varphi / \mathrm{dt}$ respectively. The reduce to the normal form is completed with combination of firing model and leakage-gate model [5]. The parameters are solved analytically first and numerically simulated with experiment data.

In practical, for the first model. we mainly study the interaction of DNA and its interaction with the associated proteins.(Clinical data of Hax1 and HS1 is downloaded from Ensmbl gene database[2]). On one hand, the switching model is calculated under the large deviation theory (LDT) [5] combining the least actions. The Markov chain[6] consider the states of the 2D coordinates $(\mathrm{x} ; \mathrm{y})$ of mRNA numbers and protein numbers referencing the distribution of $\mathrm{x}$, which follows the order $\mathrm{O}(1)$ while $\mathrm{PX}$ follows the time scale on $\mathrm{O}(1 / \mathrm{e})$ and guaran-teeing the variant of LDT hold with the transform of the expressions in single population. Only considering the process of diffusion case, we study the binding of hax 1 with simple switching between on and off status under its interaction with HS1 seen as in the constant environment, i.e. the closed system at mean field. The dimer which can be cancelled out connect the binding between two single population. On the other hand, one numeric method is applied to solve the problem, making compare with the stochastic process[7] on ap-proximation equation of the mean switching time(MST) with the transform between two status (we studied the switching time with four situations, both multiplicative and asymptotic of single population and the binding and degradation between two population.) Again, this method is also calculated based on the Hamiltonians. We give out the MST with respect to $\mathrm{N} / \mathrm{Nc}$ denoting $\mathrm{N}$ as the population number 
of interest and $\mathrm{Nc}$ as the threshold of certain status (either of that population or the other population). Since our study only based on data in the process of transforming in the constant environment, extinction is not considered in this paper.

On contrary, the second model applies the deterministic model with the single unit model based on normal equation reduced after frequency transfer with firing rate model and leakage model on the relation between I and V. Similar as the first model, eigenvalues are solved with Jacobian matrix at the fixed stable point. This part gives the core algorithm as well. It represents the populations of NMDA, AMPA, GABA cells, separating the system into interacting networks [8]: Positive Network Task (PNT) and Negative Network Task (NNT). In PNT, the excitatory population AMPA and inhibitory population GABA interacted mutually while in NNT, the excitatory population NMDA interacts with inhibitory GABA. The connection in each population and each unit are all assumed to be bidirection with the weight difference only across populations. Similarly, the weight cross units are also different as neuron cannot connect from inhibitory population to excitatory population across unit, the weight accordingly can be seen as zero. And the global feedback of inhibitory network is thus constructed [9]. And in aim of exploring the transfer between states from another aspect, quantum computation is introduced as well. From the Hamiltonian as well, the interactions are studied on energy/power spectrum level clearly with not only related to time but also to the dynamics comprehensively. Due to comparison with classical switch model, we focus on the internal interactions as well. Thus, the switching phase analysis between ground state $|g\rangle$ and higher state $|e\rangle$ or Stark eigenstate $|r\rangle$ were introduced. In the illustration of entanglement, the sweep operation [10] is chosen as its advantage of flexible and competitive. The fixed point finding in classical computation is adopted into the eigenstate define in quantum computation and here is exhibited with detail as well. More operation and demonstration with regards to Bell states/ GHZ [11] is also interesting although not the focus in this paper.

To study both intrinsic and extrinsic noise with the exciting and inhibiting bursts is the potential topic in the future. In the following contents, the first chapter is the proposition of the model, based on least action with LDT and MTS approximation with one stochastic differential equation (SDE) [13]separately; And the second chapter gives numeric experiments based on Hamilton Markov Chain[14] computation of the expression data of hax 1 and HS1; In the last chapter, the model is described in the normal logic formula with both probabilistic condition model[15] and the results are analysed with both Hamiltonian, realization size, convergence, the rewards computation taking the CTMC as Poisson process[15] and the reachability computation with the transfer kernel of switching diffusion[16] through DTMC. In the appendix, there also includes the complete proof of model with action $\mathrm{S}$ based on Hamilton not only based on the explicit equation in this paper. Some descriptive statistics and pre-computation based on the data can be accessed through link in availability. As the process related to motor coordination and func-tion, the Hax's function in regulation, B cell's signal transduction can be further studied with more data considering its excitability and metastability functions with stimulation of drugs for instance in the future as well. And one computation applying DTMC with linear regression on previous work is made as the further extension of the model.

On one hand, as transformation on spectrum is of same computation scale level, for instance, the FFT on exponential computation, the base of normal and poisson distribution, it is natural as well to find some similar results in quantum computation. On the other hand, the property of neurons and qubits are similar, for instance all vs none (AVN) [17] and the network being heretic and some researches with regards to empiric model, either stochastically or deterministically, it is natural and beneficial to conduct research combing classical and quantomechanical computation and modelling. 


\section{Proposed Model}

Molecular interactions are studied on phenotypic data of the mRNA and its associated protein, especially the trajectory of the production of hax 1 and HS1 with interaction with each other through least action method combining diffusion process[18] in the first part while adopted model with neurons,... in the second in this paper. Furthermore, in solving the equation, one stochastic differentiation equation approximates the analytic solution and calculation of MST[19] based on converging with Hamiltonian quantities, finding three convergence points through eigenvalue of position quantities as well as satisfying $\mathrm{H}=0$ and $\mathrm{Hq}=0$ where $\mathrm{q}(\mathrm{P} X$; PY ) are momentum quantities. In the 3rd subsection, the transition is illustrated with belief graph first and then convert ratio are utilized in computing the discrete embedding of the continuous temporal logic. As comparison, the third subsection compute the discretized time markov chain as the approximation considering it as a hybrid systems.

\subsection{Switching Model with Least Action}

\section{a. population defined as mRNA and proteins}

First of all, we consider the dynamics of population of the interaction involved systems as diffusion[18], and thus the Hamiltonian $\mathrm{H}(\mathrm{x}, \theta)$ is computed with the minimization of action (quasi-potential)[20] instead of some other methods, for instance WKB[14]. With the Lagrangian denoted with respect to Hamiltonian according to LDT:

$L(x, y)=\sup \theta \in R_{n}(<y, \theta>-H(x, \theta))=<y, \theta(x, y)>-H(x, \theta(x, y))$

Due to the maximizer $\theta(\mathrm{x}, \mathrm{y})$ being implicitly defined by $\mathrm{H}_{\theta}(\mathrm{x}, \theta(\mathrm{x}, \mathrm{y}))=\mathrm{y}$, we calculate the action from quasi-potential:

$V(x 1, x 2)=\inf f_{T \geqslant 0}$ inf $\psi \in C_{x 1(0, T)}^{x 2} S T(\psi)=\inf { }_{T \geqslant 0}$ inf $\varphi \in C_{x 1(0,1)}^{x 2}$ inf $\psi \in C_{x 1(0, T)}^{x 2} S T(\psi)=\inf \varphi \in C_{x 1(0,1)}^{x 2} S(\varphi)$

So that for any $\varphi \in \mathrm{C}(0,1)$ the action $\mathrm{S}(\varphi)$ is given by the equivalent four formula:

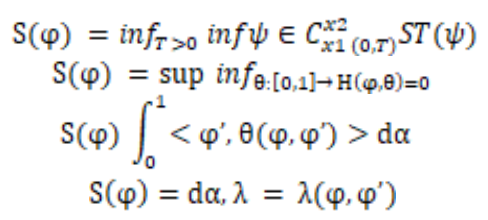

Note that $\mathrm{L}(\mathrm{x}, \mathrm{y})$ is the Lagrangian associated with the Hamiltonian $\mathrm{H}(\mathrm{x}, \theta)$ with function $\theta(\mathrm{x}, \mathrm{y})$ and $\lambda(\mathrm{x}, \mathrm{y})$ are implicitly defined for all $\mathrm{x} \in \mathrm{D}$ and $\mathrm{y} \in \mathrm{R} \mathrm{n} / 0$ as the unique solution (solution $(\theta, \lambda)$ $\in \mathrm{Rn} \times[0$,inf) of the system possessing zero value when $\varphi 0=0$ or $\lambda(\varphi, \varphi 0)=0$ setting the integrands to zero with: $\mathrm{H}(\mathrm{x}, \theta)=0, \mathrm{H} \theta(\mathrm{x}, \theta)=\lambda \mathrm{y} \leq$ lambda where the lower bounds for $\mathrm{S}(\varphi)$ is directly achieved:

$\left.\left.\mathrm{S}(\varphi)=\inf f_{T>0} \inf \psi \in C_{x 1(0, T)}^{x 2} S_{T}(\psi)^{\geq} \int_{0}^{1} \sup i n f_{\mathrm{H}(\varphi, \theta)=0}^{\theta:[0,1]}\left\langle\varphi^{\prime}, \theta\right)\right\rangle \mathrm{d} \alpha \geq \int_{0}^{1}\left\langle\varphi^{\prime}, \theta\left(\varphi, \varphi^{\prime}\right)\right)\right\rangle \mathrm{d} \alpha$

utilizing the first equation of the four. Furthermore, $S(\varphi)$ 's upper bound can also be obtained through defining a minimizing sequences $(\mathrm{T} \mathrm{k}, \psi \mathrm{k}), \mathrm{k} \in \mathrm{N}$ with the following rescaling process: For every $\mathrm{k} \in \mathrm{N}$ let: $\lambda \mathrm{k}(\alpha)=\max (\lambda(\varphi(\alpha), \varphi 0(\alpha)), 1 / \mathrm{k}), \alpha \in[0,1], \mathrm{B} \mathrm{k}(\alpha)=\mathrm{d} \alpha, \alpha \in[0,1], \mathrm{T} \mathrm{k}(\alpha)=\mathrm{B} \mathrm{k}$ $(1), B k(t)=\varphi\left(B k^{\wedge}(-1)(t)\right), t \in[0, T k]$ Specifically, the inverse of $B k$ is approximated with the Brownian standard $\sigma \mathrm{x}$ satisfying the $\alpha 0(\mathrm{t})=\lambda \mathrm{k}(\alpha(\mathrm{t}))$ and thus $1 / \mathrm{k} \leq \alpha^{6}(\mathrm{t}) \leq|\lambda \mathrm{k}|$ inf $\leq$ inf holds for all $\mathrm{t} \in[0, \mathrm{Tk}]$ with the absolute continuity of $\alpha(\mathrm{t})$. And thus, the $\psi \mathrm{k}$ is continuous in the whole 
time sequence $(0, \mathrm{~T} \mathrm{k})$, enabling the inverse process: $\mathrm{t}=\mathrm{t}(\alpha)=\mathrm{G} \mathrm{k}(\alpha)$ with $\mathrm{dt}=\mathrm{d} \alpha / \lambda \mathrm{k}$ and $\varphi^{6}$ $(\alpha)=\psi^{\prime} \mathrm{k}(\mathrm{t}) \mathrm{G}^{\prime} \mathrm{k}(\alpha)=\psi \mathrm{k}(\mathrm{t})^{\prime} / \lambda \mathrm{k}(\alpha)$.

Thus, $S_{T}(\psi)=\int{ }_{0}^{T_{k}} L\left(\varphi, \varphi \lambda_{k}^{r}\right) / \lambda_{k} d \alpha$

leading to the upper bound switching the integrate and limitation with $\mathrm{k}^{-}>$inf, and with the proof in appendix $\mathrm{B}$ (in another work with landscape model) fufilling the first order and second order conditions: $\varphi^{\prime}=\mathrm{H} \theta(\varphi, \theta) / \lambda$ is negative definite during the $\theta$ maximizing process: $\mathrm{L}(\varphi, \lambda \varphi) / \lambda$ $=\sup \theta \in \operatorname{Rn}\left(<\varphi^{\prime}, \theta>-\mathrm{H}(\varphi, \theta) / \lambda\right)$ and guaranteeing them both fufiled by $\theta=\theta\left(\varphi, \varphi^{\prime}\right)$ with the second equation, so that upper bound here is the same as the integrands of the lower bound as well as holds the $\theta=0$ when the $\lambda=0$ is satisfied, and therefore:

$$
\begin{gathered}
\frac{L\left(\varphi, \varphi \lambda_{k}^{\prime}\right)}{\lambda}=\left\langle\varphi^{\prime}, \theta \geq-\frac{H(\varphi, \theta)}{\lambda}=\left\langle\varphi_{0}, \theta\right\rangle\right. \\
\theta=\theta\left(\varphi, \varphi^{\prime}\right)
\end{gathered}
$$

The calculation can be found completely in Appendix B.

\section{b. population defined as exhibitory and inhibitory neurons}

Similarly, the switching model combining two phenomenological models with regards to the membrane potential $\mathrm{Si}$ for each cell $\mathrm{i}$ and the intrinsic oscillation characterized by phase $\varphi \mathrm{i}$ is constructed (in each single unit, either positive network or negative network is formed by the transition between pyramidal neurons and interneurons [21]):

Initial from the resting state, the periodic motion consists of the intrinsic oscillation $\mathrm{dS} / \mathrm{dt}$ with regards to cos $\varphi \mathrm{i}$ of the membrane potential $\mathrm{Si}$ and the evolution of the phase $\mathrm{d} \varphi / \mathrm{dt}$, depending on the depolarization level:

$$
\left\{\begin{array}{c}
\frac{d S i}{d t}=-S i+\sum_{j=1}^{N} W_{i j} R\left(S_{j}\right)+\mathrm{C}\left(\varphi_{i}\right)+I_{i} \\
\frac{d \varphi_{i}}{d . t}=-w+\left(\beta-\mathrm{K}\left(S_{i}\right)\right) \sin \varphi_{i}
\end{array}\right.
$$

Where $W_{i j}$ is the synaptic weight between cells $\mathrm{i}$ and $\mathrm{j}, \mathrm{R}\left(S_{j}\right)$ is the spike density of the cell $\mathrm{j}$ with sigmoid format:

$$
\begin{aligned}
R\left(S_{j}\right)=\frac{1}{2}\left(\tanh \left(g\left(S_{j}-0.5\right)\right)+1\right) & \\
& =\frac{1}{2}\left(\frac{\exp \left(g\left(S_{j}-0.5\right)\right)-\exp \left(-g\left(S_{j}-0.5\right)\right)}{\left(\exp \left(g\left(S_{j}-0.5\right)\right)+\exp \left(-g\left(S_{j}-0.5\right)\right)\right.}+1\right)
\end{aligned}
$$

And $I_{i}$ is the driving stimulus, selectively activate cell $\mathrm{i}$. Meanwhile, $\mathrm{w}$ and $\beta$ are frequency and stabilization coefficient, respectively. The couplings equations of $\mathrm{C}$ and $\mathrm{K}$ are:

$$
\left\{\begin{array}{c}
\mathrm{C}\left(\varphi_{i}\right)=\sigma\left(\cos \varphi_{i}-\cos \varphi_{0}\right) \\
\mathrm{K}\left(S_{i}\right)=\rho S_{i}
\end{array}\right.
$$


with $\rho$ and $\sigma$ modulates the coupling and initialize all cells with silent condition $(\mathrm{Si}=0)$ and equilibrium phase $\varphi_{0}=\arcsin (-\mathrm{w} / \beta)$ with parameters: $\mathrm{w}=1, \beta=1.19$ and $\mathrm{g}=10$ equals to 0.997903. Furthermore, $\cos \varphi_{0}=.542066$, the EEG is at $6 \mathrm{~Hz}$ theta oscillation computed with step changes from 0.01 to 0.1 in simulation, computational time accordingly changes from $0.01 / 6 / 2$ pi $\sim 0.000265$ to 0.002653

Specifically, in single unit:

$$
\left\{\begin{array}{c}
\frac{d S}{d t}=-S+\sigma\left(\cos \varphi-\cos \varphi_{0}\right)+I \\
\frac{d \varphi_{i}}{d t}=-w+(\beta-\rho S) \sin \varphi
\end{array}\right.
$$

Note that the fixed point attractors are considered only in this paper (process with bifurcation is not considered) since we only compute the direction from excitory population to inhibitory population while crossing the task networks without stimulus, i.e. TPN-TNN interactions. And $\rho$ is fixed at 1 while only $\sigma$ is tuned from 0 to 1 with $\mathrm{k} 5$ (6 levels in total) in this paper. In addition to the differential equations related to $\mathrm{S}$ and $\varphi$, the Weight matrix Wij abbreviated as $\mathrm{W}$ can also be defined accordingly and lead to the specific I for each $\mathrm{j}$ in neuron i prominently:

$$
W\left(\theta_{i}-\theta_{j}\right)=J^{-}+\left(J^{+}-J^{-}\right) \exp \left(-\frac{\left(\theta_{i}-\theta_{j}\right)^{2}}{2 \sigma}\right)
$$

Where $\frac{1}{360} \int_{0}^{360} W\left(\theta_{i}-\theta_{j}\right) d \theta_{j}=1, \mathrm{~J}-, \mathrm{J}+$ is the synaptic strength experimentally and theoretically respectively, leads to the Iext: $I^{\operatorname{ext}(\theta, t)=I_{0}+I_{\text {cue }}\left(\frac{1+\cos \left(\theta-\theta_{0}\right)}{2}\right)^{p}}$ at $\mathrm{p}=2, \mathrm{I}_{0}=0$ and further I as:

$$
I(\theta, t)=I^{\mathrm{ext}}(\theta, t)+\int_{-\pi}^{\pi} \frac{d \theta^{\prime}}{2 \pi} W\left(\theta-\theta^{\prime}\right) r\left(\theta^{\prime}, t\right)
$$

Where the parameter $\theta$ as the direction of each neuron $i, \theta j=2$ pi/NI is evenly distributed covering 2pi, describing each node in hopfield network (fully connected). Note that the order is fixed and can be changed iff. Wij are the same for all neurons and the detailed classical computation with normal differential equations is shown in next section while the prominent quantum computation can be found in chapter 2.3(b) and 3 .

\subsection{Approximation with numerical methods on the convert ratio}

\section{a. Gene diffusion referencing bacteria sensoring and MTS on difference mapping}

As to study the switching model interpreting the process explicitly, we thus combine the deterministic[22] background of the switching between on and off and give out one stochastic model based on the explicit (ordinary differential equation) ODE of the numbers of mRNA and proteins. Although the final model( referencing the quorum sensing model of bacteria in changing environment[23]) removes the dimers but it is used in the first place while cancelled out the in the quasi steady state according to its far more faster production and degradation rate comparing to transcription and translation.(Simplified mechanism sees Figure 1). Start from the bistability of the metastability[24] of the two state model, with the absorbing boundary conditions, $\rho 0(\mathrm{x} *, \mathrm{t})=0$ and the identification of mean transition rate with principal eigen value $\lambda 0 \varepsilon$, the quasi-stationary approximation of 


$$
\rho_{\mathrm{n}}(\mathrm{x}, \mathrm{t})=\mathrm{C} 0 * \exp \left(-\lambda^{e} t\right) \varphi \varepsilon^{0}(x, n)
$$

Furthermore, with the quasi-potential satisfying:

$$
\begin{gathered}
\Sigma_{\mathrm{neO}, 1} \operatorname{Sn}(\mathrm{x})\left(\mathrm{An}, \mathrm{m}(\mathrm{x})+\varphi^{\prime} 0(\mathrm{x}) \delta \mathrm{n}, \mathrm{m}\right) \mathrm{Fm}(\mathrm{x})=0, \\
\mathrm{H}=0.5\left(\mathrm{~g}_{\mathrm{x}} 2 \mathrm{p}_{\mathrm{x}} 2+\mathrm{g}_{\mathrm{x}} 2 \mathrm{p}_{\mathrm{x}} 2\right)+\mathrm{p}_{1} \varphi_{1}+\mathrm{p}_{2} \varphi_{2}
\end{gathered}
$$

where $\mathrm{Pi}$ is the momentum conjugate to the generalized coordinate $\mathrm{xi}$, where gi= $\sqrt{ }(\mathrm{S} 22 \mathrm{fi}+\mathrm{Xi} / \mathrm{i})$ (For more specific study of the $\varphi 1$ and $\varphi 2$ as the interacted diffusive speed, most studies applies WKB equations.) Since we focus on the transform between two status of the two populations, mRNA (of HS-1) Xn and proteins Hax1 Yn as the system.(with dimer Z of production rate $\mathrm{kXY}$ and degradation rate $\mathrm{k} P$ ) and the degradation rate of HS1 and hax1, as KX and KY, separately. From the original ODES[25]:

$$
\begin{aligned}
& \frac{\mathrm{dZ}}{\mathrm{dt}}=\mathrm{k}_{\mathrm{XY}} \mathrm{XY}-\mathrm{k}_{\mathrm{z}} \mathrm{Z} \\
& \frac{\mathrm{dX}}{\mathrm{dt}}=-\mathrm{k}_{\mathrm{XY}} \mathrm{XY}+\mathrm{k}_{\mathrm{z}} \mathrm{Z}=\mathrm{k}_{\mathrm{X}} \mathrm{X}+\frac{\mathrm{V}_{\mathrm{z}} * Z}{\mathrm{k}_{\mathrm{X}}+\mathrm{Z}} \\
& \frac{\mathrm{dY}}{\mathrm{dt}}=-\mathrm{k}_{\mathrm{XY}} \mathrm{XY}+\mathrm{k}_{\mathrm{z}} \mathrm{Z}=\mathrm{k}_{\mathrm{X}} \mathrm{X}+\frac{\mathrm{V}_{\mathrm{z}} * Z}{\mathrm{k}_{\mathrm{X}}+\mathrm{Z}}+\frac{\mathrm{V}_{\mathrm{Y}} * Z}{\mathrm{k}_{\mathrm{X}}+\mathrm{Z}}+X 0
\end{aligned}
$$

where $\mathrm{X} 0$ and $\mathrm{Y} 0$ are the initial volumes or baseline volumes of these two populations and with instant volume as VX and VY and due to the zero value of $\mathrm{dP} / \mathrm{dt}$, the term of $\mathrm{P}$ can be replaced through:

$$
\begin{aligned}
& P=-\frac{\mathrm{K}_{\mathrm{XY}}}{\mathrm{Kp}} * \mathrm{XY} \\
& \left.\frac{d X}{d t}=-\frac{\mathrm{K}_{\mathrm{X}} X}{1}+\frac{\mathrm{V}_{\mathrm{X}}}{1+\mathrm{K}_{\mathrm{X}} \mathrm{K}_{\mathrm{P} / \mathrm{K}_{\mathrm{XY}}}}+\mathrm{X} 0\right) \\
& \left.\frac{d Y}{d t}=-\frac{\mathrm{K}_{\mathrm{Y}} Y}{1}+\frac{\mathrm{V}_{\mathrm{Y}}}{1+\mathrm{K}_{\mathrm{Y}} \mathrm{K}_{\mathrm{P} /} \mathrm{K}_{\mathrm{XY}}}+\mathrm{P} 0\right)
\end{aligned}
$$

Considering the transform of $X$ (Upstream only), in the first step as degradation as the first term of right of the upper formula, the degradation part of $\mathrm{X}$ with $\mathrm{k} X$ which can be interpreted as the Poisson process and rewrite into $-\mu 1 / \exp (\mathrm{Px})$, and in the second term, the coefficient of degradation part of $\mathrm{X}, \mathrm{C} 1$ is denoted as $\mathrm{VX} * \mathrm{KX} / \mu 1$. Mean while with the assumption of continuous Markov chain, where the convert ratio of $\mathrm{Y}$ is $\mathrm{n}$, the $\mathrm{kXY} * \mathrm{X} * \mathrm{Y}$ is equivalent to $(\mathrm{Y} /(\mathrm{X}+\mathrm{Y}))^{\wedge} \mathrm{n}$ so that the whole degradation part becomes $\mathrm{C} 1 \mu 1 /\left(1+(\mathrm{y} /(\mathrm{x}+\mathrm{y}))^{\wedge} \mathrm{n}\right) \exp (\mathrm{Px})$, $\mathrm{C} 2 \mu 2 /\left(1+(\mathrm{x} /(\mathrm{x}+\mathrm{y}))^{\wedge} \mathrm{n}\right) \exp (\mathrm{Py})$, and the final transform rate of mRNA number $\mathrm{X}$ and proteins $\mathrm{Y}$ are: $\mathrm{C} 1 /\left(1+\left(\mathrm{y} /(\mathrm{x}+\mathrm{y})^{\wedge} \mathrm{n}\right)(\exp (\mathrm{Px})-1)-\mu 1 \mathrm{X}(\exp (-\mathrm{Px})-1) \operatorname{andC} 2 /(1+(\mathrm{x} /(\mathrm{x}+\mathrm{y}) \wedge \mathrm{n}(\exp (\mathrm{PY})-1)-\right.$ $\mu 2 \mathrm{Y}(\exp (-\mathrm{PY})-1)$, where the coefficient of degradation part of $\mathrm{YC} 2$ denoted as $\mathrm{Vx} * \mathrm{KY} / \mu 2$ as the reciprocal of the other population ratio. And as $\mathrm{Y}$ stands for the number of the proteins, $\mathrm{X}$ for the number of the mRNA separately with $m$ and $n$ as their translation and transcription rate. With the total sum of the system molxcule numbers assumed as $\mathrm{X}+\mathrm{Y}$, we have the Hamiltonian:C1/(1+(y/(x+y)^n)(exp(Px) - 1) - $\mu 1 \mathrm{X}(\exp (-\mathrm{Px})-1)+\mathrm{C} 2 /(1+(\mathrm{x} /(\mathrm{x}+\mathrm{y}) \wedge \mathrm{n}(\exp (\mathrm{PY}$ )$-1)-\mu 2 \mathrm{Y}(\exp (-\mathrm{PY})-1)$, where $\mathrm{Px}, \mathrm{PY}$ are calculated setting $\mathrm{H}=0$ and $\mathrm{H} \theta=0$, and convertion rate which can be calculated as $d y / d x$, specifically here, letting the first term equals the second and third equals the fourth term. (Complete see Appendix B) Note that: each single DNA population (hax 1 and HS 1) has its own degradation rate when considering about its mRNA computation and the other population's protein is taken as the intake, promoting its population when as normals binding onto the according site of persisters, activating it. Vice versa, thus, the two populations have similar structured formula describing each degradation and population under the dual interacted population. The mean switching time is calculated based on the solution of the SDE: $\mathrm{z}^{\prime}=\mathrm{z}+\sqrt{ }(\mathrm{Nc} / \mathrm{N}) \sqrt{ } 1+2 * \varepsilon-\mathrm{z} 2 * \eta$, where $\mathrm{Nc}=1 / \tau$ and $\eta \mathrm{N}(0, \delta)$ is the white noise with correlator $<\eta(\tau) \eta(\tau 0)>=\delta(\tau-\tau 0)$. Note that it is the span of the master equation in powers of 
the inverse population size $\mathrm{N}-1$ re-scaling with $\tau=2^{\wedge}(\mathrm{t} / \mathrm{N})$, and $\mathrm{z}=\mathrm{x} 1-\mathrm{x} 2$ ranges over the interval $[-1,1][20]$, leading to the solution $\tau 0 *=2 \lambda /(1-2 \lambda) \cot (\pi)$. Thus, we have the algorithm:

*********************************************************************************

Input:maximum time scale size G, mENA numbers $y$, proteins $x$, maxium steps Steps, tolerance Tol, parrameters of the sensing model (coefficient of convertion $\mathrm{c} 1, \mathrm{c} 2$, transcription and translation rate $\mathrm{m}, \mathrm{n}$, degradation rate $\mathrm{k} 1, \mathrm{k} 2$, formation coefficients mu1, mu2, diffusion rate $\mathrm{b} 1, \mathrm{~b} 2$ ), $\mathrm{dt}$ as time increasement Initilize: maximum time scale, T, maximum step number steps, tolerance Tol, numbers of mRNA after the first diffusion process that if necessary, initialized as one random the number, in the first status we start with the largest interval to cover higher possibilities, i.e. $[\mathrm{x}(0), \mathrm{x}(0)+1, \ldots, \mathrm{x}[1]-1]$,

for do

xhat (length(xhat)) $<\mathrm{x}(\mathrm{i}+1)$; repeat

record the sizeT, time $t$, steps, Steps - steps +1

set the sequence according to size $\mathrm{T}$ (the interval for mRNA numers) $\mathrm{x}(1), \mathrm{x}(2), \ldots, \mathrm{x}(\mathrm{T})$ and generate the population numer of proteins according data distriutio, $y(1), y(2) \ldots y(T)$. T initialized as the $X(i+1)-$ X(i), consider Hamiltonion Markov (Hierarchical)[26]:

if xhat exist(iterated from previous status) then:

segment the interval into several sub-sequences(X0 as the new current status, $\mathrm{X} 1$ as the previous status)

end if

Note that: As we only consider up streaming, down regulation into those efore the previous status is not included.

Function dynamics inputs: $\mathrm{x}$ and fined $\mathrm{y}$ (or $\mathrm{x} 0$, y0, or $\mathrm{x} 1, \mathrm{y} 1$ )

Calculate degradation term $\mathrm{w} 1$ and $\mathrm{w} 2$ according to the $(* 1)$

Calculate px, py, dx, dy, conversion rate, $\mathrm{He}$ and $\mathrm{Hx}, \mathrm{s}$ according to Appendix 2 predict multiplied mRNA and protein numbers xhat, yhat, and other Hamiltonians

Calculate update gamma, delta

Calculate tolerance for further stopping criteria as the residule of gamma and cell number with:

$$
\begin{gathered}
\text { tol }=\text { abs }(\text { Gamma-gamma }) / \text { gamma; } \\
\text { toll }=\text { mean }(\operatorname{abs}(\text { xhat }-x 1) / x 1+\text { abs }(\text { yhat }-y 1) / y 1)
\end{gathered}
$$

Output: H, XHat, HthetaX, HthetaY, HxX, HxY, HamilX, HamilY, HamulXhat, HamilYhat, xhat, sX, sY, px, py, pxhat, pyhat, actionratio, delta, gamma, Delta, Gamma, cr1, c1, c2, crhat, c1hat, c2hat, tol,toll, Txc, Tyc, Txchat, Tychat

Concatenate results:

If $\mathrm{X} 0, \mathrm{X} 1$ exist then:

$$
\text { Xhat }=[\text { X0hat, X1hat }]
$$

\section{End if}

If only $\mathrm{x} 0$ exist then

End if

$$
\text { Xhat }=\text { X0hat; }
$$

Do similar prediction regenerate the mRNA numbers $\mathrm{X}$ according to $\mathrm{Y}$ with

Function dynamics again for comparison. Results are with postfix ' $L$ '

Store the quantities of 'successful' moves with smaller tolerance and action for either from mRNA or protein numbers.

If satisfies the configuration condition then

\section{Else}

$$
\text { Tol }=\min (\text { tol }+ \text { toll, toll }+ \text { toll }) \text {; }
$$

End if

$$
\text { Fail++ }
$$




\section{b. Neuron switching in single unit based on normal differential equation}

Derived from the equation $(* *)$ of single unit fixed point attractors, the normal form around bifurcation point can be computed with:

$$
\left\{\begin{array}{c}
\frac{d S}{d t}=a S^{2}+\lambda_{1} S \\
\frac{d \varphi}{d t}=\lambda_{2} \varphi
\end{array}\right.
$$

In addition to the computation of $\lambda$ demonstrated in chapter 3 and the regression of the parameters q, based on experiment data mentioned in 2.1, the global feedback inhibition from the excitatory neurons $\mathrm{j}$ to the single unit $\mathrm{i}$ is depicted as the time series with regards to frequency domain as well through firing rate model and leaky-integrating differential equation:

$$
\begin{gathered}
I_{i}=\tau_{i} \frac{d I_{i}}{d t}+\sum_{j=1}^{N I} w_{i j} R\left(I_{j}\right)-G\left[\sum_{j=1}^{N I} R\left(I_{j}\right)\right]+I_{\text {ext }} \\
\text { Where } R\left(I_{j}\right)= \begin{cases}\ln \left(\frac{I j}{I_{\text {thre }}}\right), & \text { if } I_{j}>I_{\text {thre }} \\
0, & \text { otherwise }\end{cases} \\
G\left[R\left(I_{j}\right)\right]=\left\{\begin{array}{cc}
\sum_{j=1}^{N I} \ln \left(\frac{I j}{I_{\text {thre }}}\right) / N I, & \text { if } I_{j}>I_{\text {thre }} \\
0, & \text { otherwise }
\end{array}\right.
\end{gathered}
$$

Note that, to simplify the computation, among the parameters conductance, eigenvalue of the Jacobian of fixed points and synaptic strength, only the conductance is changed. And the algorithm shows the TNN single unit only here:

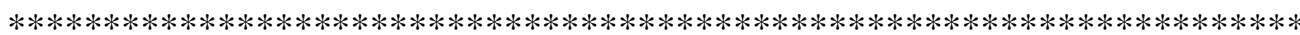

Input: $\mathrm{k} 5$ and prominent conductance for excitatory NMDA gNMDAE, conductance for inhibitory NMDA gNMDAI, conductance for excitatory AMPA gAMPAE, conductance for inhibitory AMPA g AMPAI, conductance for excitatory GABA gGABAE, conductance for inhibitory GABA gGABAI, NI, Iext, time step $\mathrm{dt}=\mathrm{tao} / 1000$, rest membrane potential $\mathrm{Vm}$, capacitance $\mathrm{Cm}$, leak conductance $\mathrm{gL}$, leak voltage VL, resistance sigma, experimental synaptic strength $\mathrm{J}$-, weight matrix $\mathrm{W}$, neuron direction theta Initial: $\mathrm{t}=0, \mathrm{dV} \_\mathrm{dt}=0$ and $\mathrm{I} 1=$ Ithre, in the first status we start from excitatory NMDA(nonselective) to inhibitory GABA populations considering Iext from the excitatory AMPA(selective) and thus we have the $\mathrm{I}_{\mathrm{NMDA}}$ controlled by $\mathrm{Mg} 2+$ and $\mathrm{V}_{\mathrm{E}}=0: \mathrm{I}_{\mathrm{NMDA}}=\operatorname{sum}\left(\mathrm{Vm} / \mathrm{NI} / \operatorname{sum}\left(1+\mathrm{c} \_\mathrm{MG} * \mathrm{np} \cdot \exp (-0.062 * \mathrm{Vm} / \mathrm{NI} / 3.57)\right)\right)$, Iext $=\operatorname{sum}\left(\exp (\mathrm{I} 0) / \operatorname{sqrt}\left(2 \mathrm{pi}^{*} \operatorname{sigma}\right)^{*} \exp \left(-(\text { theta-theta } 0)^{\wedge} 2 / \operatorname{sigma}\right)\right.$, where theta $0=0$ and theta $=[0,2 \mathrm{pi} / \mathrm{Ni}$, $\ldots, 2 \mathrm{pi}]$

\section{I.achieve V(t)}

for do $t=0, d t, \ldots$, tao

1. compute the increase of $\mathrm{V}$ each time step: $\mathrm{dV} \_\mathrm{dt}=(-\mathrm{gL} *(\mathrm{Vm}-\mathrm{VL})-\mathrm{I0}) / \mathrm{Cm}$

end

2. update $\mathrm{V}(\mathrm{t}+\mathrm{dt})=\mathrm{V}(\mathrm{t})+\mathrm{dV} \_\mathrm{dt}=\mathrm{V}(\mathrm{t})+(-\mathrm{gL} *(\mathrm{Vm}-\mathrm{VL})-\mathrm{I} 0) / \mathrm{Cm}$

II.achieve Excitatory term Ex and Inhibitory term In and compute simulated I

for do $t=0, d t, \ldots$, tao

1. compute increase of $\mathbf{I}$ across all cell $\mathbf{j}$ to $u$ unit $\mathbf{i}$ at each time step: $\mathrm{dIi}$ - $\mathrm{dt}=\mathrm{I}_{\mathrm{NMDA}}$ for do $\mathrm{j}=1,2, \ldots .4096$

1. Compute from excitatory status:

$$
\mathrm{I}_{\mathrm{AMPA} \_\mathrm{E}, \mathrm{j}}=\left(\mathrm{V}(\mathrm{t})-\mathrm{V}_{\mathrm{E}}\right)^{*} \mathrm{gAMPAE} * \frac{\left(1+\cos \left(\text { thet }_{j}-\text { thet }_{0}\right)\right)^{2}}{4}
$$


2. Compute from inhibitory status:

$$
\begin{aligned}
& \mathrm{I}_{\mathrm{AMPA}_{-} \mathrm{I}, \mathrm{j}}=\left(\mathrm{V}(\mathrm{t})-\mathrm{V}_{\mathrm{E}}\right) * \mathrm{gAMPAI} * \frac{\left(1+\cos \left(\text { thet }_{j}-\text { thet }_{0}\right)\right)^{2}}{4} \\
& \mathrm{I}_{\mathrm{GABA}_{-} \mathrm{I}, \mathrm{j}}=\left(\mathrm{V}(\mathrm{t})-\mathrm{V}_{\mathrm{E}}\right) * \mathrm{gGABAI}^{*} \frac{\left(1+\cos \left(\text { thet }_{j}-\text { thet }_{0}\right)\right)^{2}}{4}
\end{aligned}
$$

3. Transfer I to firing rate $\mathrm{R}$ (according to equation $\mathrm{a}$ ):

$\mathrm{R}_{\mathrm{AMPA} \_E, \mathrm{j}}\left(\mathrm{I}_{\mathrm{AMPA} \_ \text {E, j }}\right), \mathrm{R}_{\mathrm{AMPA} \_\mathrm{I}, \mathrm{j}}\left(\mathrm{I}_{\mathrm{AMPA} \_\mathrm{I}, \mathrm{j}}\right), \mathrm{R}_{\mathrm{GABA} \_\mathrm{I}, \mathrm{j}}\left(\mathrm{I}_{\mathrm{GABA} \_\mathrm{I}, \mathrm{j}}\right)$

4. With weight matrix: wij $=^{J^{+}+\left(J^{+}-J^{-}\right) \exp \left(-\frac{\left(\theta_{i}-\theta_{j}\right)^{2}}{2 \sigma}\right)}$, compute exitatory term $\mathrm{Ex}=\sum_{j=1}^{N I} w_{i j} R\left(I_{j}\right)$

$\mathrm{Ex}=\mathrm{Ex}+$ wij $^{*} \mathrm{R}_{\mathrm{AMPA} \_\mathrm{E}, \mathrm{j}}\left(\mathrm{I}_{\mathrm{AMPA} \_\mathrm{E}, \mathrm{j}}\right)$

5. Do for inhibitory I: Transfer $\mathrm{R}$ to gain $\mathrm{G}$ (according to equation $\mathrm{b}$ ):

$$
\mathrm{G}\left[\mathrm{R}_{\mathrm{AMPA} \_\mathrm{I}, \mathrm{j}}\left(\mathrm{I}_{\mathrm{AMPA} \_\mathrm{I}, \mathrm{j}}\right)\right], \mathrm{G}\left[\mathrm{R}_{\mathrm{GABA} \_\mathrm{I}, \mathrm{j}}\left(\mathrm{I}_{\mathrm{GABA} \_-} \mathrm{I}, \mathrm{j}\right)\right]
$$

6. Compute inhibitory term $\mathrm{In}=G\left[\sum_{j=1}^{N I} R\left(I_{j}\right)\right]$ :

$\mathrm{In}=\mathrm{In}+\mathrm{G}\left[\mathrm{R}_{\mathrm{AMPA} \_\mathrm{I}, \mathrm{j}}\left(\mathrm{I}_{\mathrm{AMPA} \_\mathrm{I}, \mathrm{j}}\right)\right]+\mathrm{G}\left[\mathrm{R}_{\mathrm{GABA} \_\mathrm{I}, \mathrm{j}}\left(\mathrm{I}_{\mathrm{GABA} \_\mathrm{I}, \mathrm{j}}\right)\right]$

end

7. Compute dIi_dt $=\mathrm{dI} \_\mathrm{dt}+\mathrm{I}_{\mathrm{AMPA} \_\mathrm{E}, \mathrm{j}}$

end

2. Compute the total I at time $\mathbf{t}: \operatorname{Ii}(\mathbf{t})=\tau_{i} * d I d t+\mathrm{Ex}-I n+I_{\text {ext }}$

Output: Ii(t), dI_dt, Ex, In

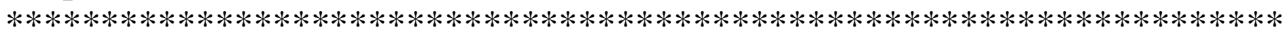

\subsection{Stochastic Model for (a) gene switches}

\subsubsection{Probailistic Uncertainty Conditional}

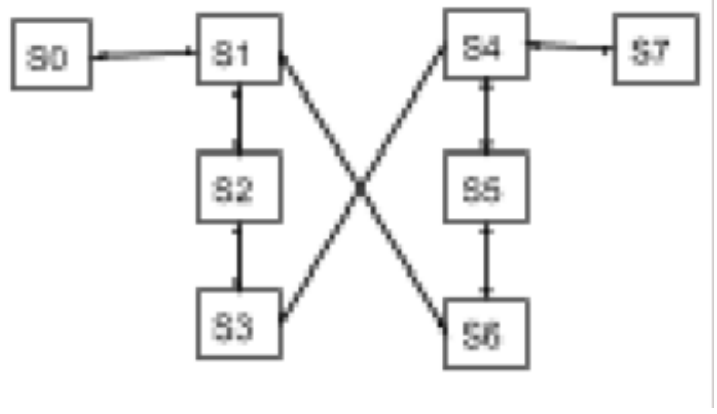

Figure1: Belief Graph

Where S0 = hax1_DNA^inactive ${ }^{\wedge}$ hax 1_DNA^low_concentration

S1 = hax1_DNA^active ${ }^{\wedge}$ hax1 DNA^high_concentration

S2 $=$ hax1_mRNA $\wedge^{\wedge} \neg$ degradate

S3 $=$ hax 1_protein ${ }^{\wedge} \neg$ degradate

S4 = HS1_DNA^inactive ${ }^{\wedge} \mathrm{HS} 1$ _DNA^low_concentration

S5 $=$ HS1_mRNA^active ${ }^{\wedge}$ HS1 DNA^^high_concentration

S6 $=$ HS1_mRNA^^degradate

S7 $=$ HS1_protein^^degradate

Transition rate[27]: $\mathrm{R} / \mathrm{P}(\mathrm{R}$ for CTMC, $\mathrm{P}$ for DTMC) 


\subsubsection{Model Check for Stochastic Models Combining Continuous Time Markov Chain with Embedding in Reward Computation}

The logic applied on a probabilistic notion regards to the belief graph is based on the trust which is reflected by the reliability and predictability. Specifically, the language of the stochastic models used for computing CTMC [28]is the Continuous Stochastic Logic(CSL) developed and extended by some research

A CTMC is a tuple $C=(S, s, R, L)$ where $S$ is the finite set of states, $s$ is the initial state ; $R$ is $\mathrm{S} * \mathrm{~S}->\mathrm{R}>0$ is the transition rate matrix; $\mathrm{L}: \mathrm{S}->2 \mathrm{AP}$ is a labelling function which assigns to each state $\mathrm{s} € \mathrm{~S}$ is the set $\mathrm{L}(\mathrm{s})$ of atomic propositions valid in the state. Instead of the case of DTMCs, a fixed set of atomic propositions AP is applied, the transition rate matrix R assigns rates to each pair of states in the CTMC, used as parameters of the exponential distribution. A transition can only occur between states $\mathrm{s}$ and $\mathrm{s}^{\prime}$ if $\mathrm{R}(\mathrm{s}, \mathrm{s})>0$, representing the probability of this transition being triggered within $\mathrm{t}$ time-units equals $1-\mathrm{e}-\mathrm{R}\left(\mathrm{s}, \mathrm{s}^{\prime}\right) \mathrm{t}$. Time spent in state $\mathrm{s}$, before such transition occurs, is exponentially distributed with rate $\mathrm{E}(\mathrm{s})$, where: $\mathrm{E}(\mathrm{s})=\operatorname{sum}\left(\mathrm{R}\left(\mathrm{s}, \mathrm{s}^{\prime}\right)\right)$, where $\mathrm{E}(\mathrm{s})$ is known as the exit rate of state $\mathrm{s}$.

The embedded DTMC of a CTMC, is the probability of each state s' transitioned from the precious s, independent of the time, defined as:

$\operatorname{Emb}(\mathrm{C})=(\mathrm{S}, \mathrm{s}, \mathrm{Pemb}(\mathrm{C}), \mathrm{L})$ where for $\mathrm{s}, \mathrm{s}^{\prime} € \mathrm{~S}$ :

$\operatorname{Pemb}(\mathrm{C})\left(\mathrm{s}, \mathrm{s}^{\prime}\right)=\left\{\left\{\begin{array}{c}\mathrm{R}\left(\mathrm{s}, \mathrm{s}^{\prime}\right) / \mathrm{E}(\mathrm{s}), \text { if } \mathrm{E}(\mathrm{s}) \neq 0 \\ 1, \text { if } \mathrm{E}(\mathrm{s})=0 \text { and } \mathrm{s}=\mathrm{s}^{\prime} \\ 1, \quad \text { otherwise }\end{array}\right.\right.$

where the behavior of the CTMC in the alternative way remains in a state s delayed and exponentially distributed with rate $\mathrm{E}(\mathrm{s})$ and transit with $\mathrm{Pemb}(\mathrm{C})\left(\mathrm{s}, \mathrm{s}^{\prime}\right)$.

The infinitesimal generator matrix for the CTMC $C=(S, s, R, L)$ is the matrix $Q$ : $S * S->R$ defined as:

$$
\mathrm{Q}\left(\mathrm{s}, \mathrm{s}^{\prime}\right)=\mathrm{R}\left(\mathrm{s}, \mathrm{s}^{\prime}\right) \text {, if } \mathrm{s} \text { is not } \mathrm{s}^{\prime}-\sum \mathrm{s}^{\prime \prime} \neq \mathrm{s}, \mathrm{R}\left(\mathrm{s}, \mathrm{s}^{\prime}\right) \text { otherwise }
$$

The CTMC stores the transition from $\mathrm{s}$ to $\mathrm{s}$ ' in ratio format instead of the possibility in DTMC.

However, the probability measures $\operatorname{Prs}$ on $\sum \operatorname{PathC}(\mathrm{s})$ as the unique measure such that $\operatorname{Prs}(\mathrm{C}(\mathrm{s}))=$ 1 and for any cylinder $\mathrm{C}\left(\mathrm{s}, \mathrm{I}, . ., \mathrm{In}-1, \mathrm{sn}, \mathrm{I}^{\prime}, \mathrm{s}^{\prime}\right), \operatorname{Prs}\left(\mathrm{C}\left(\mathrm{s}, \mathrm{I}, \ldots, \mathrm{In}-1, \mathrm{Sn}, \mathrm{I}, \mathrm{s}^{\prime}\right)\right)$ equals:

$\operatorname{Prs}\left(C\left(s, I_{, \ldots}, \operatorname{In}-1, \operatorname{sn}\right)\right)=\operatorname{Prs}(C(s, I, \ldots, I n-1, s n)) * \operatorname{P} 1 e m b(C 1)\left(s n, s^{\prime}\right)\left(e-E(s n) * \operatorname{infl}^{s}-e-E(\operatorname{sn}) * \operatorname{supl}^{\prime}\right)$

In our case, such model check as with PCTL, we can easily derive the path formulae for the states between S0 and S7 separately with 6 time intervals $\mathrm{I}=[\mathrm{t} 0, \mathrm{ti}]$ :

$\mathrm{P} \sim \mathrm{p}[0 \mathrm{I} \varphi]=\mathrm{P} \sim \mathrm{p}[$ trueUI $\varphi]$,

$\mathrm{P} \sim \mathrm{p}[0 \mathrm{I} \varphi]=\mathrm{P} \sim \mathrm{p}[$ exist UI $\varphi], \quad \varphi=$ 'transit, Stands for the probability that a transition occurs in time interval $\mathrm{I}=[\mathrm{t} 0$, ti], And thus, For determing the least solution,

ProbC, $\left(\mathrm{s}, \varphi i_{s} \mathrm{U}[0, \mathrm{t}], \psi\right)$

$$
=\int \sum \operatorname{Pemb}(\mathrm{C})\left(\mathrm{s}, \mathrm{s}^{\prime}\right) * \mathrm{E}(\mathrm{s}) * \mathrm{e}-\mathrm{E}(\mathrm{s}) * \mathrm{X} * \operatorname{ProbC}\left(\mathrm{s}^{\prime}, \varphi \mathrm{i}, \mathrm{U}[0, \mathrm{t}], \psi\right)
$$


And define the rewards function a CTMC $\mathrm{D}=(\mathrm{S}, \mathrm{s}, \mathrm{R}, \mathrm{L})$, the semantics is defined as: $\mathrm{S} \mid=\mathrm{R} \sim \mathrm{r}[\mathrm{I}=$ $\mathrm{t}], \operatorname{ExpC}(\mathrm{s}, \mathrm{XI}=\mathrm{t}) \sim \mathrm{r}$

\subsubsection{Model Check for Stochastic Models reachability/safety computing based on Discrete Time Markov Chain(DTMC) approximating the Discrete Time Markov Process(DTMP)}

In the second application of model check, the continuous dynamics described by switching diffusions is studied with reachability and dually safety properties on DTMC.Compared with the MC on continuous time domain, DTMC is defined with a fixed, finite set of atomic propositions used to label states. The DTMC D is a tuple similar as CTMC (S,s,P,L), where $\mathrm{S}$ is a finite set of states; $\mathrm{s}$ is the initial states;

$\mathrm{S} * \mathrm{~S}->[0,1]$ is the transition probability matrix where $\sum \mathrm{s} P\left(\mathrm{~s}, \mathrm{~s}^{\prime}\right)=1$ for all $\mathrm{s} € \mathrm{~S}$ where $\mathrm{L}(\mathrm{s})$ of atomic propositions are valid.

$$
(\max \{d q(t 1, t 2)\}<=K d *|t 2-t 1|,)
$$

and $\mathrm{K}>=12$ is the Dudley metric universal constant. Let $\mathrm{h}$ defined larger than 0 be a sampling time and the mean $\mathrm{E}$ and the covariance $\mathrm{C}$ to simulate a nomal distribution $\mathrm{N}(\mathrm{x} \mid \mathrm{E}, \mathrm{C})$. Then, the discreete kernel is

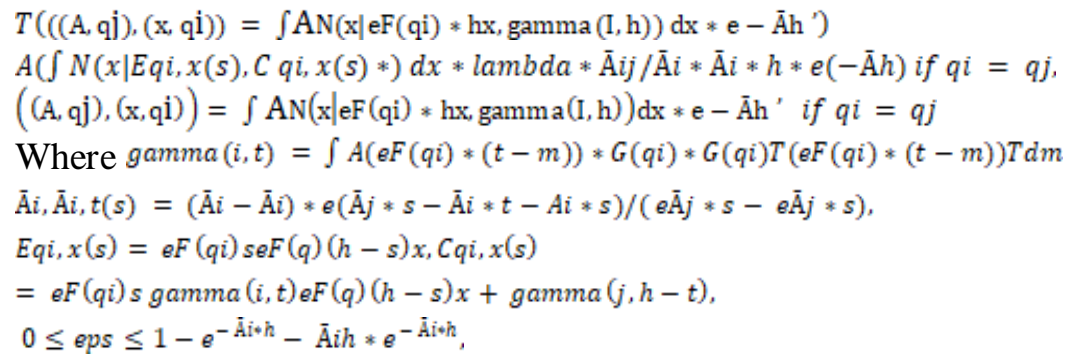

With the events on t belongs to I, An $=\{X(t) \in S \mid P \sim p[1]=,P \sim p[$ true UI $\varphi]\}, \quad B n=\{X(t) \in S \mid$ $\mathrm{P} \sim \mathrm{p}[\diamond \mathrm{I} \varphi]=\mathrm{P} \sim \mathrm{p}$ [exist UI $\varphi], \quad \operatorname{Psafe}(\mathrm{X}, \mathrm{S}, \mathrm{I})=\operatorname{limP}\left(\mathrm{An}{ }^{\wedge} \mathrm{Bc}\right), \operatorname{Preach}(\mathrm{X}, \mathrm{Sc}, \mathrm{I})=1-\operatorname{limP}\left(\mathrm{An}{ }^{\wedge} \mathrm{Bc}\right)$

$$
\begin{aligned}
\int \sum \mathrm{Tdx}(\mathrm{z} 1, \mathrm{z} 2)=\mathrm{T}(\mathrm{z} 1, \mathrm{z} 2), \text { if } \mathrm{z} 1, \mathrm{z} 2 € \mathrm{~S} \mathrm{dx} & =1-\sum \mathrm{zj} \in S d x r \\
& =-1, \quad \text { if } \mathrm{z} 1, \mathrm{z} 2 € \varphi, \quad \text { if } z 1 € S d x, z 2, z 2 € S \\
& =0, \quad \text { if } \mathrm{z} 1 € \varphi, \mathrm{z} 2 € \mathrm{~S} \mathrm{dx}
\end{aligned}
$$

Continuous kernel proof see Appendix B.

To compute the reachability/safety properties, we introduce the scheme based on Discrete Time Markov Chain(DTMC) which discretize the state space to approximate the Discrete Time Markov Process(DTMP) results from the original switching diffusion process $H$, a tuple $H=(Q$, $\mathrm{K}, \mathrm{F}, \mathrm{G}, \mathrm{W}, \wedge)$, where $\mathrm{Q}=\{\mathrm{q} 1, \ldots, \mathrm{q}|\mathrm{Q}|\}$ is the set of discrete modes instead of the matrix in CTMC and $\mathrm{Y}=(\mathrm{X}, \alpha)$ its solution. For any $\mathrm{q} \in \mathrm{Q}$, call $\mathrm{Xq}$ the solution of the SDE : $\mathrm{Xq}(\mathrm{t})=$ $\mathrm{F}(\mathrm{q}) * \mathrm{Xq}(\mathrm{t}) \mathrm{dt}+\mathrm{G}(\mathrm{q}) * \mathrm{dW}(\mathrm{t})(*)$ In this section we assure that $\mathrm{Xq}$ is a ui-dimensional, zero mean Gaussig, an process (GP). Xq is almost surely bounded within the interval I by Assumption. Set $\mathrm{h}$ $=\min \{2(-n) /(2 * \sqrt{ }(2) * K 2 * K d), 2-n\}$ and $\epsilon \mathrm{n}=2(-n / 2)$, where $n \in N$, and $K d$ is a constant such that for any $\mathrm{t} 1, \mathrm{t} 2 € \mathrm{I}$ 


\subsection{Quantum Computation Model for (b)neuron spikes}

In accordance with classical computation, we use the entanglement of two neutral atoms $\mathrm{x} 1, \mathrm{x} 2$ (fast two-qubit gates) to compute the transmission between two eigenstates here denoted as $|\mathrm{g}\rangle$ and $|\mathrm{e}\rangle$, alternatively $|0\rangle$ and $|1\rangle$. Hamiltonian holding time and position information is utilized to express the interactions similarly separated into internal and external parts:

$$
H(t, x 1, x 2)=\operatorname{Hex}(t, x 1, x 2)+\operatorname{Hin}(t, x 1, x 2)
$$

Briefly, Hext can be spanned from dipole-dipole interaction around distance $r=|x 1-x 2|$ and the photon kick[22] happened during absorption from $|\mathrm{g}\rangle$ to Stark eigenstate $|\mathrm{r}\rangle[21]$. We focus on the internal interactions mainly with phase gates with internal dynamics induced by the standard Hamiltonian:

$$
\begin{gathered}
H^{i}(t, x 1, x 2)=u\left|r>_{1}<r\right| \otimes\left|r>_{2}<r\right|+ \\
\sum_{j=1,2}\left[\left(d_{j}(t)-i \gamma\right) \mid r>_{j}<-\frac{\Omega_{j}\left(t, \mathrm{x}_{j}\right)}{2}\left(\left|g>_{j}<r\right|\right)\right]
\end{gathered}
$$

Where $\mathrm{d}(\mathrm{t})$ detunings and $\Omega_{j}\left(t, \mathrm{x}_{j}\right)$ Rabi frequencies describes the exciting lasers while $\gamma$ accounts for decay from the excited states $|\mathrm{r}\rangle$.

Next, we focus on the transfer of the system with the Hamiltonian:

$$
H=\sum_{a, b} H^{a, b} \otimes\left|a>_{1}<a\right| \otimes\left|b>_{2}<b\right|
$$

Where $H^{a, b}=\sum_{i=\{a, b]}\left[\frac{p i^{2}}{2 m}+V^{i}\left(\mathrm{x}_{i}, t\right)\right]+u^{a, b}\left(\mathrm{x}_{1}-\mathrm{x}_{2}\right)$ with pi being the momentum operator.

Each single unit of neuron model can be seen as one single state either being excitory or inhibitory analogue to the logical states $\mid 1>$ or $\mid 0>$ and the direct switching equals to the entanglement with energy shift while: 


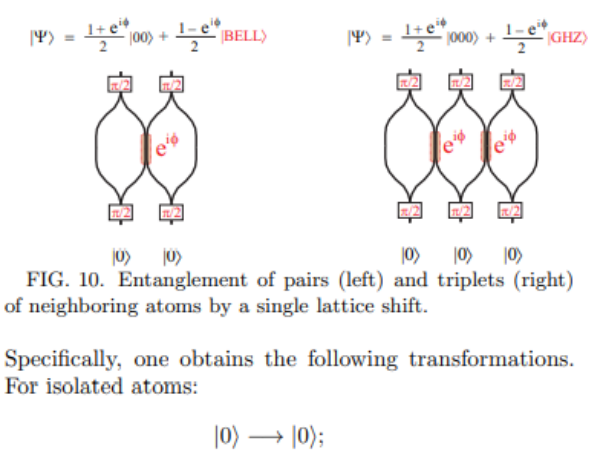

for pairs of neighboring atoms:

$$
\left.|00\rangle \longrightarrow \frac{1+e^{i \psi}}{2}|00\rangle+\frac{1-e^{i \phi}}{2} \mid \text { BELL }\right\rangle ;
$$

and for triplets of neighboring atoms:

$$
|000\rangle \longrightarrow \frac{1+e^{i \phi}}{2}|000\rangle+\frac{1-e^{i \phi}}{2}|\mathrm{GHZ}\rangle ;
$$

where we have used the notation

$$
\begin{aligned}
\mid \text { BELL }\rangle & =\frac{1}{\sqrt{2}}\{|0\rangle|+\rangle-|1\rangle|-\rangle\}, \\
\mid \text { GHZ }\rangle & =\frac{1}{\sqrt{2}}\{|0\rangle|+\rangle|1\rangle-|1\rangle|-\rangle|0\rangle\},
\end{aligned}
$$

Basically, the truth table can be achieved through the fundamental phase gate:

$|0>| 0>->|0>| 0>$

$|0>| 1>->|0>| 1>$

$|1>| 0>->|1>| 0>$

$|1>| 1>->e^{-\ln \left(-2 \varphi_{r}^{a b}+\varphi_{r}^{b b}\right)}\left|b>_{1}\right| b>_{2}$

applying a single-bit

rotation:

$$
|0><0| e^{-i \varphi_{r}^{a}}+|1><1| e^{-i\left(\varphi_{r}^{a}+\varphi_{r}^{a b}\right)}
$$

Further more, in such a scheme, a NI-bit code where the codewords:

$\mid 0 \mathrm{~s}>=((|000>+| 111>) / \sqrt{ } 2)^{3}$

$\mid 1 \mathrm{~s}>=((|000>-| 111>) / \sqrt{ } 2)^{3}$

Composed by GHZ states[23], embed the

2D qubit's Hilber space into $2^{\mathrm{NI}}$

D qubit's Hilber space:

$$
\mathcal{H} \ni \alpha|0\rangle+\beta|1\rangle \mapsto \alpha\left|0_{\mathrm{S}}\right\rangle+\beta\left|1_{\mathrm{S}}\right\rangle \in \mathcal{H}_{\mathrm{E}} \subset \mathcal{H}^{\otimes 9} .
$$

Another entanglement is to transport the state $\mid r>$ excited from either $\mid 0>$ or $\mid 1>$ and then move the lattice:

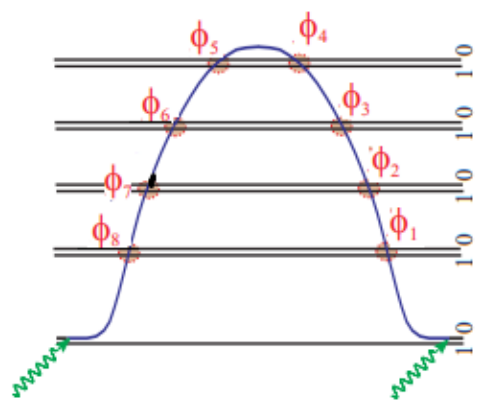

With a selelcted atom in the state $(|0>+| \mathrm{r}>)$ onto a string of NI atoms in $(|0>+| 1>){ }^{\otimes N I}$, we can see the sweep entanglement as moving the transport lattice to sweep the selected atom across the $\mathrm{N}$ lattice and transform the state of atoms onto $e^{i \varphi^{0}}\left|0>+e^{i \varphi^{1}}\right| 1>$ with $\varphi=\varphi^{1}-\varphi^{0}$ a differential phase and result to the resulting state:

$$
\begin{aligned}
& \mid 0>(|0>+| 1>) \ldots(|0>+| 1>)+ \\
& \mid r>e^{i N \varphi^{0}}\left(\left|0>+e^{i \varphi}\right| 1>\right) \ldots\left(\left|0>+e^{i \varphi}\right| 1>\right)
\end{aligned}
$$

For $\varphi=$ pi, the GHZ on N+1 dimension can be swept to

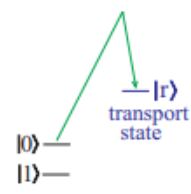
the standard form:

$$
|\psi\rangle=\frac{1}{\sqrt{2}}(|0\rangle|0\rangle|0\rangle \cdots|0\rangle+|1\rangle|1\rangle|1\rangle \cdots|1\rangle)
$$

with one operation.

\section{ApPliCAtion AND Results}

To have a clearer understanding of the switching process com-bining the binding with increasing and decreasing speed both ofhax 1 and HS 1 , the two population are regarded as promoters and resistors both when activating and deactivating each other's production.(The coarse process can be briefly described as in figure 2 , and it is briefly introduced in the previous chapter.) 


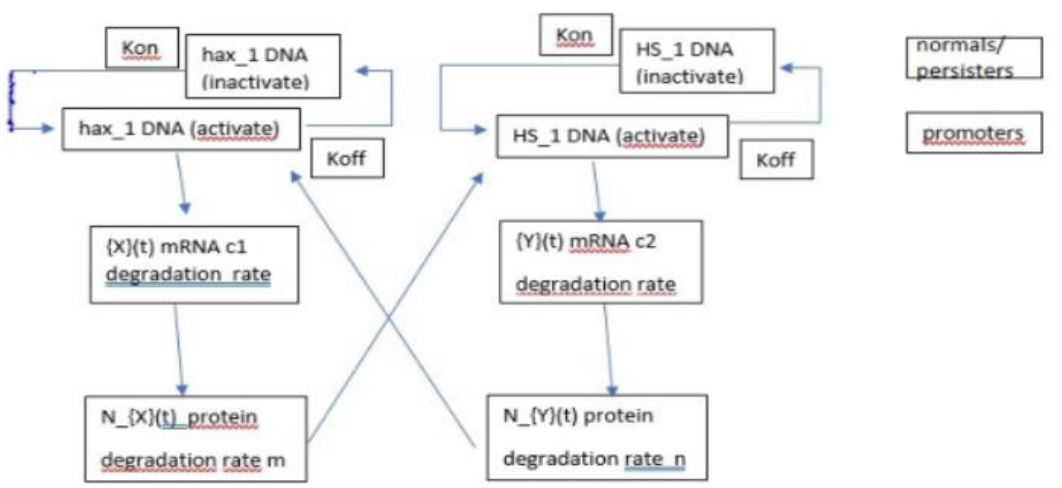

(a)

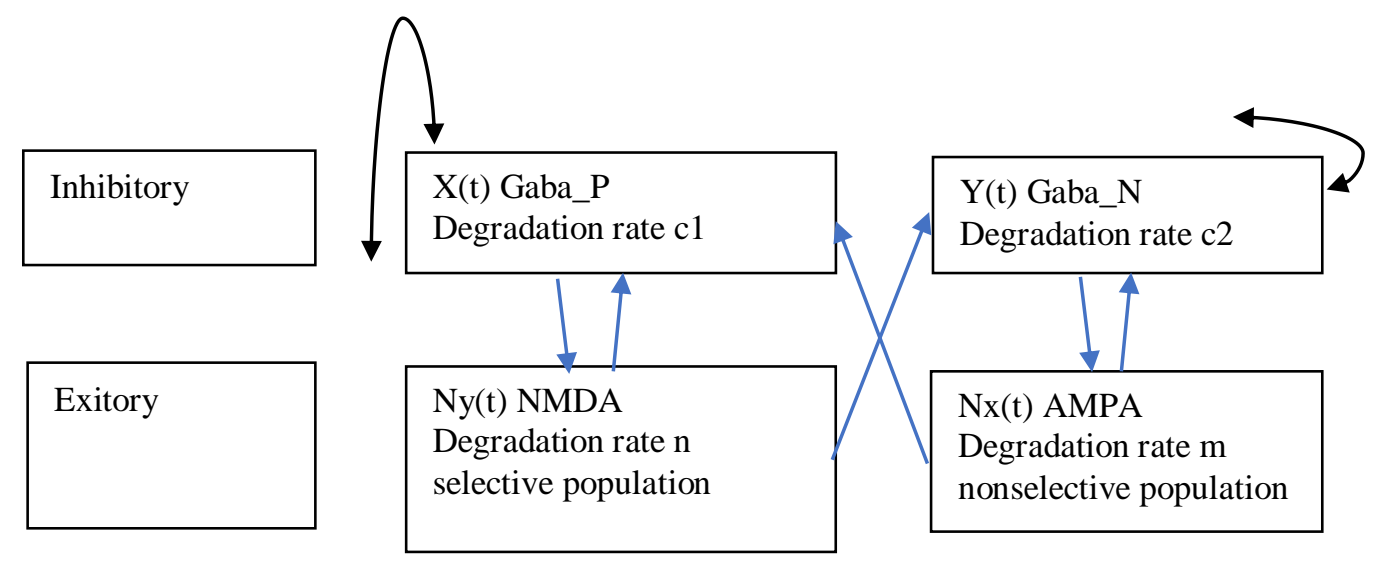

(b)

Figure 2: Pipeline for (a) top: gene model; (b) bottom: neuron model

\subsection{Stability Analysis}

\section{a. Three critical points for the gene model}

As we have data (see Appendix C) of 15 status in all both for hax1and HS 1 with their different cell numbers taken as $\mathrm{X}$ and $\mathrm{Y}$ in our model. For reward computation for their Markov chain, we pre-compute the their Hamiltonians, Action Potentials, mean switching time and related dynamics i9n the form (see availability), and the 6upstreaming status, which is the focus of the experiment application of our model. Using the pre-computation results, we are able to discuss about some practical problems about the current model. There are three groups of quantities studied combining the action potential as well as Hamiltonian inspired by bacterial quorom sensoring, 'momentum and cell numbers', 'MTS with the SDE', and 'corresponding Hamiltonians', of each transform status in Appendix A.

First, we use the taylor expansion to simplify the four ODE achieved in Appendix B: to

$$
\begin{aligned}
& \mathrm{dx}=\mathrm{C} 1 /(1+(\mathrm{y} /(\mathrm{x}+\mathrm{y}) \mathrm{mPx}-\mu 1 * \mathrm{X} * \mathrm{PX}) \\
& \mathrm{dy}=\mathrm{C} 2 /\left(1+\left(\mathrm{x} /(\mathrm{x}+\mathrm{y}) \mathrm{nP}_{\mathrm{Y}}-\mu 2 * \mathrm{y} * \mathrm{P}_{\mathrm{Y}}\right)\right. \\
& \mathrm{dPx}=\mathrm{C} 2 \mathrm{~m}\left(\mathrm{x} /(\mathrm{x}+\mathrm{y}) \mathrm{m}-1 /\left(1+(\mathrm{x} /(\mathrm{x}+\mathrm{y}) \mathrm{m})^{\wedge} 2\left(\mathrm{P}_{\mathrm{Y}}-1\right)-\mu 1 * \mathrm{Px}-\mu 1\right.\right. \\
& \mathrm{dP}_{\mathrm{Y}}=\mathrm{C} 1 \mathrm{n}\left(\mathrm{y} /(\mathrm{x}+\mathrm{y}) \mathrm{n}-1 /\left(1+(\mathrm{y} /(\mathrm{x}+\mathrm{y}) \mathrm{n})^{\wedge} 2(\mathrm{Px}-1)-\mu 2 * \mathrm{P}_{\mathrm{Y}}-\mu 2\right.\right.
\end{aligned}
$$


Note that our model here simplify the origin model where $\mathrm{Ci}=\mathrm{ai} / \mathrm{bi}$, with $\mathrm{bi}=1$ as the burst size of protein $\mathrm{i}, \mathrm{x} /(\mathrm{x}+\mathrm{y})=\mathrm{x} /(\mathrm{K} 2 *(\mathrm{x}+\mathrm{y}))$ as $\mathrm{k} 2=1$ is the dissociation constants standing for gene $\mathrm{x}$ binding on y's protein binding site. Regarding $\mathrm{x}$ and $\mathrm{y}$ as leading order variable, we apply phase analysis to consider the solution's stability around the three zero-energy points, which achieved through setting $\mathrm{dx}, \mathrm{dy}, \mathrm{dP} \mathrm{X}$ and $\mathrm{dP} \mathrm{Y}$ all to zero and combine the Hamiltonian's special case when $\mathrm{H}=0$ (and $\mathrm{H} \mathrm{P}=0)$ : $\mathrm{P} 1(\mathrm{x}, \mathrm{y}, \mu 2 * \mathrm{X} / \mathrm{C} 1, \mu 1 * \mathrm{Y} / \mathrm{C} 2)$, where $\mathrm{x}$ and $\mathrm{y}$ are the solution of $\mathrm{x}$ $=\mathrm{C} 1 \mu 1 *\left(1+\left(\mathrm{y} /(\mathrm{x}+\mathrm{y})^{\wedge} \mathrm{n}\right)\right.$ and $\mathrm{y}=\mathrm{C} 2 \mu 2 *(1+(\mathrm{x} /(\mathrm{x}+\mathrm{y}) \mathrm{m}), \mathrm{P} 2(\mathrm{x}, \mathrm{y}, 0,0)$, where $\mathrm{x}$ and $\mathrm{y}$ are the solution of $\mathrm{x}=\mathrm{C} 1 \mu 1\left(1+\left(\mathrm{y} /(\mathrm{x}+\mathrm{y})^{\wedge} \mathrm{n}=-\mathrm{y}=-\mathrm{C} 2 \mu 1(1+(\mathrm{x}+\mathrm{y}) \mathrm{m}\right.\right.$, and $\mathrm{P} 3(0,0,0,0)$ As P X and P Y are either zero or formula can be replaced by $\mathrm{x}$ and $\mathrm{y}$ around those three convergence points. We here, consider the analysis on $\mathrm{x}$ and $\mathrm{y}$ as following: denote $\mathrm{dx}=\mathrm{f}(\mathrm{x}, \mathrm{y})$ and $\mathrm{dy}=\mathrm{g}(\mathrm{x}, \mathrm{y})$, and the we try to find $x^{*}$ and $y^{*}$ satisfy the $f(x, y)=0$ and $g(x, y)=0$ as well as holding the zero-energy points for their momentum. Thus with approximation: $\mathrm{dx}=\mathrm{f} X(\mathrm{x} * \mathrm{y} *)(\mathrm{x}-\mathrm{x} *)+\mathrm{f} \mathrm{Y}(\mathrm{x} * \mathrm{y} *)(\mathrm{y}-\mathrm{y} *)$, and $\mathrm{dy}=\mathrm{gX}(\mathrm{x} *, \mathrm{y} *)(\mathrm{x}-\mathrm{x} *)+\mathrm{g} \mathrm{Y}(\mathrm{x} *, \mathrm{y} *)(\mathrm{y}-\mathrm{y} *)$. We have

$$
\begin{aligned}
A= & \begin{array}{ll}
f x & f y \\
g x & g y
\end{array} \\
-u 1 P x & \frac{C 2 n\left[\frac{y}{x+y}\right]^{n-1}}{\left(1+\left[\frac{y}{x+y}\right]^{n}\right)^{2}} P x \\
=-u 2 P y & \frac{C 1 m\left[\frac{y}{x+y}\right]^{m-1}}{\left(1+\left[\frac{y}{x+y}\right]^{m}\right)^{2}} P y
\end{aligned}
$$

Where there exists the $\mathrm{a}>0, \mathrm{~b}>0$ for the eigenvalue $\lambda$ :

$$
\begin{array}{rl}
\lambda \wedge 2+a & * \lambda+b=0 \\
a & =-(f x+g Y) \mid(x *, y *) \\
b & =|A|
\end{array}
$$

so that point $\left(\mathrm{x}^{*}, \mathrm{y}^{*}\right)$ is the convergence points. Thus, we discuss about the stability of the three points as following: we denote $\mathrm{X}=\left(1+\left(\mathrm{x} /(\mathrm{x}+\mathrm{y})^{\wedge} \mathrm{m}\right)\right.$ and $\mathrm{Y}=\left(1+\left(\mathrm{y} /(\mathrm{x}+\mathrm{y})^{\wedge} \mathrm{n}\right)\right.$, compute the a and $b$ as:

$$
\begin{gathered}
=\mu 1 * \operatorname{Px}+\frac{-(\mathrm{fx}+\mathrm{gY}) \mid\left(\mathrm{x} * \mathrm{~m}\left(\frac{\mathrm{x}}{\mathrm{x}+\mathrm{y}}\right)^{\mathrm{m}-1}\right.}{\left(\left(1+\frac{\mathrm{x}}{\mathrm{x}+\mathrm{y}}\right)^{\mathrm{n}}\right)^{2} \mathrm{~b}}=\mid \mathrm{A} \|(\mathrm{x} * \mathrm{y} *) \\
=\mathrm{u} 2 * \mathrm{Px} * \mathrm{PY} * \mathrm{C} 1 \mathrm{n}\left(\frac{\frac{\mathrm{y}}{(\mathrm{x}+\mathrm{y})^{\mathrm{n}-1}}}{\left(1+\frac{\mathrm{y}}{\mathrm{x}+\mathrm{y}}\right)^{\mathrm{n}}}\right)^{2} \\
\left.-\mathrm{u} 1 \mathrm{PxPYC} 1 \mathrm{~m}(\mathrm{x} /(\mathrm{x}+\mathrm{y}))^{\mathrm{a}}(\mathrm{m}-1) /(1+\mathrm{x} /(\mathrm{x}+\mathrm{y}))^{\wedge} \mathrm{n}\right)^{\wedge} 2
\end{gathered}
$$

[1] for $\mathrm{P} 1\left(\mathrm{x}, \mathrm{y}, \mu \frac{2 \mathrm{X}}{\mathrm{C} 1}, \mu 1 * \mathrm{PX} * \mathrm{PY} * \frac{\mathrm{C} 2 \mathrm{~m}\left(\frac{\mathrm{x}}{\mathrm{x}+\mathrm{y}}\right)^{\mathrm{m}-1}}{\left(1+\frac{\mathrm{x}}{\mathrm{x}+\mathrm{y}}\right)^{\mathrm{n}}}\right)^{2}$, where $\mathrm{x}$ and $\mathrm{y}$ are the solution of $\mathrm{x}=\mathrm{C} 1 / \mathrm{u} 1(1$ $+\left(y /(x+y)^{\wedge} n\right)$ and $\quad y=C 2 / / \mathrm{u} 2\left(1+\left(x /(x+y)^{\wedge} m\right)\right.$

$$
\begin{gathered}
\mathrm{a}=\mu 1 * \mathrm{u} 2 * \frac{\mathrm{x}}{\mathrm{C} 1}+\mu 1 * \mathrm{y} * \frac{\mathrm{C} 2 \mathrm{~m}\left(\frac{\mathrm{x}}{\mathrm{x}+\mathrm{y}}\right)^{\mathrm{m}-1}}{\left(\left(1+\frac{\mathrm{x}}{\mathrm{x}+\mathrm{y}}\right)^{\mathrm{n}}\right)^{2}} \\
=\mu 2^{\wedge} 2 * \mathrm{~B}^{\wedge} 2+\mu 1 * \mathrm{C} 2 * \mathrm{~A} * \mathrm{~m} *(\mathrm{x} /(\mathrm{x}+\mathrm{y}))^{\wedge}(\mathrm{m}-1) / \mu 2 * \mathrm{~A} * \mathrm{~B},
\end{gathered}
$$

[2] Thus, for $\mathrm{P} 2, \mathrm{a}=\mathrm{b}=0$. It's unstable. 
[3] for P3 $(0,0,0,0)$, same as $P 2, a=b=0$ and it's unstable.

\section{b. Two fixed points for the neuron model}

When there is no stimulus, the neuron fire spontaneously $(\mathrm{I}=0), \mathrm{w}<\beta$, the two fixed points is studied through $\mu(=\rho \sigma)$ changing with $\sigma: \mathrm{M} 0=\left(0, \varphi_{0}\right)=(0, \arcsin (-\mathrm{w} / \beta))=(0$,$0.997903)$ and $\mathrm{M} 1=(\mathrm{S} 1, \varphi 1)=\left(1, \mathrm{pi} / 2-\arcsin \left(-\mathrm{w} /\left(\beta_{-}{ }^{\rho} \mathrm{S} 0\right)\right)\right)=(1,2.568699)$.

Similar to the model a, we denote $\mathrm{f}(\mathrm{S}, \quad \varphi)=-S+\sigma\left(\cos \varphi-\cos \varphi_{0}\right)+I, \mathrm{~g}(\mathrm{~S}, \quad \varphi)$ $=w+(\beta-\rho S) \sin \varphi$ and the stability of M0 is linear thus can be analysed with the Jacobian of the coupled (**) at $\varphi_{0}$ :

$A=\left(\begin{array}{ll}f s & f \varphi \\ g s & g \varphi\end{array}\right)=\left(\begin{array}{lr}-1 & -\sigma \sin \left(\varphi_{0}\right) \\ -\rho \sin \left(\varphi_{0}\right) & \beta \sin \left(\varphi_{0}\right)\end{array}\right)=\left(\begin{array}{cc}-1 & \frac{\sigma}{1.19} \\ \frac{1}{1.19} & -\frac{1}{1.4161}\end{array}\right)$

we try to find $\mathrm{S}^{*}$ and $\varphi^{*}$ satisfy the $\mathrm{f}\left(\mathrm{S}^{*}, \varphi^{*}\right)=0$ and $\mathrm{g}\left(\mathrm{S}^{*}, \varphi^{*}\right)=0$ as well as holding the zeroenergy points for their momentum. Thus with approximation: $d S=f s(S *, \varphi *)(S-S *)+f \varphi(S *, \varphi$ $*)(\varphi-\varphi *)$, and $\mathrm{d} \varphi=\mathrm{gs}(\mathrm{S} *, \varphi *)(\mathrm{S}-\mathrm{S} *)+\mathrm{g} \varphi(\mathrm{S} *, \varphi *)(\varphi-\varphi *)$. Where there exists the $\mathrm{a}>0, \mathrm{~b}>0$ for the eigenvalue $\lambda: \lambda^{\wedge} 2+\mathrm{a} * \lambda+\mathrm{b}=0$. Thus, $\mathrm{a}=-(\mathrm{fs}+\mathrm{g} \varphi) \mid\left(\mathrm{S}^{*}, \varphi^{*}\right)=-1+\beta \sin (\varphi *)=-2, \mathrm{~b}=$ $|\mathrm{A}|=-\left(\beta \sin (\varphi *)+\sigma \sin \left(\varphi_{*}\right) * \rho \sin (\varphi *)\right)=-\left(-\mathrm{w}+\mu \mathrm{c}(\mathrm{w} / \beta)^{\wedge} 2\right)=-0.323219$, leading to $\lambda=$ $=0.5 *\left(\eta-1 \pm \sqrt{(\eta-1)^{2}+4\left(\rho \sigma \sin \left(\varphi_{0}\right)^{2}+\eta\right)}\right)=\{0.817465,-1.361948\}, \mu \mathrm{c}$ $=0.95839$

[1] M0 is stable fixed-point for $\mu<\mu \mathrm{c}, \mathrm{M} 1$ is unstable with $\varphi 1>\varphi 0$ and $\mathrm{S} 1>\mathrm{S} 0$

[2] M0 is attractor for $\mu=\mu \mathrm{c}$, another fixed point $\mathrm{M} 1=\mathrm{M} 0$

[3] M0 is unstable fixed-point for $\mu>\mu \mathrm{c}, \mathrm{M} 1$ is stable with $\varphi 1>\varphi 0$ and $\mathrm{S} 1<\mathrm{S} 0$

On quantum field, short introduction about two Rydberg here is covered for comparison.

[1] $\mathrm{u}<<\Omega_{j}$ makes it unnecessary to address two atoms separately. Because $\Omega_{1}=\Omega_{2}=\Omega$, we can set $\mathrm{d} 1=\mathrm{d} 2=0$ and realize the gate with a)drive a pi-pulse to two atoms->b)pause for $\mathrm{dt}=\varphi / \mathrm{u}$ $>c)$ drive a pi-pulse again to two atoms. Note that the accumulated phase is sensitive to atomic distance and the decay probability is approximately $\mathrm{pl}=2 \varphi \mathrm{Y} / \mathrm{u}$

[2]Adiabatical condition for $\mathrm{u} \gg>\Omega_{j}$ still makes it unnecessary to address two atoms separately. As $\Omega_{1}=\Omega_{2}=\Omega$, d1 $=\mathrm{d} 2=0$ naturally hold due to the slow on time scale given by the two parameter although larger than trap oscillation frequrncy. The detuning includes a Stark shift as connected to initial state $|\mathrm{gg}\rangle$ with energy $\mathrm{e}_{\mathrm{gg}}(\mathrm{t})=\operatorname{sgn}(d)\left(|d|-\left(d^{\wedge} 2+2 \Omega \wedge 2\right)^{\wedge} 1 / 2\right) / 2$ driven adiabatically, where $d=\mathrm{d}-\Omega \wedge 2 /(4 \mathrm{~d}+2 \mathrm{u})$ while $|\mathrm{eg}\rangle$ and $|\mathrm{ge}\rangle$ are achieved with $\mathrm{e}_{\mathrm{eg}}(\mathrm{t})=$

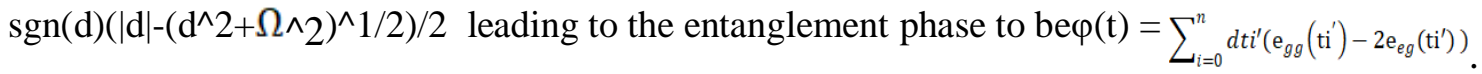
[3] $\mathrm{u}<<\Omega_{j}$ generally requires the atoms to be set differently at $\Omega_{1}, \Omega_{2}$ differently. But we can still set $d j=0$ with the cost of sign change in the wave function through a)drive a pi-pulse to the first atom->b)a 2pi-pulse to the second c)drive a pi-pulse again to the first atom. 


\subsection{Dynamics Analysis}

\section{a. HMC dynamics for protein and mRNA}
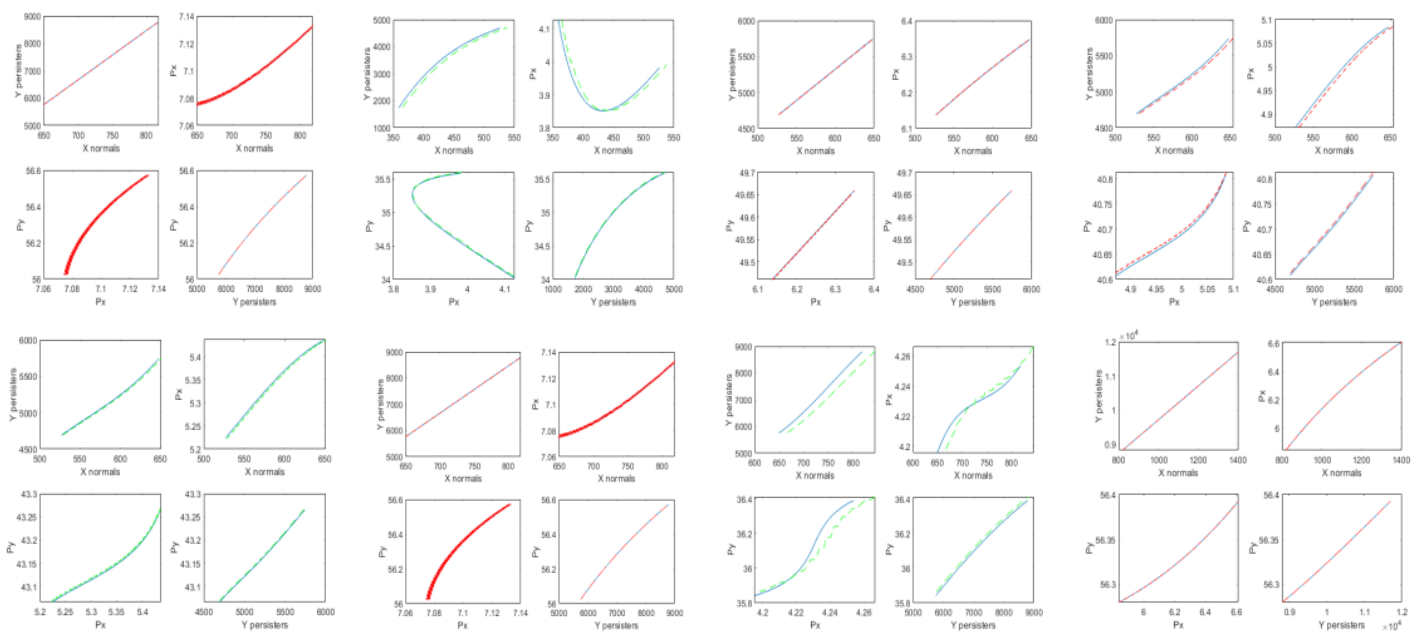

Figure 3(a)-(h) transition between mRNA and protein

In the second part here, with regard to the detailed behavior of mRNA and protein dnamics, we look into their momentum and numbers with 6 status (only the firs 2(a)-2(c) and the last 2(d)-2(f) transition xamples of the origin 3groups*5transition statuses figures) in all are studied detailedly while the whole data based on 15 status. As we only investigated the positive direction, the red ones (top left) the application on clinical data while green one (top right) in the larger scaled simulation with more transition status (blue dashed line is the predicted dynamics)..Note that the persisters and normals are the roles they take in the whole process(considering from bifurcation to catastrophe and extinction) where here they can be all considered as promoters as their numbers both grows in this process until the last status as their interaction in constant environment is of our main interest as we mentioned before. Generally, with small change studied in one status, the trend is more significant than the larger scale transition. For instance, the green simulation are always more sensitive to the momentum change and shows them more significantly on the cell trajectory comparing to the red clinical transition (we manually break one clinical status into sub-status in simulations. )

Specifically, in the 1-> 2 transition, the production of the HS 1 is slightly faster than hax 1 with the accelerate from faster to slower as well as the hax $10 \mathrm{~s}$ momentum decreases from fast to slow while HS $01 \mathrm{~s}$ momentum increases from fast to slow similarly. The larger scaled simulation show the trend similarly but with larger momentum difference and thus gives out the curve trajectory instead of straight line in the top left figure; On contrary, in 2->3 transition, both the clinical application and larger simulation give totally the same behavior according to the dynamics, where proteins products faster than mRNA but with similar acceleration. Other transition can be similarly analysis. Note that from the $4->5$ of the larger scale simulation, there starts to show the switching where the protein changes into persisters with degradation instead of production which can be both detected from cell numbers figure in the top left and momentum figures in the right bottom although the fewer status contained clinical data does not show this behavior yet. In the last transition status, the switching of proteins becoming into persister is detected in both clinical process and simulation, where in the clinical data, the momentum change of roteins and mRNA are both linear process while in simulation, the momentum of the mRNA grows slightly from faster to slower and proteins degradate slightly from faster to slower as well 
and in the last short time, proteins go back to normals again which according to the rising number change in the top left and increase in the momentum both relatively to mRNA(left bottom) and absolutely (right bottom.) )

In the second series of figures2(c),2(f), we compute the mean time to switch approximation with the solution based on mapping to their difference space where we choose the object as 1) sigle population of mRNA to the end of the transition(top left);2) sigle population of proteins to the end of the transition (top right); 3) mRNA population to the end status of protein(left bottom) and 4) proteins population to the end status of mRNA.(right bottom.)There gives some different patterns, as in the 1->2, both the mRNA and proteins has the mean time to switch increase linearly with their number change while there exists one significatly longer time at 0.4 for the proteins compare to the final status of mRNA and one totally unstable transition recorded; In the $2->3$, all the MTS increase linearly with the cell number growth; In the 4-> 5, as there exhists the decrease of proteins thus there exhists one negative MTS stands for the status; And in 5->6, the last status for the proteins again, compared to the final status where the number back to increase, the previous degradation status also leads to the minus MTS but positive to the mRNA as they both grow in the end. In the last part, Further application using the transition matrix of the model, we compute some basic markov chain quantities based on the stochastic process as following with the pre-computation result(in availability):

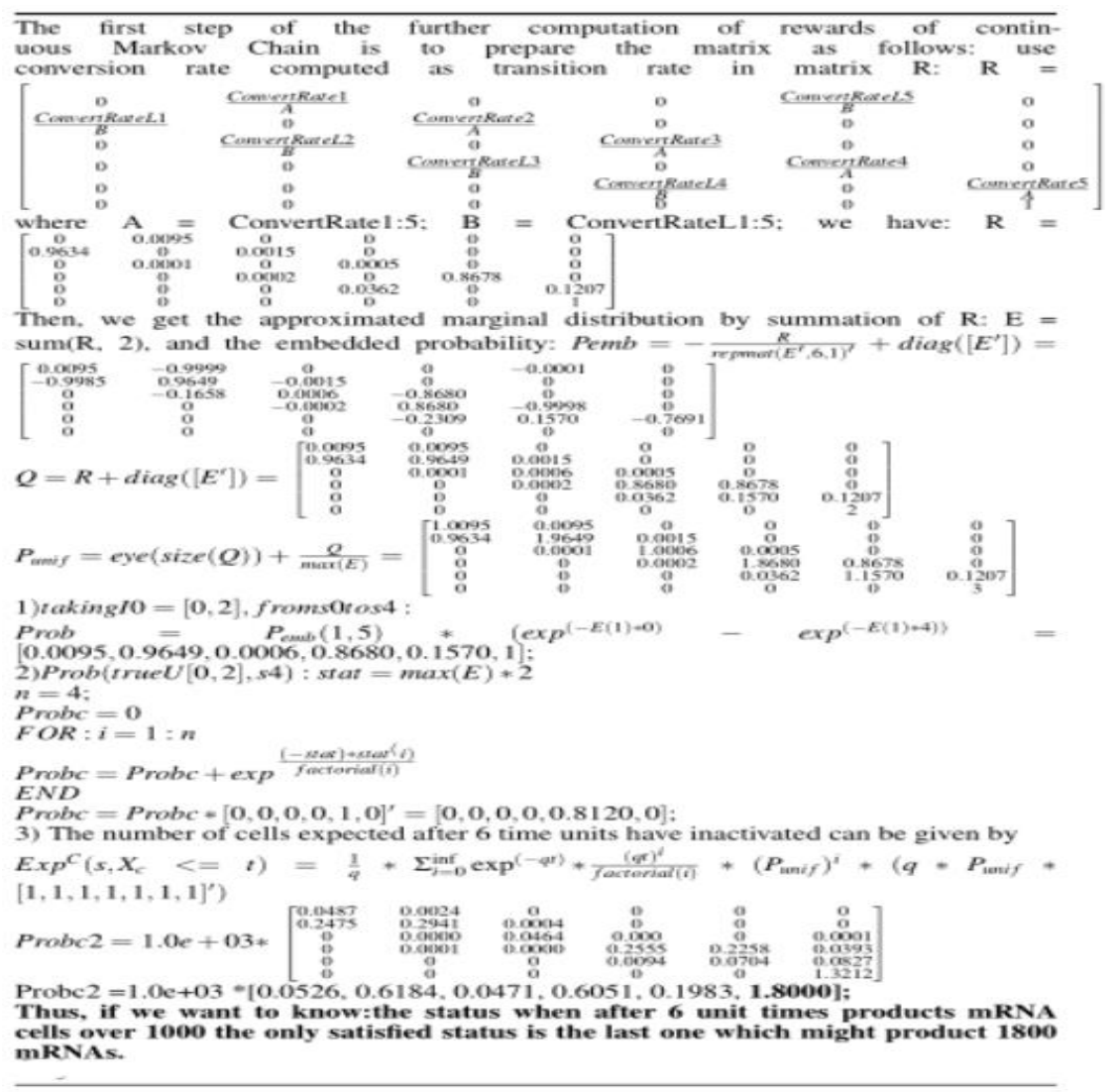

Figure 4 computation of probabilistic safety

In the probabilistic safety computation by finite DTMC abstraction, the computation is based on the differential cognitive $(*)$ in 2.3.3 with the previous unified matrix of CTMC composed of 6 transition status and 6 time points for each, $\mathrm{dX}=\mathrm{P}(:, 2: 6)=\mathrm{P}(:, 1: 5)$; 
Continue the error bounds for time discretization in 2.3.3, considering mean: $\mathrm{Mu}=\operatorname{mean}(\mathrm{P}, 1)$;

Sigma $2=\operatorname{var}(\mathrm{P}, 1) ; \operatorname{Sigma}=$

$\operatorname{std}(\mathrm{P}, 1) ; \mathrm{W} \sim \mathrm{N}(\mathrm{Mu}, \operatorname{Sigma} 2)=\operatorname{repmat}($ ones $(1,6) . / \sqrt{ } 2 *$ pi*Sigma2),6,1). $* \exp (-\mathrm{P}-$

$\operatorname{repmat}(\mathrm{Mu}, 6,1)) . \wedge 2 / 2 . /$ repmat(Sigma.^2,6,1); Then the Brownian, $\mathrm{G}=\exp \left(\mathrm{Mu} . *^{*}-\right.$

Sigma. ${ }^{\wedge} \cdot{ }^{*} \mathrm{t} / 2+$ Sigma. $\left.* \mathrm{~W}\right)$;

With $d=\sqrt{ }\left((X)^{\wedge} 2\right)$,

As $\mathrm{dt}=1$ fixed, $\mathrm{Kd}=\max (\mathrm{d})=1.3433$; And with sampling time $\mathrm{h}=\min (2(-6) / 2 / \sqrt{ }(2) / \mathrm{K} 2 / \mathrm{Kd}, 2(-$

6)) $=0.28558$

Finally, with $\mathrm{I}=0: 5, \mathrm{Pds}=\mathrm{T}, \mathrm{z} 0=1$, we can achieve:

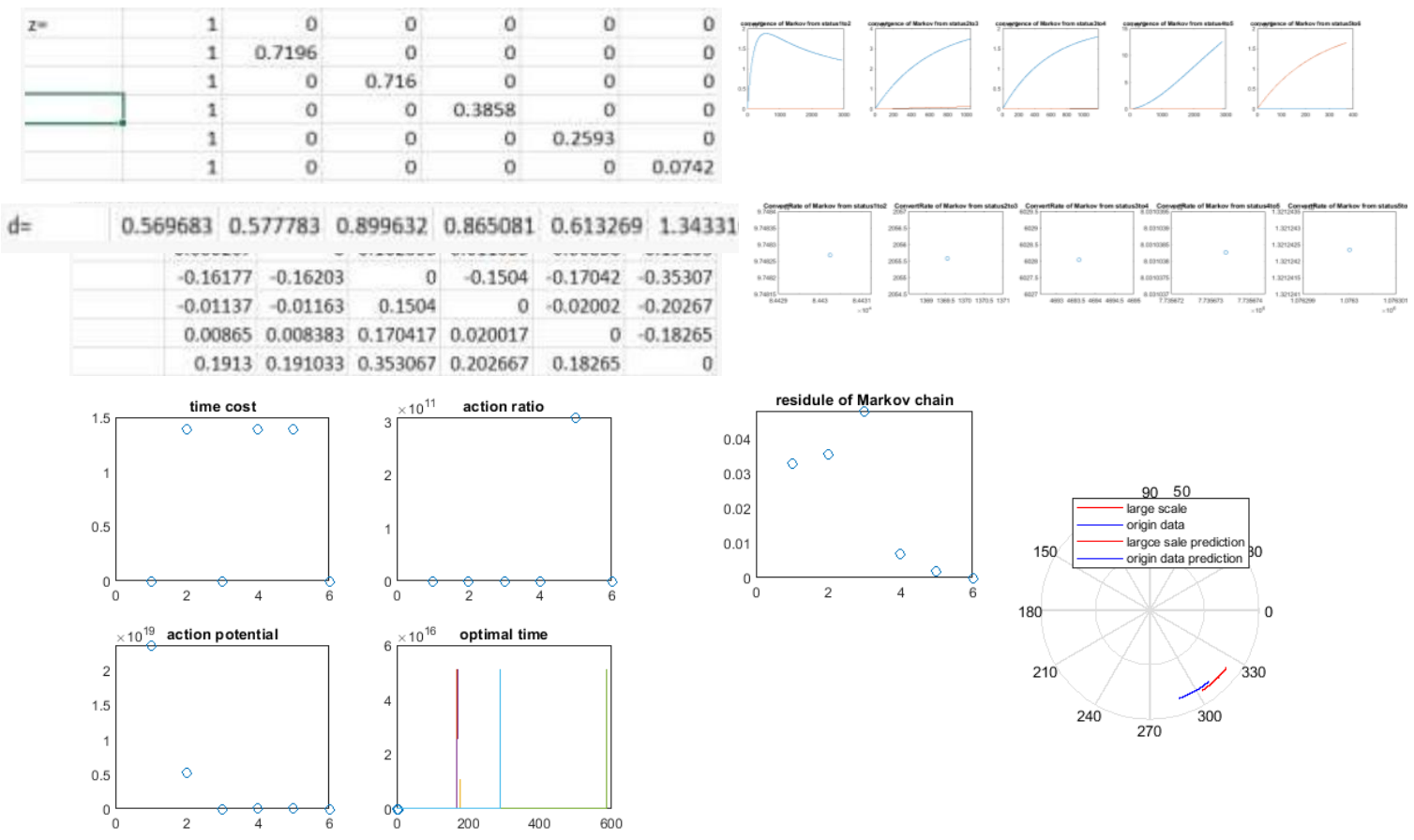

Figure 5. (i) convergence of MC; (j)convert rate from low I to $\mathrm{i}+1$ level $(\mathrm{k})$ total metrics related to time domain; (k) residule of Markov Chain and phase.

\section{b. Application on neural network oscillation model analogue to computation on quantum computer with all versus-nothing arguments}

Specifically, we apply the model on neurons. Considering the energy computation, the flow of ions crosses the membrane, associating the oscillations and construct the up-states and downstates with the interactions in the network. Although coupling of neurons are specific to event/task, to simplify, we adopt the Hopfield network instead (the weight connecting two states is symmetry: $\mathrm{w} 1=\mathrm{w} 2=\mathrm{w}$ ).

Classically, we start with one unit, $\mathrm{Vi}=\mathrm{V}, \mathrm{C} \_\mathrm{Mg}=1$, steady state $\left(\mathrm{V}_{\mathrm{E}}=0\right) \mathrm{I}-\mathrm{V}$ curve can be drawn according to $\mathrm{I}=\mathrm{g}_{\mathrm{GABAI}, \mathrm{E}}\left(\mathrm{V}-\mathrm{V}_{\mathrm{GABA}}\right)+\mathrm{g}_{\mathrm{AMPAI}, \mathrm{E}} \mathrm{V}+\mathrm{g}_{\mathrm{NMDAI}, \mathrm{E}} \mathrm{V} /\left(1+1 \mathrm{e}^{-.0 .062 * \mathrm{~V} / 3.57}\right)$, assuming staring from inhibitory population, the synaptic reversal potentials of excitatory being 0 . According to the experiment data of synaptic conductance $\mathrm{g}$ between pyramidal and interneuron population[], with the parameters in TPN: $\mathrm{g}_{\mathrm{GABAE}}=0.0006681, \mathrm{~g}_{\mathrm{GABAI}}=0.0005120, \mathrm{~g}_{\mathrm{AMPAE}}=0.0001905, \mathrm{~g}_{\mathrm{AMPAI}}=$ $0.0001460, \quad \mathrm{~g}_{\mathrm{NMDAE}}=\mathrm{k} 5 / \mathrm{NI} * 0.001, \quad \mathrm{~g}_{\mathrm{NMDA}}=60 / \mathrm{NI} * 0.001, \quad \mathrm{k} 5=$ $[6.15,7.75,10.25,11.25,12.65,13.25], \mathrm{NI}=4096 ;$ in $\mathrm{TNN}: \mathrm{g}_{\mathrm{GABAE}}=0.0006681 * 1.5, \mathrm{~g}_{\mathrm{GABAI}}=$ 
$0.000512 * 1.5, \mathrm{~g}_{\mathrm{AMPAE}}=0.0001905 * 4, \mathrm{~g}_{\mathrm{AMPAI}}=0.0001460 * 4, \mathrm{~g}_{\mathrm{NMDAE}}=60 / \mathrm{NI} * 0.001, \mathrm{~g}_{\mathrm{NMDAI}}=$ $\mathrm{k} 5 / \mathrm{NI} * 0.001, \mathrm{k} 5=[6.15,7.75,10.25,11.25,12.65,13.25], \mathrm{NI}=4096$

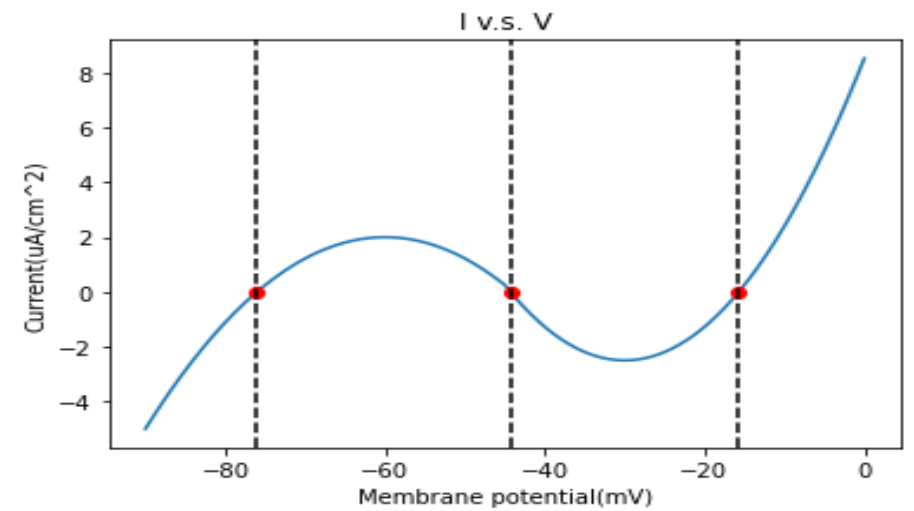

Quantomechanical, the problem of finding stable state can be discussed on kinematic phase:

In a $3 \mathrm{D}$ potential $V^{\beta}(\mathbf{x})=v^{\beta}(x)+v_{\perp}(y)+v_{\perp}(z)$,

where $\mathrm{v}$ are single-wells and $v^{a}, v^{b}$ are centered around $\mathrm{x} 0$ and 0 individually so that the atom has its state $\mid \Psi+(\mathrm{x})>$ composed by $\psi+, \psi \perp$ (ground-state wave functions of $v^{a}$ with eigenvalue $\mathrm{Ea}$ and $\mathrm{v} \perp$ with eigenvalue $\mathrm{E} \perp$ ):

$$
\left\langle\mathbf{x} \mid \Psi_{+}\right\rangle \equiv \Psi_{+}(\mathbf{x})=\psi_{+}(x) \psi_{\perp}(y) \psi_{\perp}(z)(* * *)
$$

And $\Psi+(\mathrm{x})$ is peaked at the stable point $\mathrm{x} 0=(\mathrm{x} 0,0,0)$, coinsiding with the center of $V^{a}(\mathrm{x})$ which can be occupied by the $V^{b}(\mathrm{x})$. That is in internal state $|\mathrm{a}\rangle$, the atom has the motional state which after time $\mathrm{t}$ still unchanged up to its phase $\varphi^{a}$ being $(\mathrm{Ea}+2 \mathrm{E} \perp) \mathrm{t} / \mathrm{h}$ while in state $|\mathrm{b}\rangle$, sue to the kinematical evolution of $\varphi^{b}$, it will come back to the initial position after oscillating.

If we consider two atoms 1 and 2 both initialized at $\mathrm{t}=0$ with $|\Psi+(\mathrm{x})\rangle$ and $\Psi-(\mathrm{x})\rangle$ same as in $(* * *)$ with $\psi^{-}(\mathrm{x}) \equiv \psi^{+}(-\mathrm{x})$. Similar as firing rate model $\mathrm{R}[\mathrm{I}]$, the particles are subject to step function $\mathrm{S}(\mathrm{xi})$ related potentials $\sum_{c=\{+,-\}} S(c x i) V^{a, b}$ (cxi). The reduced form as 1D two particle Schrodinger can be achieved with integrating the variables:

$\mathcal{H}^{\beta_{1} \beta_{2}}=\sum_{i=1}^{2}\left[\frac{\left(p_{i}\right)^{2}}{2 m}+w^{\beta_{i}}\left(x_{i}, t\right)\right]+u_{x}^{\beta_{1} \beta_{2}}\left(x_{1}-x_{2}\right)$

And with setting $\beta 1=\beta 2 \equiv \beta$, the symmetric under particle interchange :

$$
\left|\psi^{\beta \beta}(0)\right\rangle \approx \frac{\left|\psi_{+}\right\rangle_{1}\left|\psi_{-}\right\rangle_{2}+\left|\psi_{-}\right\rangle_{1}\left|\psi_{+}\right\rangle_{2}}{\sqrt{2}} \otimes|\beta\rangle_{1} \otimes|\beta\rangle_{2} \text {, where }\langle\psi-| \psi+>\ll 1 \text { has been neglected. }
$$

This gives out same result that if both atoms are in state $|a\rangle$, then due to the collisional phase $\varphi^{a a}=0$, no interaction takes place. 


\section{CONClusion}

In general, Hamiltonian markov chain advantage over the markov chain random walk with its faster convergence. As in 2(i) and 2(j), the convergence(variation to mean) of the markov chain hamilton is in blue line and the red line for clinical data and simulation on more possible transition status, giving different convergence but similar phase interval(according to $2(\mathrm{k})$ ), interestingly. The last status transition converge the worst followed by the fist transition. And the result simulated with more markov chain status converges better than the clinical results. And according to the convert rate, the mRNA to Protein transfer ratio should be the highest when starting, and goes especially lower in the last two status which is in assistance to the protein binding as we cut off the process around the convergence point where the two population has reached metastability. According to the simulation result, the protein has gone through the switching process changing from normals to persisters and back to normal (bursts in optimal time in 2(j) might also due to the switch.).

Mean while, as the second population providing food(protein) to the other's binding site and eithr activiate or deactivate it, it works as the extrinsic noise induced the excitability or exhibition of the other gene. Here, as we choose hax 1 and HS 1, they work as promoters for each others. One noticable computation is the reward computaiton based on stochastic model selection which is useful in predict the possible status of the cell numbers easily with precomputation. And we can consider correct the transition matrix with simualted clinical tested results to improve the prediction as well. On the other hand, the most important calculation action potential is easier to be achieved through Hamilton as we proved with geometric minimum action and stochastic approximation. Ohter methods can cover Hamilton Jacobian matrix, WKB and etc. As we also improve the algorithm with adding hierarchical markov in calculating number of cells in different status only record successful move according to the tolerance based on action potential and residual of prediction numbers both, the convergence of the algorithm is guaranteed. And further research can be conducted on the whole process from bifurcation to catastrophe and extinction as well. Problem with multi population is also possible. As hax 1 is observed to have function in signaling and regulating of genes especially in learning systems and motor related brain function, this switching model study related to its binding might help to predict the cell numbers and production or degradation rate especially later with further study into both with promoters and persisters as to test different drug and their efficiency on the aging process related disease.

In the computation of reachability, the approximation with DTMC mainly compute the kernels of Brownian with shift, finally discretize the original switching diffusion process. As the DTMC gives out the kernel with probability instead of the ratio, it is then convenient to be written into transition matrix $\mathrm{Pdx}$ which is discretized from $\mathrm{Sdx}$ on finite space state and gives out the reachability with error $\mathrm{I} / \mathrm{h} *\left(\mathrm{Kdx}+\exp \left(-2^{\wedge} \mathrm{n}-2^{\wedge} \mathrm{n} / 2+1\right)\right)$.

As the proof in Appendix, the error bounds with Lipschitz constants converged with prominent $\mathrm{K}=\mathrm{mh} 1+\mathrm{Lh} 2$. And the computed result $\mathrm{N}^{*} \mathrm{~K} * \mathrm{dx}$ is here is 0.453 with $\mathrm{N}=6, \mathrm{~m}=\mathrm{L}=2, \mathrm{dx}=0.002$ and $\mathrm{h} 1=0.001$, and $\mathrm{h} 2=\operatorname{ceil}(\mathrm{h} 2 * \mathrm{~N})=1.71$.

Since the final result of the continuous process is not of probability range thus we normalize it with $\mathrm{P}=$ ratio/sum(ratio) and the DTMC approximation shown in the figure is the approachability(1-safety.) The result of the test tested continuous embedded matrix and $\mathrm{h}=0, \mathrm{p}$ $=0.0515$, ci $=-0.7212,0.0029$, stats = struct with stat: $-2.2103(\mathrm{df}: 10)$, do not reject the hypothesis that the two process. As the final safety consistently for two methods gives highest concentration for the last state showing the example computation's direction from off to on, although there is the slight difference that the forth in the continuous process is relative lower comparing to its other five states as well as the one in the discrete process states. The DTMC 
gives strictly increasing concentration from off to on during the 6 states. switching diffusion is a commonly used model in genetic field, not only useful in the transmission of different molecules but also can be derived into analytical models giving straight transfer information about some process with either concentration change or energy change.

About the neuron switch model, it is clear that there is similarity between classical computation and quantum computation with regards to the phase and excitation. This is not surprising since the process identify the excitation is to compare the spectrum with threshold which basically counting on the transform from time domain to frequency domain and such a process can be usually realized with operation on exponent computation while the quantum mechanics is origin from the computation of quantized energy which is also with exponent bases, for instance the most well known poisson distribution and normal distribution as well as the transform as FFT, the same level of computation at the first place correlate these two. And with our experiment, the transform can be illustrated with phase initialized at specific state clearly, thus the entanglement is expressed in quantum computation as well as the classical computation in stability analysis.

As mentioned in the introduction, the similarity exists in the stimulus at hippocampus so as to simulate spontaneous neuron activities and conducting pi-pulse on atoms with different orders so as to excite it to higher states, this is still not quantitively studied in this paper although some related phase operations are covered, as of both theoretical and experimental value, worth being explored more in the future. Many properties of quantum computation with regards to semantics is also of large usage and since neural network is applied frequently in this field, similar application on quantum computation is also a future direction.

\section{ACKNOWLEDGEMENTS}

Although I have graduated with excellence graduates awards, I am still working in the university currently cooperated with university neural dynamic lab. Thanks to all the supervisors and colleagues I have worked with.

\section{REFERENCES}

[1] Freedman,H.I.,Dynamics of simple gene-network motifs subject to extrinsic fluctua-tions, 1980

[2] Noufe H. Aljahdaly,Analytical Solutions of a Modified Predator-Prey Model through a New Ecological Interaction,2019 ensembl,https://www.ensembl.org/index.html

[3] Ingo Lohmar, Baruch Meerson, Switching between phenotypes and population extinction, Racah Institute of Physics, 2018

[4] Daniel Durstewitz1, Jeremy K. Seamans1 and Terrence JRu. Sejnowski1,2, Neurocomputational models of working memory, 2000

[5] MARCELO CAMPERI, XIAO-JING WANG, A Model of Visuospatial Working Memory in Prefrontal Cortex: Recurrent Network and Cellular Bistability, 1998

[6] Marta Kwiatkowdka, Gethin Norman, David Parker, Stochastic Model Checking, Oxford, 2019

[7] David Martínez-Rubio, Varun Kanade, Patrick Rebeschini, Decentralized Cooperative Stochastic Bandits, Oxford, 2005 Paul C Bressloff, Stochastic swiching in biology: from genotype to phenotype, Department of Mathematics, University of Utah, 2017 Baier, Christel, Principles of Model checking, Massachusetts Instititute of Technology,2008

[8] Zhu Z, Wang R and Zhu F (2018) The Energy Coding of a Structural Neural Network Based on the Hodgkin-Huxley Model. Front. Neurosci. 12:122. doi: 10.3389/fnins.2018.00122

[9] David Colliaux, Colin Molter, Yoko Yamaguchi, Working memory dynamics and spontaneous activity in a flip-flop oscillations network model with a Milnor attractor, 2009

[10] D. Jaksch, J.I. Cirac, and P. Zoller, S.L. Rolston, R. Cote and M.D. Lukin , Fast quantum gates for neutral atoms, 2000, arXiv:quant-ph/0004038v2, DOI:10.1103/PhysRevLett.85.2208

[11] Samson Abramsky, Lucien Hardy, Logical Bell Inequalities,2012, arXiv:1203.1352v4 
[12] Cheng Lv, Xiaoguang Li, Fangting Li, Tiejun Li, Constructing the Energy Landscape for Genetic Switching System Driven by Intrinsic Noise, Peking University, 2014

[13] C.W. Gardiner, Handbook of Stochastic Methods for Physics, Chemistry and the Natural Sciences, 4th ed, Handbook of Stochastic Methods for Physics, Chemistry and the Natural Sciences, 4th ed.,Springer, 2009 Alberto Finzi, Thomas Lukasiewicz, Game-Theoretic Agent Programming in GologLuca Cardelli, Marta Kwiatkowowska, Robustness Guarantee.Yasar Demirel, in Nonequalibrium Thermodynamics (Third Edition), 2014

[14] Matthias Heymann, Eric Vanden-Eijnden, Geometric Minimum Action Metod: A Least Action Principle on the Space of Curves, Courant Institute, 2007

[15] Approximate Model Checking of Stochastic Hybrid Systems: Alessandro Abate, Joost-Pieter Katoen, John Lygeros, Maria Prandini. Marta Kwiatkowska, Gethin Norman, Stochastics Model Checking

[16] Author Robert Balson Dingle, Their Derivation and Interpretation, Academic Press, 1973 J.C., Gittins, Keble College, Oxford, 2010

[17] Samson Abramsky1, Rui Soares Barbosa1, Giovanni Carù1 and Simon Perdrix, A complete characterization of all-versus-nothing arguments for stabilizer states, 2017, http://dx.doi.org/10.1098/rsta.2016.0385

[18] Zahid Ur Rehman, Quorum-Quenching Bacteria Isolated From Red Sea Sediments Reduce Biofilm Formation, KAUST,2016

[19] An experimental investigation on the health monitoring of concrete structures using piezoelectric transducers at various environmental temperatures.

[20] Ali, Isra, Alfarouk, Khalid O., Reshkin, Stephan J., Ibrahium, Muntaser E., Doxycy-cline as Potential Anti-cancer Agent, Anti Cancer Agents, 2018

[21] Xianjun Cheng, Yue Yuan, Yihong Wang, Rubin Wang, Neural antagonistic mechanism between default-mode and task-positive networks, 2020, DOI: https://doi.org/10.1016/j.neucom.2020.07.079

[22] G.S. Skone, Irina Voiculescu, Stratagems for effective function evaluation in computa-tional chemistry, Oxford, 2010

[23] Peter Minary, Charlotte M Deane, Explorong peptide/MHC detachment process using hiearchical natural move Monte Carlo, Oxford, 2015Biancalani, E. Giamperi, A. Bazzani, G. Catellani, and A. M aritan, Phys.Soc, Jpn., Physics, 2015

[24] D.M. Roma, R.A. P'Flanagan, A.E. Ruckenstein, A.M. Sengupta, R., Optimal path to epigenetic switching, Rarah Institute of Physics,2015 C HASTE : incorporating a novel multi-scale spatial and temporal algorithm into a llarge-scale open source librarty B Y M IGUEL O. B ERNABEU 1 , R AFEL B ORDAS 1 , P RAS P ATHMANATHAN 1 ,J OE P ITT -F RANCIS 1 , J ONATHAN C OOPER 1 , A LAN G ARNY 2 , D AVIDJ. G AVAGHAN 1, B LANCA R ODRIGUEZ 1 , J AMES A. S OUTHERN AND J ONATHAN P. W HITELEY

[25] Chris Holmes Leonhard Held Bayesian Ausiliary Variable Models for Binary and Multinomiak Regression

[26] Michael Betancourt and Mark Girolami Hamiltonian Monte Carlo for Hierarchical Model

[27] rffany Amariuta, Ynag luo, Steven Gazal, Emma E. Davenport, Bryce van de Geijn, Kazuyoshi Ishigaki, Alkes L. Price, IMPACT: Genomic Annotation of Cell-State-Specific Regulatory Elements Inferred from the Epigenome of Bound Transcription Factors.

[28] Dynamics statistic Markov chain, Alessandro Abate, Joost-Pieter Katoen, Alexandru Mereacrem 


\section{AuTHORS}

Qin He, Received master degree in data engineering and machine learning with physics minor. During Bachelor (Maths bachelor in ECUST, student union technology and entrepreneur department academic division leader, minor in English), did research study in dynamics neuron networks lab, using statistics, data mining basic skills. Minor in English. In currently master program, doing neuroscience research study related to neuro imaging, neuron computation, minoring in Physics.) Main field in Mathematics and programming, with equal interest in other natural science and technology. And due to past background in machine learning with disciplinary projects, especially related to biomedical and neuroscience in processing time series, frequency spectrums and Bayes models combining stochastic process, computational modeling with python, matlab, $\mathrm{C} / \mathrm{C}++$ and a little bit $\mathrm{C \#}$.

Rubin Wang, Received the Ph.D. degree in electronic and mechanical engineering from Nagoya University, Nagoya, Japan, in 1998., He is currently one of the Director of the Institute of Cognitive Neurodynamics, East China University of Science and Technology, Shanghai, China. His research interests are in the areas of cognitive neurodynamics, coding and decoding theory in brain information processing, complexity theory, analysis of biological neural networks, and computational vision and audition., Dr. Wang serves as the founding Editor-in-Chief of Cognitive Neurodynamics.

Xiaochuan Pan, Received the Ph.D. degree in biophysics, University of Chinese Academy of Sciences, in 1997.,He is currently one of the Director of the Institute of Cognitive Neurodynamics, East China University of Science and Technology, Shanghai, China. His research interests are in the areas of cognitive neurodynamics, coding and decoding theory in brain information processing, complexity theory, analysis of biological neural networks, and computational vision and audition. Dt.Pan is professional at behaviour models related to deduction, conditional random dynamics and optimization.

\section{APPENDIX}

Because of paper limit see:

https://github.com/LilyHeAsamiko/QC/blob/master/UAE_ADCO(2021.02.21_02.17)/appendix\%20of\%20

Genetic $\% 20$ Switches $\% 20$ between $\% 20$ two $\% 20$ population $\% 20$ with $\% 20$ regards $\% 20$ to $\% 20 \mathrm{mRNA} \% 20$ and $\%$

20proteins\%20applying\%20Markov\%20Chain\%20Stochastic\%20Model\%20Check_cor.docx

(C) 2021 By AIRCC Publishing Corporation. This article is published under the Creative Commons Attribution (CC BY) license. 
AUTHOR INDEX

Hikaru Morita

01

Ismailova Aisulu

17

Juan Zhao

35,53

Qin He

71

Rubin Wang

71

Saeko Matsuura

01

Shopagulov Olzhas

17

Tretyakov Igor

17

Xiaochuan Pan

71

Zhitang Li

35,53 